\title{
Extragalactic Globular Clusters and Galaxy Formation
}

\author{
JEAN P. BRodie AND JAY StRADER \\ UCO/Lick Observatory
}

Key Words globular clusters, galaxy formation, stellar populations

\begin{abstract}
Globular cluster (GC) systems have now been studied in galaxies ranging from dwarfs to giants and spanning the full Hubble sequence of morphological types. Imaging and spectroscopy with the Hubble Space Telescope and large ground-based telescopes have together established that most galaxies have bimodal color distributions that reflect two subpopulations of old GCs: metalpoor and metal-rich. The characteristics of both subpopulations are correlated with those of their parent galaxies. We argue that metal-poor GCs formed in low-mass dark matter halos in the early universe and that their properties reflect biased galaxy assembly. The metal-rich GCs were born in the subsequent dissipational buildup of their parent galaxies and their ages and abundances indicate that most massive early-type galaxies formed the bulk of their stars at early times. Detailed studies of both subpopulations offer some of the strongest constraints on hierarchical galaxy formation that can be obtained in the near-field.
\end{abstract}

\section{CONTENTS}

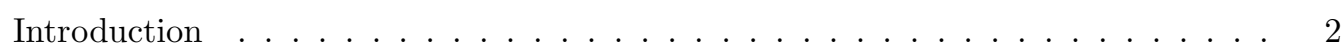

Color Bimodalitv: Globular Cluster Subpopulations . . . . . . . . . . . . . . . . . 3

Scenarios for Bimodalit . . . . . . . . . . . . . . . . . . . . . . . 8

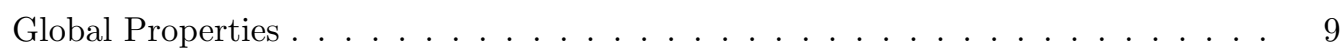

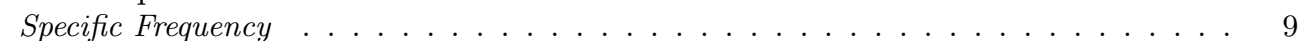

Radial and Azimuthal Distributions . . . . . . . . . . . . . . . . . . . . . . . . . . . 12

Variations with Galaxy Morpholoqu . . . . . . . . . . . . . . . . . . . . . . . . . . . 13

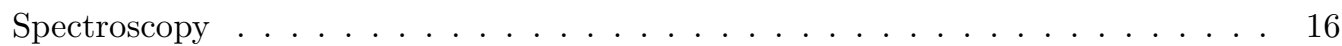

Metallicities and Ages ........................... 17

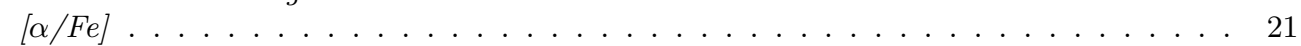

Abundance Anomalies . . . . . . . . . . . . . . . . . . 23

Near-IR Imaging . . . . . . . . . . . . . . . . . . . . . . . . . . . . . . . . . . . . . 23

Globular Cluster-Field Star Connections . . . . . . . . . . . . . . . . . . 25

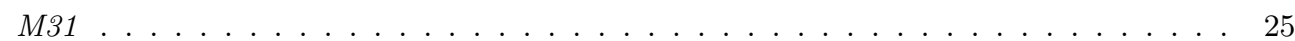

NGC5128. . . . . . . . . . . . . . . . . . . . . . . . . . . . 28

Other Galaxies . . . . . . . . . . . . . . . . . . . . . 30

Kinematics ........................... . . . . . . . . . . . . . . 


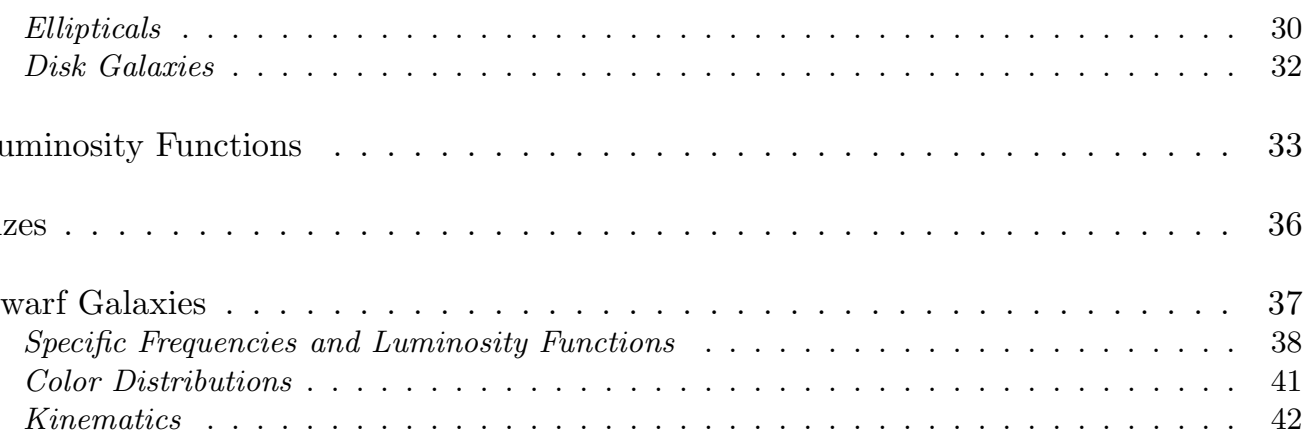

Globular Cluster Formation . . . . . . . . . . . . . . . . . . . . . . . . . . . 42

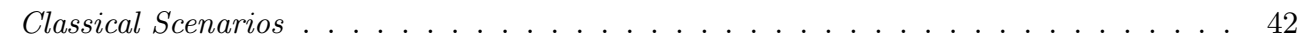

Hierarchical Merqinq and Biasinq: Recent Scenarios . . . . . . . . . . . . . . . . . 44

Cosmological Formation of Metal-poor Globular Clusters . . . . . . . . . . . . . . 46

Future Directions . . . . . . . . . . . . . . . . . . . . 49

\section{Introduction}

Globular star clusters (GCs) are among the oldest radiant objects in the universe. With typical masses $\sim 10^{4}-10^{6} \mathrm{M}_{\odot}$ (corresponding to luminosities of $\sim M_{V}=-5$ to -10 ) and compact sizes (half-light radii of a few pc), they are readily observable in external galaxies. The 15 years since the Annual Review by Harris (1991, "Globular Cluster Systems in Galaxies Beyond the Local Group") have seen a revolution in the field of extragalactic GCs. It is becoming increasingly apparent that GCs provide uniquely powerful diagnostics of fundamental parameters in a wide range of astrophysical processes. Observations of GCs are being used to constrain the star formation and assembly histories of galaxies, nucleosynthetic processes governing chemical evolution, the epoch and homogeneity of cosmic reionization, the role of dark matter in the formation of structure in the early universe, and the distribution of dark matter in present-day galaxies. GCs are valuable tools for theoretical and observational astronomy across a wide range of disciplines from cosmology to stellar spectroscopy.

It is not yet widely recognized outside the GC community that recent advances in GC research provide important constraints on galaxy formation that are complementary to in situ studies of galaxies at medium to high redshift. The theme of this article is the role of GC systems as tracers of galaxy formation and assembly, and one of our primary aims is to emphasize the current and potential links with results from galaxy surveys at high redshift and interpretations from stellar population synthesis, numerical simulations, and semi-analytical modeling. In what follows we will attempt to chronicle the observations that mark recent milestones of achievement and to place them in the wider theoretical and observational context. We will focus most closely on work carried out since about 2000. The preceding period is well-covered by the book of Ashman \& Zepf (1998) and the Saas-Fee lectures of Harris (2001). ${ }^{1}$ Among the significant topics not directly covered are young massive clusters (potential "proto-GCs"), X-ray sources in extragalactic GCs, and ultra-compact dwarf galaxies. Neither do we include a

\footnotetext{
${ }^{1}$ Available online at http://physwww.mcmaster.ca/ harris/Publications/saasfee.ps
} 
comprehensive discussion of the Galactic GC system.

The fundamental premise in what follows is that GCs are good tracers of the star formation histories of spheroids (early-type galaxies, spiral bulges, and halos), in the sense that major star-forming episodes are typically accompanied by significant GC formation. Low-level star formation (e.g., in quiescent galactic disks) tends to produce few, if any, GCs. Since most of the stellar mass in the local universe is in spheroids ( $~ 75 \%$; Fukugita, Hogan, \& Peebles 1998), GCs trace the bulk of the star formation history of the universe. Although the relationships between star formation, GC formation and GC survival are complex and do not necessarily maintain relative proportions under all conditions, this underlying assumption is supported by a number of lines of argument. Massive star clusters appear to form during all major star-forming events, such as those accompanying galaxy-galaxy interactions (e.g., Schweizer 2001). In these situations, the number of new clusters formed scales with the amount of gas involved in the interaction (e.g., Kissler-Patig, Forbes, \& Minniti 1998). The cluster formation efficiency (the fraction of star formation in clusters) scales with the star formation rate, at least in spiral galaxies where it can be directly measured at the present epoch (Larsen \& Richtler 2000). This may suggest that massive clusters form whenever the star-formation rate is high enough, and that this occurs principally during spheroid formation. Perhaps most importantly, the properties of GCs (especially their metallicities) are correlated with the properties of their host galaxies.

\section{Color Bimodality: Globular Cluster Subpopulations}

Perhaps the most significant development of the decade in the field of extragalactic GCs was the discovery that the color distributions of GC systems are typically bimodal. Indeed, color bimodality is the basic paradigm of modern GC studies. Nearly every massive galaxy studied to date with sufficiently accurate photometry has been shown to have a bimodal GC color distribution, indicating two subpopulations of GCs. In principle, these color differences can be due to age or metallicity differences or some combination of the two. Due to the well-known degeneracy between age and metallicity (e.g., Worthey 1994), the cause of this bimodality is not readily deduced from optical colors alone. Nonetheless, the significance of the finding was immediately recognized. The presence of bimodality indicates that there have been at least two major star-forming epochs (or mechanisms) in the histories of most - and possibly all - massive galaxies. Subsequent spectroscopic studies (see $\S 4$ ) have shown that color bimodality is due principally to a metallicity difference between two old subpopulations.

With our "bimodality-trained" modern eyes, we can see evidence of the phenomenon in the $B-I$ CFHT imaging of NGC 4472 in Couture, Harris \& Allwright (1991) and $C-T_{1}$ CTIO imaging of NGC 5128 by Harris et al. (1992). However, the first groups to propose bimodality (or "multimodality") were Zepf \& Ashman (1993) for NGC 4472 and NGC 5128 and Ostrov, Geisler, \& Forte (1993) for NGC 1399 (in fact, using the Harris et al. and Couture et al. colors). Observations of the GC systems of galaxies throughout the 1990s provided mounting evidence that bimodality was ubiquitous in massive galaxies. The primary catalyst of this research was the advent of the Hubble Space Telescope (HST). The Wide Field and Planetary Camera 2 (WFPC2) provided the spatial resolution and accurate photometry needed to reliably identify GC candidates in galaxies as distant as 
the Virgo Cluster at 17 Mpc (e.g., Whitmore et al. 1995). At this distance, GCs (with typical half-light radii of $2-3 \mathrm{pc} \sim 0.03-0.04^{\prime \prime}$ ) are resolvable with $H S T$ and their sizes are measurable with careful modeling of the PSF. This drove down the contamination from background galaxies and foreground stars to low levels and was a substantial improvement over multi-band optical photometry from the ground.

Among the larger and more comprehensive photometric studies using HST /WFPC2 were Gebhardt \& Kissler-Patig (1999), Larsen et al. (2001a) and Kundu \& Whitmore (2001a). Using data from the HST archive, Gebhardt \& Kissler-Patig showed that bimodality was a common phenomenon. However, since the imaging was shallow for many of the galaxies in their sample, they failed to find bimodality in $\sim 50 \%$ of their 50 galaxies. Taking advantage of deeper data, Larsen et al. (2001a) and Kundu \& Whitmore (2001a) found statistically significant bimodality in most of their sample galaxies, the majority of which were of earlytype. Galaxies that were tentatively identified as unimodal in these studies were later, with improved photometric precision, shown to conform to the bimodality "rule". Indeed, it is important to note that no massive elliptical (E) galaxy has been convincingly shown to lack GC subpopulations. An absence of metal-poor GCs was suggested for both NGC 3311 (Secker et al. 1995) and IC 4051 (Woodworth \& Harris 2000), and an absence of metal-rich GCs for NGC 4874 (Harris et al. 2000). However, HST/WFPC2 imaging of NGC 3311 (Brodie, Larsen, \& Kissler-Patig 2000) revealed a healthy subpopulation of metal-poor GCs. It is now clear that the WFPC2 photometry of the Coma E IC 4051 was not deep enough to securely argue for a uni- or bimodal fit. Finally, the NGC 4874 result was due to a photometric zeropoint error (W. Harris, private communication). The discovery of a massive E which indeed lacked a metal-poor (or metal-rich) subpopulation would be important, but so far no such instances have been confirmed.

The majority of these HST studies were carried out in $V$ - and $I$-equivalent bands. This choice was largely driven by efficiency considerations (shorter exposure times needed to reach a nominal $\mathrm{S} / \mathrm{N}$ ), despite the fact that other colors, such as $B-I$, offer much better metallicity sensitivity for old stellar populations.

It has been known for some time that the GC system of the Milky Way is also bimodal. The presence of GC subpopulations in the Milky Way was codified by Zinn (1985; see also Armandroff \& Zinn 1988) who identified two groups of GCs. "Halo" GCs are metal-poor, non-rotating (as a system), and can be found at large galactocentric radii. "Disk" GCs are metal-rich and form a flattened, rotating population. Later work on the spatial and kinematic properties of the metal-rich GCs by Minniti (1995) and Côté (1999) identified them with the Milky Way bulge rather than its disk (as we shall see below, this association seems to hold for other spirals as well, although see the discussion in $§ 3.3$ ). In addition to their sample of early-type galaxies, Larsen et al. (2001a) also discussed the GC systems of the Milky Way and NGC 4594 in some detail, pointing out that the locations of the GC color peaks in these spirals were indistinguishable from those of massive early-type galaxies.

The blue (metal-poor) and red (metal-rich) peaks in massive early type galaxies typically occur at $V-I=0.95 \pm 0.02$ and $1.18 \pm 0.04$ (Larsen et al. 2001a). These colors correspond to $[\mathrm{Fe} / \mathrm{H}] \sim-1.5$ and -0.5 for old $\mathrm{GCs}$ (or a bit more metalrich, depending on the metallicity scale and color-metallicity relation adopted). Figure 1 shows a histogram of the $V-I$ colors of GCs in the Virgo gE M87, which 
clearly shows bimodality (Larsen et al. 2001a). However, the peak locations are not exactly the same for all galaxies. Before GC bimodality was discovered, van den Bergh (1975) suggested and Brodie \& Huchra (1991) confirmed a correlation between the mean color/metallicity of GC systems and the luminosity of their parent galaxies. Brodie \& Huchra (1991) also showed that the slope of this relation was very similar to the relation connecting galaxy color and galaxy luminosity, but the GC relation was offset toward lower metallicities by about 0.5 dex. They noted that the similarity in slope suggests a close connection between the physical processes responsible for the formation of both GCs and galaxies. Subsequently, a correlation between the color of just the metal-rich GCs and host galaxy luminosity was found by Forbes et al. (1997), Larsen et al. (2001a), and Forbes \& Forte (2001). The slope of this relation was again found to be similar to that of the color-magnitude relation for early-type galaxies $\left(V-I \propto-0.018 M_{V}\right)$, suggesting that metal-rich GCs formed along with the bulk of the field stars in their parent galaxies.

With the exception of Larsen et al. (2001a), little or no correlation between the color of the metal-poor GCs and host galaxy luminosity was reported in these studies, although Burgarella et al. (2001) and Lotz, Miller, \& Ferguson (2004) suggested such a relation might be present, but only for the dwarf galaxies. Larsen et al. found a shallow relation for the metal-poor GCs in their sample of 17 massive early-type galaxies, albeit at moderate $(3 \sigma)$ statistical significance. Strader, Brodie, \& Forbes (2004a) compiled and reanalyzed high-quality data from the literature and found a significant $(>5 \sigma)$ correlation for metal-poor GCs, extending from massive Es to dwarfs over $\sim 10$ magnitudes in galaxy luminosity. The relation is indeed relatively shallow $\left(V-I \propto-0.009 M_{V}\right.$, or $\left.Z \sim L^{0.15}\right)$, making it difficult to detect, especially in heterogeneous data sets. This same slope was confirmed by Strader et al. (2006) and Peng et al. (2006) for earlytype galaxies in Virgo. Figure 2 shows $[\mathrm{Fe} / \mathrm{H}]$ vs. $M_{B}$ for both subpopulations; the GC peaks are taken from Strader et al. (2004a) and Strader et al. (2006) and have been converted from $V-I$ and $g-z$ using the relations of Barmby et al. (2000) and Peng et al. (2006), respectively. These data, together with ancillary information about the GC systems, are compiled in Table 1. The true scatter at fixed $M_{B}$ is unclear, since the observational errors vary among galaxies, and there may an additional component due to small differences between the $V-I$ and $g-z$ color-metallicity relations. The cutoff in the metal-rich relation at $M_{B} \sim-15.5$ primarily reflects the magnitude limit of the sample; it may continue to fainter magnitudes, although many such galaxies have only metal-poor GCs. The remarkable inference to be drawn from Figure 2 is that the peak metallicities of both subpopulations are determined primarily by galaxy luminosity (or mass) across the entire spectrum of galaxy types.

Using their new color-metallicity transformation between $g-z$ and $[\mathrm{Fe} / \mathrm{H}]$, Peng et al. found $Z \sim L^{0.25}$ for metal-rich GCs, which is also consistent with the previous estimates of the slope already noted. The color-metallicity relation appears to be quite nonlinear, as discussed below. Thus, even though the slopes of the metal-poor and metal-rich relations are significantly different in the GC colorgalaxy luminosity plane, they are similar in the GC metallicity-galaxy luminosity plane (see Figure 2). In $\S 11$ we discuss the constraints on galaxy formation implicit in these relations.

The Advanced Camera for Surveys (ACS) on HST has significantly advanced our understanding of the color distributions of GC systems, offering a wider field 
of view and improved photometric accuracy compared to WFPC2. Three large studies of Es utilizing HST/ACS have recently been published. As mentioned above, Peng et al. (2006) and Strader et al. (2006) studied the GC systems of early-type galaxies (ranging from dwarf to giant) using $g$ and $z$ data taken as part of the ACS Virgo Cluster Survey (Côté et al. 2004). Peng et al. investigated all 100 (E and S0) galaxies, while Strader et al. focused solely on the ellipticals (Es). Harris et al. (2006) used BI ACS photometry to analyze GCs in eight "BCGs", galaxies which are among the brightest in their respective groups or clusters.

These studies resulted in several new discoveries. First, a correlation was found between color and luminosity for individual metal-poor GCs in some giant Es (the "blue tilt"; see Figure 3). This is the first detection of a mass-metallicity relation for GCs. The blue tilt was found by Strader et al. in the Virgo giant Es (gEs) M87 and NGC 4649 and by Harris et al. in their sample, although the interpretations of the findings differ. The mass-metallicity relation for individual metal-poor GCs may argue for self-enrichment. Strader et al. speculated that these metalpoor GCs were able to self-enrich because they once possessed dark matter halos that were subsequently stripped (see also discussion in $\$ 12$ ). The M87 data are well-fit by a relation equivalent to $Z \propto M^{0.48}$ over the magnitude range $20<z<23.2$, where the turnover of the GC luminosity function (GCLF) is at $z \sim 23$. Harris et al. found a similar relation $\left(Z \propto M^{0.55}\right)$ but suggested that the trend was only present at bright luminosities $\left(M_{I} \lesssim-9.5\right.$ to -10 , corresponding to $z \lesssim 22$ in the Strader et al. Virgo dataset). The CMDs in Strader et al. for M87 and NGC 4649 appear consistent with a continuation of the correlation to magnitudes fainter than $z=22$, but do not strongly distinguish between the two interpretations. We do know that the blue tilt phenomenon is not confined to galaxies in high density environments or even just to $\mathrm{E}$ galaxies. It has recently been reported for NGC 4594 (Spitler et al. 2006), a luminous Sa galaxy that lies in a loose group. Curiously, the Virgo gE NGC 4472 (also studied by Strader et al.) shows no evidence for the blue tilt. If this lack of a tilt is confirmed with better data, it will be a strong constraint on any potential "universal" model for explaining the phenomenon in massive galaxies. The Milky Way itself does not show evidence for the tilt, but this could be due to the small number of metal-poor GCs $(\sim 100)$ compared to massive galaxies or to the inhomogeneity of metallicities and integrated photometry in current catalogs.

Harris et al. (2006) found that the metal-poor GC sequence lay slightly redward of, but parallel to, the luminosity-color sequence of $\mathrm{dE}$ nuclei from Lotz et al. (2004). The dE data were converted from $V-I$ to $B-I$ for this comparison, and a small zero-point shift in the color conversion would line these up. Indeed, in Figure 3 the M87 $z$ vs. $g-z$ color-magnitude diagram is shown superimposed with dE nuclei from Strader et al. (2006). Here the sequence of dE nuclei falls right on top of the bright end of the metal-poor GC sequence, although the $\mathrm{dE}$ sequence is broader. This could be consistent with a scenario in which at least some of the metal-poor GCs in gEs are stripped nuclei of dEs. Since the size range of $\mathrm{dE}$ nuclei overlaps that of GCs, it may be impossible to ascertain the provenance of every luminous cluster.

Harris et al. suggested that the tilted metal-poor GC relation caused the metalpoor and metal-rich peaks to merge at the brightest GC luminosities, turning a bimodal distribution into a nominally unimodal one. By contrast, Strader et al. argued that at these high luminosities there is a separate population of objects with larger-than-average sizes and a range of colors, spanning the metal- 
poor to metal-rich subpopulations. Indeed, Harris et al. find that $\sim 20-30$ of the brightest objects in the nearest galaxy in their sample, NGC 1407 (at $\sim 21$ $\mathrm{Mpc}$ ), appear to be extended with respect to normal GCs. The size measurements suggest that there is something qualitatively different about (at least) a subset of the brightest GCs, which has also been recognized in NGC 1399 (Dirsch et al. 2003) and NGC 4636 (Dirsch, Schuberth, \& Richtler 2005). The sizes and luminosities of the bright intermediate-color objects in these galaxies suggest a relation to the "ultra-compact dwarf" galaxies (UCDs) discovered in both the Fornax and Virgo clusters (e.g., Phillips et al. 2001).

The second significant finding was that the color dispersion of the metal-rich GCs is nearly twice as large as that of the metal-poor GCs. Peng et al., Strader et al., and Harris et al. reported essentially the same dispersions in the color distributions of both subpopulations. However, Peng et al. and Harris et al. adopted different color-metallicity relations, and these led to divergent conclusions about the metallicity distributions of these populations. Harris et al. fit a linear relation between $B-I$ and metallicity using Galactic GCs. This has the advantage of being independent of stellar population models but the disadvantage of being yoked to the metallicity distribution of Galactic clusters. There are no low-reddening Galactic GCs with $[\mathrm{Fe} / \mathrm{H}] \gtrsim-0.5$, so the empirical relation is unconstrained at these metallicities, and the data are poorly fit by a linear relation in the very metal-poor regime. Peng et al. used a piecewise linear relation broken at $g-z=1.05$ or $[\mathrm{Fe} / \mathrm{H}] \sim-0.8$. This utilized GCs in the Galaxy, M87, and NGC 4472 with both $g-z$ colors and spectroscopic metallicities (these are still ultimately tied to the Galactic $\mathrm{GC}[\mathrm{Fe} / \mathrm{H}]$ scale). The qualitative effect was to "flatten" the relation at low metallicities compared to a linear fit - so small color changes correspond to large metallicity changes - and to "steepen" it at high metallicities.

Consequently, Peng et al. found the metal-poor GCs to have a larger metallicity dispersion than the metal-rich GCs: $68 \%$ half-width $[\mathrm{Fe} / \mathrm{H}]$ intervals of $\sim 0.6$ dex and 0.3 dex for the metal-poor and metal-rich GCs in massive galaxies, respectively (these were derived from a nonparametric analysis and thus are not exactly equivalent to a $1 \sigma$ dispersion for a normal distribution). By contrast, Harris et al. deduced mean $1 \sigma$ dispersions of $\sim 0.3$ dex and 0.4 dex, and noted that the metal-poor and metal-rich subpopulations in the Galaxy have $\sigma=0.34$ dex and $\sigma=0.16$ dex. The relative widths of the two Galaxy subpopulations are more consistent with the Peng et al. results, though smaller in an absolute sense. However, even if the metallicity dispersion in the metal-poor GCs is larger, the absolute metallicity spread is much smaller. The implications of these differences for the enrichment histories of the two subpopulations remain to be seen.

It seems clear from the new Galactic GC data in Peng et al. that a single linear fit is not optimal, but the exact form of the relation at metallicity extremes is poorly constrained. Clearly, identifying the correct form of the $g-z$ to $[\mathrm{Fe} / \mathrm{H}]$ conversion (and, indeed, conversions for other colors) is essential, since the metallicity spreads in individual subpopulations have important implications for the formation and assembly histories of GC systems. The different color dispersions for the subpopulations also indicate that it is necessary to use heteroscedastic (unequal variance) fits in mixture modeling. Homoscedastic fits will give systematic errors in the peak values, but may be the best option for systems with few GCs. Another important effect of nonlinear color-metallicity relations is that bimodal color distributions can be enhanced or even created from metallicity distributions 
that are not strongly bimodal (Richtler 2006; Yoon, Yi, \& Lee 2006).

The last principal finding in these new studies, reported in both Strader et al. and Peng et al., is that many dEs have metal-rich GC subpopulations. Their colors are consistent with an extrapolation of the GC color-galaxy luminosity relation for massive galaxies, though the fraction of metal-rich GCs tends to be smaller in dEs than in massive Es. Peng et al. found that the median fraction of metal-rich GCs is $\sim 0.15-0.2$ in dEs and rises steeply towards more luminous galaxies. Some of this increase is due to the different radial distributions of the two subpopulations; the $H S T$ /ACS data preferentially sample the more centrallyconcentrated metal-rich GCs. Global fractions of metal-rich GCs in massive Es are likely to be closer to $0.3-0.4$ (Rhode \& Zepf 2004). The changing fraction of metal-rich GCs with galaxy luminosity, combined with the correlations of GC colors with galaxy luminosity for both subpopulations, fully explains the classic correlation between the mean color/metallicity of a GC system and parent galaxy luminosity (Brodie \& Huchra 1991). The overall slope actually measured depends on how the sample is defined. If HST data are used, the metal-rich GCs will be overrepresented and the steep slope of the metal-rich GC relation will dominate the overall relation.

These new results suggest that considerable undiscovered detail may still be hidden in GC color distributions. Intensive use should be made of $H S T$ /ACS while it is still operational. It is likely to be the best instrument available for the next $\sim 15$ years for studying the optical color distributions of GCs.

\subsection{Scenarios for Bimodality}

Once bimodality was observed to be a common phenomenon, several scenarios were presented to explain it. In this section, we briefly describe the leading scenarios (see also the review of West et al. 2004), but leave detailed discussion until §11. The major merger model of Ashman \& Zepf (1992) has the distinction of being the only model to predict bimodality before it was observed. This model evolved from early work suggesting that $\mathrm{E}$ galaxies formed in gas-rich major mergers of disk galaxies (Toomre \& Toomre 1972; Toomre 1977; Schweizer 1987). Burstein (1987) and Schweizer (1987) suggested that new GCs might be formed in large quantities during the merger process. Ashman \& Zepf (1992) and Zepf \& Ashman (1993) developed this idea into a predictive model in which the metalpoor GCs are donated by the progenitor spirals and the metal-rich GCs are formed in the gas-rich merger. This model gained enormous support when new HST observations of merging galaxies found large numbers of young massive star clusters (YMCs). The most famous example of this is the Antennae (Whitmore \& Schweizer 1995), but several other cases were discovered in the early-to-mid 1990s (e.g., NGC 1275, Holtzman et al. 1992; NGC 7252, Miller et al. 1997). The interpretation of these YMCs as "proto-GCs" was widely adopted. Determining the extent to which these YMCs have properties consistent with "normal" old GCs is still an active area of research; see $\S 8$ below.

Several problems with the major merger model were pointed out in Forbes, Brodie \& Grillmair (1997), who showed that, when examined in detail, the number and color distributions of GCs in massive Es appeared to be inconsistent with the merger model predictions (see $\S 11.1 .1$ ). They instead suggested that bimodality could arise as a consequence of a multi-phase dissipational collapse. In their scenario, the metal-poor globular clusters were formed in gaseous frag- 
ments during the earliest phases of galaxy formation. GC formation was then truncated at high redshift and resumed after a dormant period of a few Gyr. During this second phase the metal-rich GCs and the bulk of the galaxy field stars were formed. Forbes et al. discussed this truncation process in terms of feedback, with gas being expelled from the early cluster-forming clumps. Such gas would later cool and recollapse into the more fully-formed galactic gravitational potential until the local conditions were conducive to renewed star formation. Subsequently, Santos (2003) suggested cosmic reionization as the mechanism for truncating metal-poor GC formation. Although some details differ, similar "in situ" models for GC formation were also presented by Harris \& Pudritz (1994) and Harris, Harris, \& Poole (1999).

In the accretion scenario of Côté, Marzke \& West (1998), the metal-rich GCs were formed in situ in a massive seed galaxy, while the metal-poor GCs were acquired in the dissipationless accretion of neighboring lower-mass galaxies (see also Hilker 1998; Hilker, Infante, \& Richtler 1999, and earlier work by Muzzio 1987 and references therein). This works in principle because of the long-known relation between the mean metallicity of the GC system and the mass of the host galaxy (van den Bergh 1975; Brodie \& Huchra 1991; Forbes \& Forte 2001). Stripping of GCs without the accompanying galaxy light is also a possibility in dense clusters. Côté et al. (1998; 2000; 2002) explored the accretion model in detail using Monte Carlo simulations. They showed that the bimodality observed in massive galaxies can be reproduced provided that (i) each galaxy has an intrinsic "zero-age" population of GCs, whose metallicity increases with the galaxy's mass, and (ii) the primordial galactic mass function for low-mass galaxies is a rather steep power law (with $\alpha \sim-2$ ). The slope is consistent with the halo mass functions predicted by standard $\Lambda$ CDM models, but much steeper than that actually observed for present-day low-mass galaxies (but see $\S 11.2$ ).

This triad of scenarios: major merger, in situ/multiphase, and accretion, were the ones most frequently discussed to explain bimodality throughout the last decade. In a very real sense, the distinctions between these models could be blurred by placing the merger or accretion events at high redshift and allowing for significant gas in the components, so to some extent the debate was semantic. Indeed, Harris (2003) noted that these scenarios could be reclassified in terms of the amount of gas involved. Nevertheless, we have presented these scenarios here to give some context for the discussion of observations that follows.

\section{Global Properties}

\subsection{Specific Frequency}

Harris \& van den Bergh (1981) introduced specific frequency $\left(S_{N}=N_{G C} \times\right.$ $\left.10^{0.4\left(M_{V}+15\right)}\right)$ as a measure of the richness of a GC system normalized to host galaxy luminosity. This statistic has been widely used in toy models to assess the feasibility of galaxy formation mechanisms, e.g., the formation of gEs from disk-disk mergers. $N_{G C}$ was originally calculated by doubling the number of GCs brighter than the turnover of the GCLF. This definition makes both practical and physical sense. The faint end of the GCLF is usually poorly defined (suffering both contamination and incompleteness), and $\sim 90 \%$ of the mass of the GC system resides in the bright half. This approach is implicit when fitting a Gaussian (or $t_{5}$ ) functions to observed GCLFs, since observations rarely sample the faintest 
clusters.

$S_{N}$ comparisons among galaxies are only valid if all galaxies have the same stellar mass-to-light (M/L) ratios. For this reason, Zepf \& Ashman (1993) introduced the quantity $T$ - the number of GCs per $10^{9} M_{\odot}$ of galaxy stellar mass. Since M/L is not generally known in detail for a particular galaxy, it is usually applied as a scaling factor that is different for each galaxy type, e.g., stellar $M / L_{V}=10$ for Es and 5 for Sbc galaxies like the Galaxy. In this section we quote observational results in terms of $S_{N}$, but convert to $T$ for comparisons among galaxies. An even better approach would be to directly estimate stellar masses for individual galaxies. Olsen et al. (2004) discuss the use of $K$-band magnitudes for this purpose.

Soon after the merger model was proposed, van den Bergh (1982) argued that elliptical galaxies could not arise from the merger of spiral galaxies, since spirals have systematically lower $S_{N}$ values than ellipticals. Schweizer (1987) and Ashman \& Zepf (1992) suggested that this problem could be solved if new GCs were formed in the merging process. Observations of large numbers of YMCs in recent mergers seemed to support this solution. However, the $S_{N}$ will only increase through the merger if the GC formation efficiency is higher than it was when the existing GC system was formed. Studies of YMCs in nearby spirals (Larsen \& Richtler 2000) suggest that galaxies with larger star formation rates have more of their light in young clusters, but it seems unlikely that local mergers are more efficient at forming GCs than in the gas-rich, violent early universe (see Harris 2001).

In recent years there have been few wide-field imaging studies of the sort needed to accurately estimate $S_{N}$. Nonetheless, a trend has emerged that suggests reconsideration of some earlier results. Newer $S_{N}$ values tend to be lower than older ones. This revision stems, for the most part, from improved photometry that is now deep enough to properly define the GCLF turnover and reject contaminants. Imaging studies that cover a wide field of view are also important, because they avoid uncertain extrapolations of spatial profiles from the inner regions of galaxies. In some cases, like the Fornax gE NGC 1399, the luminosity of the galaxy was underestimated. When corrected, the $S_{N}$ value for this galaxy was revised downward by a factor of two, from $S_{N} \sim 12$ to $5-6$ (Ostrov et al. 1998; Dirsch et al. 2003).

It has been argued for some time that the evolutionary histories of central cluster galaxies are different than other Es of similar mass. Somehow this special status has resulted in high $S_{N}$ (or $T$ ). In addition, galaxies in high-density environments tend to have higher $S_{N}$ than those in groups. McLaughlin (1999) argued that high $S_{N}$ values in central galaxies arise because bound hot gas has been ignored for these galaxies, and that they do not reflect an increased GC formation efficiency with respect to other galaxies (see Blakeslee, Tonry, \& Metzger 1997 for additional arguments in favor of the hypothesis that high $S_{N}$ is due to large galactic mass-to-light ratios). The properties of the E galaxy NGC 4636 may also be consistent with this view. Despite its relatively low-density environment, it has $S_{N} \sim 6$, a value typical of central cluster galaxies (Dirsch et al. 2005). However, the galaxy has a dark matter halo (traced by a halo of hot X-ray gas) that is unusually massive for its luminosity.

The classic Harris (1991) diagram of $S_{N}$ vs. luminosity implied a monotonic increase of $S_{N}$ with galaxy mass for high-mass galaxies; that diagram also showed an increase toward low luminosities for dwarf galaxies. Both of these trends are 
less apparent in newer data. The situation for dwarf galaxies is discussed in more detail in $\S 10$, though it is worth noting here that it is difficult to make robust estimates of the number of GCs in low-mass galaxies outside of the Local Group. In our view, the run of $S_{N}$ with galaxy mass and environment remains uncertain. Additional discussion may be found in McLaughlin (1999) and the reviews by Elmegreen (1999) and Harris (2001).

3.1.1 Subpopulations Many of the problems with direct $S_{N}$ comparisons can be circumvented by considering the metal-poor and metal-rich subpopulations separately. In particular, recent mergers should only affect the metal-rich peak; independent of the details of new star formation, the $S_{N}$ of metal-poor GCs will not increase. Rhode, Zepf, \& Santos (2005) have exploited this fact by studying $T$ for metal-poor GCs in 13 massive nearby galaxies, nearly equally split between early- and late-type. In Figure 4 we show both $T_{b l u e}$ and $T_{r e d}$ vs. stellar galaxy mass. The $T_{b l u e}$ values are taken from their paper, and the $T_{r e d}$ data were kindly provided by K. Rhode. Rhode et al. found an overall correlation between $T_{\text {blue }}$ and galaxy mass. The spirals are all consistent with $T_{\text {blue }} \sim 1$, while cluster Es lie higher at $T_{\text {blue }} \sim 2-2.5$. NGC 4594 also has $T_{\text {blue }} \sim 2$ (note in their classification NGC 4594 is an S0, not an Sa). Other field/group Es, including NGC 5128, NGC 1052, and NGC 3379, have values similar to those of the spirals. Since $M / L_{V}$ increases with galaxy mass (with a relation as steep as $\propto L^{0.10}$; e.g., Zepf \& Silk 1996), it is reasonable to expect at least a weak trend of $T$ with galaxy mass. However, Rhode et al. (2005) argue that this can account for only $\sim 1 / 3$ of the observed trend. Because no global GC color studies of M87 have been published, this galaxy was not included in the Rhode et al. study. Its $S_{N}$ value is $\sim 3$ times larger than the Virgo gE NGC 4472 (Harris, Harris \& McLaughlin 1998). If both galaxies have similar total fractions of metal-poor GCs, the $T_{b l u e}$ value for M87 would be $\sim 8$, though this value would be much lower if the mass of its hot gas halo were included along with the stellar mass (McLaughlin 1999).

Based solely on the metal-poor GCs, these comparisons seem to rule out the formation of cluster gEs (and some massive Es in lower-density environments) by major mergers of disk galaxies. However, the relative roles of galaxy mass and environment are still unclear. Spirals in clusters like Virgo and Fornax and more massive field/small group Es remain to be studied in detail. The metal-poor GC subpopulations of some lower-mass Es in low-density environments are still consistent with merger formation. The biggest caveat to these interpretations is the effect of biasing - that structure formation is not self-similar. Presentday spirals are mostly located in low-density environments like loose groups and the outskirts of clusters. High- $T_{\text {blue }}$ disk galaxies may have been common in the central regions of proto-galaxy clusters at high redshift but have merged themselves out of existence (or could, in some cases, have been converted to S0s) by the present day. Rhode et al. (2005) argue that the observed trend of high $T_{\text {blue }}$ for cluster galaxies might be expected in hierarchical structure formation, since halos in high-density environments will collapse and form metal-poor GCs first (see also West 1993). If GC formation is then truncated (by reionization, for example), such halos will have a larger number of metal-poor GCs than similar mass halos in lower density environments. See $\S 11$ for additional discussion of biasing.

The $T_{\text {red }}$ data show a similar correlation with galaxy mass, although with a smaller dynamic range. Note that the $T_{\text {red }}$ values for the spirals $(\sim 0.5-1)$ are 
normalized to the total galaxy mass in stars. Most have bulge-to-total ratios of $<0.3$, so if $T_{\text {red }}$ had been normalized just to the spheroidal component, it would be substantially higher than plotted. These data appear consistent with the hypothesis of near-constant formation efficiency for metal-rich GCs in both spirals and field Es with respect to spheroidal stellar mass (Forbes, Brodie, \& Larsen 2001; see also Kissler-Patig et al. 1997). The massive cluster Es have both higher $T_{\text {red }}$ and $T_{\text {blue }}$. Again, however, because of the sample under study (with few field Es and no cluster spirals), it is unclear whether environment or mass is the predominating influence. This distinction is moot for the most massive Es since they are found almost exclusively in clusters, but is still relevant for typical Es.

Estimating the spread in $T_{r e d}$ at a given galaxy mass should help constrain the star-formation histories of early-type galaxies. The stellar mass of an E might have been built entirely through violent, gas-rich mergers (with metal-rich GC formation), or, alternatively, many of the stars could instead have formed quiescently in mature spiral disks (with little metal-rich GC formation). $T_{\text {red }}$ for the latter E should be significantly lower than for the former E.

\subsection{Radial and Azimuthal Distributions}

Most of the existing information on the global spatial distributions of GCs dates from older studies that could not separate GCs into subpopulations. The projected radial distributions are often fitted with power laws over a restricted range in radius, and it is clear that more luminous galaxies have shallower radial distributions (Harris 1986; see the compilation in Ashman \& Zepf 1998). Considering the GC system as a whole, typical projected power law indices range from $\sim-2$ to -2.5 for some low-luminosity Es (this is also a good fit to the Galactic GC system; Harris 2001) to $\sim-1.5$ or a bit lower for the most massive gEs. However, it should be kept in mind that power laws provide a poor fit over the entire radial range. Most GC radial distributions have cores, and gradually become steeper in their outer parts. King models capture some of this behavior. In nearly all cases, the GCs have a more extended spatial distribution than the galaxy field stars.

There have been a few wide field imaging programs which considered GC subpopulations separately. In their study of the Virgo gE NGC 4472, Geisler et al. (1996) were the first to show clearly that color gradients in GC systems are driven solely by the different radial distributions of the subpopulations. The metal-poor and metal-rich GCs themselves show no radial color gradients. Rhode \& Zepf (2001) found a color gradient for the total GC system in NGC 4472 interior to $\sim 8^{\prime}$, but no gradient when the full radial extent of the GC system $\left(22^{\prime} \sim 110 \mathrm{kpc}\right)$ was considered. Dirsch et al. (2003) studied the GC system of the Fornax gE NGC 1399 out to $\sim 25^{\prime}(\sim 135 \mathrm{kpc})$; this work was extended to larger radii by Bassino et al. (2006). The radial distributions of the two subpopulations are shown in Figure 5, along with the profile of the galaxy itself. The metal-poor and metal-rich subpopulations have power law slopes of $\sim-1.6$ and $\sim-1.9$, respectively. These differences persist to large radii, and lead to an overall color gradient in the GC system. The radial distribution of the metal-rich GCs is a close match to that of the galaxy light, and suggests that they formed contemporaneously.

Another notable finding is the rather abrupt truncation of GC systems at large galactocentric radius. Rhode \& Zepf (2001) found that the surface density of GCs 
in NGC 4472 falls off faster than a de Vaucoleurs or power law fit at $\sim 20^{\prime}(\sim 100$ kpc). A similar drop-off occurs at 11' in NGC 4406 (Rhode \& Zepf 2004), and at $9^{\prime}$ in NGC 4636 (Dirsch et al. 2005). This may be evidence of truncation by the tidal field of the cluster.

Dissipationless mergers (whether disk-disk or E-E) tend to flatten the radial slopes of existing GC systems and create central cores (Bekki \& Forbes 2005). These cores are observed and have sizes of a few kpc (Forbes et al. 1996). These results apply to pre-existing GCs, which certainly includes metal-poor GCs, as well as metal-rich GCs that existed before the merger. Thus, the trends predicted by the Bekki \& Forbes simulation appear qualitatively consistent with observations. The observations are also consistent with more extensive merger histories for more massive galaxies; these gradually produce larger cores and flatter GC radial distributions. A desirable extension to this work would be to place these simulations in a cosmological framework in which merging occurs according to a full N-body merger tree. Also, as data become available, comparisons between observations and simulations could be restricted to metal-poor GCs. With this approach, any new metal-rich GCs that might have formed in the merger are irrelevant.

Ashman \& Zepf (1998) noted that little was known about the two-dimensional spatial distributions of GC systems. Scant progress has been made in the intervening years. Existing data are consistent with the hypothesis that both subpopulations have ellipticities and position angles similar to those of the spheroids of their parent galaxies (e.g., NGC 1427, Forte et al. 2001; NGC 1399, Dirsch et al. 2003; NGC 4374, Gómez \& Richtler 2004; NGC 4636, Dirsch et al. 2005). This also holds for the dEs in the Local Group (Minniti et al. 1996). It may be that in some galaxies (e.g., the E4 NGC 1052; Forbes, Georgakakis, \& Brodie 2001) the metal-rich GCs follow the galaxy ellipticity more closely than the metal-poor GCs, but whether this is a common phenomenon is unknown. Naively, if the metal-rich GCs formed along with the bulk of the galaxy field stars, they should closely trace the galaxy light. The spatial distribution of the metal-poor GCs will depend in detail upon the assembly history of the galaxy.

\subsection{Variations with Galaxy Morphology}

3.3.1 SpIRAls Our views on the GC systems of spiral galaxies are heavily shaped by the properties of the Milky Way (and, to a lesser degree, M31). This is discussed in detail in $§ 5$. A principal result from the Galaxy is that the metal-poor and metal-rich GCs are primarily associated with the halo and bulge, respectively. Forbes et al. (2001) introduced the idea that the bulge GCs in spirals are analogous to the "normal" metal-rich GCs in early-type galaxies, and that in both spirals and field Es the metal-rich GCs have $S_{N} \sim 1$ when normalized solely to the bulge luminosity. The constancy of bulge $S_{N}$ appeared consistent with observations of a rather small set of galaxies (Milky Way, M31, M33, NGC 4594) but needed further testing.

Goudfrooij et al. (2003) provided such a test with an HST/WFPC2 imaging study of seven edge-on spirals, ranging across the Hubble sequence (the previously-studied NGC 4594 was part of the sample). Edge-on galaxies were chosen to minimize the effects of dust and background inhomogeneities on GC detection. Corrections for spatial coverage were carried out by comparison to the Galactic GC system; this does not appear to introduce systematic errors (see 
Goudfrooij et al. for additional discussion). Kissler-Patig et al. (1999) had previously found that one of these galaxies, NGC 4565, has a total number of GCs similar to the Galaxy. The small WFPC2 field of view and the low $S_{N}$ (or $T$ ) values of spirals as compared to Es resulted in the detection of only tens of GCs in some of the Goudfrooij et al. galaxies. While bimodality was not obvious in all of the color distributions, each galaxy had GCs with a range of colors, consistent with multiple subpopulations. Using a color cut to divide the samples into metal-poor and metal-rich GCs, Goudfrooij et al. found that all of the galaxies in their study had (i) a subpopulation of metal-poor GCs with $S_{N} \sim 0.5-0.6$ (normalized to total galaxy light), and (ii) constant bulge $S_{N}$, with the exception of the rather low-luminosity Sa galaxy NGC 7814, which appeared to have few GCs of any color. NGC 7814 was later the target of a ground-based study by Rhode \& Zepf (2003) with the WIYN telescope. They found a significant metal-rich GC subpopulation with a bulge $S_{N}$ squarely in the middle of the range found for the other galaxies, which showed that the single WFPC2 pointing on the sparse GC system of NGC 7814 gave an incomplete picture of the galaxy. In this case even small radial leverage was important: Rhode \& Zepf found that the surface density of GCs dropped to zero at just $12 \mathrm{kpc}\left(3^{\prime}\right)$ projected.

Chandar, Whitmore, \& Lee (2004) studied GCs in five nearby spirals. Their galaxies (e.g., M51, M81) tended to be closer and generally better-studied than those in the Goudfrooij et al. sample, but they were also at less favorable inclination angles. As a result, their GC candidate samples were more prone to contamination and more affected by (sometimes unknown amounts of) reddening. This was partially mitigated by their wide wavelength coverage, including (in some cases) $U$-band imaging, to help constrain the reddening of individual GCs. Chandar et al. found evidence for bimodal GC color distributions in M81 and M101. Interestingly, M51, which had rather deep imaging, showed no evidence for metal-rich GCs. They found that NGC 6946 and M101 had subpopulations of clusters with sizes similar to GCs but extending to fainter magnitudes; the typical log-normal GCLF was not seen. In NGC 6946 the imaging was quite shallow and these faint objects were found to be blue, suggesting that they might be contaminants. However, in M101 the imaging was deeper and the faint objects had red colors, consistent with old stellar populations. This may be evidence that some spirals possess clusters unlike those typical of Es, and these clusters may be related to the faint red objects seen in some S0s (see next subsection). Chandar et al. also compiled data from the literature on the GC systems of spirals, and argued that $S_{N} / T$ depends on Hubble type, but not on galaxy mass. This is consistent with the findings of Goudfrooij et al. (2003).

In principle, the $S_{N}$ value should depend on whether a particular bulge formed "classically", with intense star formation, or through secular processes in quiescent gas disks (e.g., Kormendy \& Kennicutt 2004). In the former case we might expect a bulge $S_{N}$ similar to that found for Es, but in the latter case the star formation is likely to be sufficiently slow and extended that few or no GCs are formed along with the bulge stars. This may result in a rather low bulge $S_{N}$ compared to Es of similar mass. The implication is that all the bulges of galaxies in the Goudfrooij et al. sample formed predominantly through the "classical" route. The generally old ages of the metal-rich GCs in spirals (see §4) is evidence that the majority of bulge star formation, by whatever mechanism, happened at relatively early epochs.

Kormendy \& Kennicutt (2004) argue that a large fraction of spiral bulges are 
built by secular evolution, and that these "pseudobulges" are especially common in late-type spirals. The diagnostics for pseudobulges are many, but include cold kinematics, surface brightness profiles with low Sersic indices, and, in some cases, young stellar populations. It is worth emphasizing that many of these pseudobulges could be composite bulges with young to intermediate-age stars superposed on an old classical bulge. In this case, the bulge $S_{N}$ could serve as a diagnostic of the degree to which a given bulge can have been built by classical or secular processes. The Milky Way itself could be an example of such a bulge. Its bulge is dominated by an old stellar population, but has a rather low velocity dispersion for its mass. The kinematics of the metal-rich GCs could be consistent with association with either a bulge or a bar (Côté 1999).

3.3.2 S0s The leading theory for the formation of most S0s involves their transformation from spirals as groups and clusters virialize (e.g., Dressler et al. 1997). This can occur in a variety of ways, including ram pressure stripping and minor mergers that disrupt the disk sufficiently to halt star formation. In this context, it may be more appropriate to compare the GC systems of S0s to those of spirals, rather than make the traditional comparison with Es. Nonetheless, the GC systems of S0s appear to be quite similar to those of Es when compared at fixed mass. Kundu \& Whitmore (2001b) studied a variety of S0s with WFPC2 snapshot imaging, and in many galaxies found broad color distributions consistent with multiple subpopulations. Peng et al. (2006b) used deeper imaging from the ACS Virgo Cluster Survey and found color bimodality in nearly all of the massive S0s in their sample. These S0s fall right on the GC color-galaxy luminosity relations of the Es. If indeed S0s descend from spirals, this is yet another piece of evidence that massive galaxies of all types along the Hubble sequence have very similar GC color distributions, and hence are likely to have experienced similar violent formation processes at some point in their history.

One interesting finding so far confined to S0s was the serendipitous discovery of a new class of star cluster, now known informally as the Faint Fuzzies (FFs). These objects were first detected in the nearby $(10 \mathrm{Mpc})$ S0 NGC 1023. Along with a normal, bimodal system of compact GCs, this galaxy hosts an additional population of faint $\left(M_{V}>-7\right)$ extended $\left(R_{e f f} \sim 7-15 \mathrm{pc}\right)$ star clusters. In deep $H S T$ /WFPC2 images, these objects are confined to an annular distribution closely corresponding to the galaxy's isophotes (Larsen \& Brodie 2000). Spectroscopic follow-up with Keck/LRIS showed that the FFs are metal-rich $([\mathrm{Fe} / \mathrm{H}]$ $\sim-0.5$ ), old $(>8 \mathrm{Gyr}$ ), and rotating in the disk of the galaxy (Brodie \& Larsen 2002). With old ages, inferred masses of $\lesssim 10^{5} M_{\odot}$, and sizes $\sim 5$ times larger than a typical globular or open cluster, these objects occupy a distinct region of age-size-mass parameter space for star clusters. As a population, they have no known analogs in the Milky Way or elsewhere in the Local Group. Similar objects have been found in the S0 galaxy NGC 3384 (Brodie \& Larsen 2002) and NGC 5195, a barred S0 interacting with M51 (Lee, Chandar \& Whitmore 2005; Hwang \& Lee 2006). Peng et al. (2006) found FFs in $\sim 25 \%$ of the S0s in the ACS Virgo Cluster Survey. Due to biases in sample selection, however, this fraction is probably not yet well-constrained. The FFs have relatively low surface brightness, and their properties may be consistent with $M \propto R^{2}$ (unlike GCs, which show no $M-R$ relation). In some cases, their colors are redder than those of the metal-rich GCs in the same galaxy, which may suggest higher metallicities.

Brodie, Burkert, \& Larsen (2004) and Burkert, Brodie \& Larsen (2005) showed 
that the properties of the FFs in NGC 1023 were consistent with having formed in a rotating ring-like structure and explored their origin. Numerical simulations suggest that objects with the sizes and masses of FFs can form inside giant molecular clouds, provided star formation occurs only when a density threshold is exceeded. Such special star forming conditions may be present during specific galaxy-galaxy interactions, in which one galaxy passes close to the center of a disk galaxy, precipitating a ring of star formation. They speculated that the FFs might then be signposts for the transformation of spiral galaxies into lenticulars via such interactions. Alternatively, such conditions might also occur in the inner resonance rings associated with the bars at the centers of disk galaxies. In this case, the old ages of the FFs would suggest that barred disks must have been present at early times.

\section{Spectroscopy}

The detailed properties of individual extragalactic GCs - ages, metallicities, and chemical abundances - are important constraints on theories of GC and galaxy formation. Emphasis has been placed on accurate age-dating of GCs. To the extent that Es formed in recent gas-rich major mergers, this should be reflected in young age measurements for their metal-rich GCs, while both in situ and accretion scenarios predict old ages for both subpopulations.

In principle, integrated-light spectroscopy of individual GCs offers much stronger constraints on ages and elemental abundances than broadband photometry. In practice, even low-resolution spectroscopy is challenging. At the distance of Virgo $(\sim 17 \mathrm{Mpc})$, GCs are already faint, with the turnover of the GCLF occurring at $V \sim 23.5$. Except within the Milky Way and M31, it has so far been possible to assemble only small samples of high-quality GC data, and these have required significant commitments of 8 to $10-\mathrm{m}$ telescope time. Historically, spectroscopic samples have been not only small but also biased to the brightest and/or reddest objects in a given galaxy, forcing a reliance on photometric studies for global conclusions about metallicity distributions. As discussed below, this combination of photometry and small-sample spectroscopy has been used to establish that the ubiquitous color bimodality is due primarily to metallicity differences between the subpopulations, without the need to invoke age differences. There are also biases in spatial sampling: the fields of view of the spectrographs used in most studies are too small to sample the outermost GCs, and the GCs in the central regions are generally undersampled due to the minimum length of slitlets and the high central concentration of most GC systems.

There is a fundamental difference between metallicity and age studies in terms of the conclusions that can be derived from small spectroscopic samples. Age substructure can be identified in biased samples of metal-rich GCs, since younger GCs (with a standard GC mass function) will be brighter than their older counterparts. Thus magnitude-limited samples set an upper limit on the proportion of younger GCs in the system. By contrast, to properly sample the metallicity

distribution, it is necessary to obtain spectra over a color range representative of the entire system. There is no technical reason why large-sample spectroscopic studies with the new generation of highly-multiplexing spectrographs like Keck/DEIMOS and VLT/VIMOS are not feasible, and such work may proliferate in the near future. In Figure 6, we show representative spectra of three 
GCs in M31: an old metal-poor GC, an old metal-rich GC, and an intermediatemetallicity, $\sim 2$ Gyr GC (Beasley et al. 2005).

Most spectroscopic work on extragalactic GCs has utilized Lick/IDS indices (Burstein et al. 1984; Worthey et al. 1994; Trager et al. 1998). These were developed to measure absorption features from $\sim 4000-6400 \AA$ in the spectra of early-type galaxies. Such galaxies typically have large velocity dispersions; thus the low resolution of the Lick system $(\sim 8-11 \AA)$ and wide index bandpasses (tens of $\AA$ ) required by the IDS (Robinson \& Wampler 1972) were not severe impositions. Indices (essentially equivalent widths) are defined in terms of a central bandpass which contains the feature of interest and flanking bandpasses that set the local pseudocontinuum. Observations of distant systems are compared to simple stellar population (SSP) evolutionary models using a set of fitting functions (e.g., Worthey 1994) that predict Lick indices as a function of stellar parameters (e.g., $T_{\text {eff, }} \log g,[\mathrm{Fe} / \mathrm{H}]$ ). Indices measured on modern spectra must be "corrected" to the Lick/IDS system through observations of standard stars.

This system is not optimal for GCs, which have low velocity dispersions and metallicities compared to galaxies. Although the typical $\mathrm{S} / \mathrm{N}$ of extragalactic GC spectra has not justified the use of narrower indices until very recently, the opportunity now exists to improve the placement of index and pseudocontinuum bandpasses and to define new indices in the feature-rich wavelength region below $4000 \AA$. A number of groups are currently defining new index systems on the basis of higher resolution $(\sim 1-3 \AA)$ stellar libraries (e.g., the $2.3 \AA$ MILES library; Sanchez-Blazquez et al. 2006), as well as pursuing the direct fitting of models to observed spectra, avoiding indices entirely (e.g., Wolf et al. 2005).

In the following subsections we discuss observational results on metallicities, ages, and individual elemental abundances of extragalactic GCs.

\subsection{Metallicities and Ages}

Before proceeding, it is worth noting that the term metallicity is not precisely defined in the context of GCs. Some estimates (e.g., composite PCA metallicities; Strader \& Brodie 2004) are tied to Galactic GC metallicities on the Zinn \& West (1984) scale. This scale measures a nonlinear combination of metals and is unlikely to reflect either $[\mathrm{Fe} / \mathrm{H}]$ or "true" $[Z / \mathrm{H}]$. The Carretta \& Gratton (1997) and the Kraft \& Ivans (2004) Fe scales have yet to be extended to the metal-rich regime and calibrations are limited to the metallicity distribution of the Milky Way GC system. This barely touches solar and is poorly matched to the metal-rich regime of GCs in gEs, which extends to solar (and perhaps beyond). The metal-rich GCs in the Galaxy are also few in number and generally highly reddened, as they are concentrated in the bulge. The alternative to direct calibration is to derive metallicities solely from models. A myriad of issues remain with this approach, including uncertain isochrones, meager stellar libraries, corrections to the Lick system, and adjustments for non-solar abundance ratios (see discussion in $§ 4.2$ ). Some authors have adopted the more agnostic $[\mathrm{m} / \mathrm{H}]$ (with "m" for metallicity) to describe their values; others calculate a "corrected" $[\mathrm{Fe} / \mathrm{H}]$ from $[\mathrm{m} / \mathrm{H}]$ or $[Z / \mathrm{H}]$ by subtracting an assumed or derived $[\alpha / \mathrm{Fe}]$ (e.g., Tantalo, Chiosi, \& Bressan 1998). Direct comparisons of metallicities derived from different methods can easily be uncertain at the $\sim 0.2-0.3$ dex level.

One method to estimate metallicities for GCs is to simultaneously use multiple 
lines indices; a weighted combination of six indices was used to derive metallicities for individual GCs in the Milky Way and M87 (Brodie 1981; Brodie \& Hanes 1986). Brodie \& Huchra $(1990,1991)$ applied a refined version of this approach to GCs in a variety of galaxies. Two other studies derived metallicity calibrations using principle components analysis of indices of Galactic GCs: Gregg (1994) used a large set of narrow indices, and Strader \& Brodie (2004) used Galactic GC spectra from Schiavon et al. (2004) to derive a metallicity estimator that is a linear combination of 11 Lick indices. The PCA methods provide accurate metallicities on the Zinn \& West (1984) scale in the range $-1.7 \lesssim[\mathrm{Fe} / \mathrm{H}] \lesssim$ 0.0. The other principal method is to simultaneously derive metallicity and age through comparisons to SSP models (see discussion below).

The bulk of spectroscopic studies of extragalactic GCs have been conducted with two instruments, Keck/LRIS and VLT/FORS. Ages, metallicities, and $[\alpha / \mathrm{Fe}]$ ratios have been estimated predominantly by measuring Lick indices.

In a Keck/LRIS study, Kissler-Patig et al. (1998) inferred that the majority of GCs in the Fornax gE NGC 1399 were old, but that a small percentage might be as young as a few Gyr. However, the robustness of these conclusions was hampered by the low $\mathrm{S} / \mathrm{N}$ of the spectra. In another Keck/LRIS program, Cohen, Blakeslee, \& Ryzhov (1998) studied a sample of $\sim 150$ GCs in M87. Despite large errors on the absorption line indices measured in many of their spectra, the size of the sample allowed a good statistical comparison with spectra of Milky Way GCs. The M87 and Milky Way GCs were found to populate similar areas in Mgb vs. $\mathrm{H} \beta$ and $\mathrm{Mg} b$ vs. Fe5270 diagrams, suggesting that the M87 GCs have ages and $[\mathrm{Mg} / \mathrm{Fe}]$ ratios comparable to those of the Galactic GCs. These ages and $[\alpha / \mathrm{Fe}]$ ratios are known, from detailed analysis of individual cluster stars, to be $\gtrsim 10$ Gyr and $\sim+0.2-0.3$, respectively (e.g., Carney 2001). Beasley et al. (2000) found old ages for coadded (on the basis of $C-T_{1}$ color) WHT spectra of GCs in NGC 4472. Kuntschner et al. (2002) presented a study of the S0 NGC 3115 showing that GCs in both the metal-poor and metal-rich subpopulations are old.

Subsequent studies undertaken with Keck/LRIS include: Ellipticals - NGC 1399 (Forbes et al. 2001); NGC 4472 (Cohen, Blakeslee, \& Côté 2003); NGC 4365 (Larsen et al. 2003; Brodie et al. 2005); NGC 3610 (Strader et al. 2003; Strader, Brodie, \& Forbes 2004b); NGC 1052 (Pierce et al. 2005); NGC 1407 (Cenarro et al. 2006); S0s-NGC 1023 (Brodie \& Larsen 2002); NGC 524 (Beasley et al. 2004); Spirals-M81 (Schroder et al. 2002); NGC 4594 (Larsen et al. 2002). In all cases it was concluded that at most a small fraction of the observed samples of GCs were young or of intermediate age $(\lesssim 5-6 \mathrm{Gyr})$. In the case studies of the intermediate-age merger remnant E NGC 3610, Strader et al. (2003, 2004b) found that only two out of ten prime candidate intermediate-age GCs had ages consistent with formation in the merger. Figure 7 shows a typical index-index plot used to derive metallicities and ages for GCs, in this case, from NGC 1407 (Cenarro et al. 2006). Here the GCs studied appear to have ages consistent with Galactic GCs, but extend to higher metallicities.

In an effort to use more of the information available in a spectrum than is contained in a traditional index-index plot, Proctor, Forbes, \& Beasley (2004; see also Proctor \& Sansom 2002) developed a $\chi^{2}$ minimization routine that simultaneously fit all of the Lick indices to SSP models, exploiting the different sensitivities of each of the indices to age, metallicity, and $[\alpha / \mathrm{Fe}]$. Metallicities derived in this manner are complementary to ones derived using the PCA methods described above, and, for GCs in the range over which the PCA metallicities 
are calibrated, the agreement is excellent (e.g., Cenarro et al. 2006). Multi-index approaches offer the advantage of minimizing random and systematic problems in any individual index, such as may arise in low $\mathrm{S} / \mathrm{N}$ data. Moreover, where the GC background is an emission line region (from either sky or the host galaxy), or when individual element abundance anomalies or extreme horizontal branch morphologies may be present in the GC, such precautions are essential (see below).

The best of the Keck data were compiled in a meta-analysis by Strader et al. (2005). This work synthesized more than a decade's worth of effort on the GCs systems of a heterogeneous sample of galaxies, spanning the range from dwarfs to gEs (all but NGC 4594 were of early-type). A typical galaxy had only 10-20 spectra of sufficiently high $\mathrm{S} / \mathrm{N}$ to be included in the analysis. Lick indices measured from these spectra were combined, and ages were derived through direct differential comparison to indices of Galactic GCs, in part to avoid SSP model uncertainties. This study showed that both the metal-poor and metal-rich GC subpopulations had mean ages as old as (or older than) Galactic GCs. In Figure 8, from Strader et al. (2005), PCA metallicity is plotted against a composite Balmer line index of $\mathrm{H} \beta$ and $\mathrm{H} \delta_{A}(\mathrm{H} \gamma$ was excluded because the strong $\mathrm{G}$ band lies in its blue pseudocontinuum bandpass). The implication from their sample of galaxies is that GC formation, and by extrapolation, the bulk of star formation in spheroids, took place at early times $(z \gtrsim 2)$. As discussed in the Annual Review of Renzini (2006, this volume), fossil evidence from the local universe and in situ studies at high redshift lead to similar conclusions: most of the stars in massive Es formed at $z \sim 2-5$. Of course, studies of larger samples of GCs in a wide range of galaxies are needed to further test this result.

This conclusion is still consistent with the existence of young early-type galaxies in the local universe (Trager et al. 2000), because galaxy age estimates are luminosity-weighted. A small "frosting" of recent star formation can easily result in the measurement of young spectroscopic ages for galaxies whose stars are predominately old, and there is an inverse relationship between the age and required burst strength. In addition, radial age gradients in early-type galaxies are often measured. Younger ages are derived from spectra extracted from small (1/8 of the effective radius; $\left.r_{e}\right)$ apertures than from larger $\left(r_{e} / 2\right)$ ones. In any case, a small amount of star formation is expected to produce a correspondingly small subpopulation of young GCs, which could easily have escaped detection in existing samples. High quality spectra of $>100$ GCs per galaxy, now obtainable with highly multiplexing instruments such as Keck/DEIMOS and VLT/VIMOS, would be necessary to probe these low-level star-forming events.

Puzia et al. (2005; see also Puzia 2003) presented a spectroscopic analysis of a more homogeneous sample of galaxies, comprising 5 Es and 2 S0s, all with $-19.2>M_{B}>-21.2$, and mostly in small groups. The low end of this luminosity range is near $L^{*}$, so their sample, like that of Strader et al. (2005), was dominated by early-type galaxies brighter than the knee of the galaxy LF. They included a detailed discussion of the advantages of the different Balmer lines in terms of dynamic range, age-metallicity degeneracy, and ability to correct to the Lick system. Using a combined Balmer line index and the $\alpha$-insensitive metallicity proxy [MgFe]' (Thomas, Maraston, \& Bender 2003), Puzia et al. found 4 GCs (out of their total of 17 high-quality spectra) with index measurements consistent with ages less than 9 Gyr (their Figure 7 ). While this sample is not sufficient to address the proportion of young GCs that might be present in any individual 
galaxy, the presence of some younger GCs in these typically group galaxies would be consistent with "downsizing", whereby lower-mass galaxies in lower-density environments tend to have younger mean ages (Cowie et al. 1996). There remains disagreement on the proportion of young GCs present in galaxies of moderate luminosity.

The evidence that most GCs in massive galaxies are quite old is relevant to the distinction between "core" and "power-law" E galaxies seen in the local universe. These designations stem from the surface brightness profiles in the very inner parts of the galaxy. The core galaxies tend to be bright $\left(M_{V} \lesssim-21.5\right)$, have boxy isophotes, and do not rotate; the power-law galaxies are fainter, have disky isophotes, and are at least partially rotationally supported (Kormendy \& Bender 1996; Faber et al. 1997). The working hypothesis for the creation of central cores is scouring by merging binary black holes. Kormendy et al. (2006) have unified this scenario by demonstrating that power-law galaxies have excess light in their central parts, above an extrapolation of a best-fit Sersic profile in the outer parts. Core galaxies have no such excess light. They argue that the distinction between core and power-law galaxies represents Es whose last major merger was dry or wet, respectively (i.e., without or with star formation). In power-law galaxies, black hole scouring may still have occurred, but it is swamped by the light from the stars in the center that formed in the merger. The old GC ages inferred from observations tentatively indicate that, even in power-law galaxies, the wet merger that formed the E either occurred long ago, or produced only a relatively small amount $(\lesssim 10-20 \%)$ of the galaxy's current stellar mass. However, much larger spectroscopic samples of GCs in power-law galaxies are needed to better constrain the fraction of mass produced in wet mergers.

4.1.1 UNCERTAINTIES An important uncertainty in age determinations is the level of contribution from hot stars to the integrated spectra of GCs. These can "artificially" enhance the Balmer line strengths beyond the values set by the main sequence turnoff and can lead to an underestimation of the GC age. Blue stragglers and blue horizontal branch stars are the primary offenders-hotter stars, including extreme HB, AGB-manque, and post-AGB stars have little flux in the optical region where ages are usually derived. At least in the Milky Way, blue stragglers have quite a small effect on a GC's optical integrated spectrum (e.g., Schiavon et al. 2002). It is worth noting that this may not necessarily be the case in extragalactic GCs that have high binary fractions or are very compact.

This leaves blue HBs stars as the main systematic source of uncertainty in GC age estimates. HB morphologies are primarily set by GC metallicity, with Balmer line strengths peaking at $[\mathrm{Fe} / \mathrm{H}] \sim-1.3$ and decreasing toward lower metallicities as the stars become hotter and $\mathrm{H}$ is ionized. The classic "secondparameter problem" - that GCs of a given metallicity can have quite different HB morphologies - is currently unresolved, despite intensive research efforts over several decades. It has frequently been suggested that age is the second parameter (e.g., Chaboyer, Demarque, \& Sarajedini 1996). However, this does not appear to be true in the few cases where direct turnoff age comparisons can be made by normalizing the main sequence luminosity functions (e.g, Stetson, Vandenbergh, \& Bolte 1996). While age differences certainly will produce changes in HB morphology, it is dangerous to use the $\mathrm{HB}$ as an age indicator. As an example, several metal-poor GCs in M33 were studied with $H S T$ /WPFC2 by Sarajedini et al. (2000) and found to have unusually red HBs for their metallicities. Sara- 
jedini et al. suggested intermediate ages might be the cause. However, Larsen et al. (2002) derived dynamical masses for these GCs and instead found that they had M/L ratios typical of old GCs. At present there is no known a priori predictor of GC HB morphology. Consequently, various HB diagnostics have been suggested. These include (i) the $\mathrm{Ca} \mathrm{H}+\mathrm{K}$ line strength inversion; enhanced $\mathrm{H} \epsilon$ from hot stars can increase the $\mathrm{Ca} \mathrm{H}$ line equivalent width compared to the $\mathrm{Ca} \mathrm{K}$ line (Rose 1985), (ii) Near-ultraviolet $(N U V-V)$ colors, and (iii) a tendency for progressively younger ages to be inferred from increasingly higher-order Balmer lines because of an increasing contribution from putative blue HB stars (Schiavon et al. 2004). The effect of BHB stars on integrated Balmer line strength can be substantial: Puzia et al. (2005) estimated that $H \beta$ may be increased by up to 0.4 $\AA$ (based on their analysis of Galactic GCs), while Maraston (2005) inferred an even greater effect from her SSP models at high metallicities (see also Thomas, Maraston, \& Korn 2004).

Aside from the classic second-parameter effect, there is some evidence that the HB morphologies of GCs in other galaxies can be quite different from those in the Milky Way. For example, in a HST/WFPC2 study by Rich et al. (2005), a sample of 12 M31 GCs appeared offset from the Milky Way HB-metallicity relation. One potential explanation is that these M31 GCs are $\sim 1-2 \mathrm{Gyr}$ younger than their Galactic counterparts. More startling is the finding by Sohn et al. (2005), from an HST/STIS FUV imaging study of bright M87 GCs, that all the GCs have much bluer $F U V-V$ colors than Galactic GCs of the same metallicity. Increasing the age of the M87 GCs relative to Galactic GCs is one potential solution, but it would require implausibly large age differences of 2-4 Gyr to bring the two samples into line. Fuel consumption arguments, and the fact that spectroscopic ages of these GCs are old (Cohen et al. 1998), suggest that the hot stars in M87 GCs are a redistribution of stars blueward of a normal $\mathrm{BHB}$, and not simply an extension of it. A caveat to this finding is the UV-flux limited nature of their sample, which tends to select the most extreme GCs.

A night of 8-10-m telescope time can generate spectra of GCs at the distance of Virgo $(\sim 17 \mathrm{Mpc})$ from which $\mathrm{H} \beta$ line index strengths can be measured to an accuracy of $\sim 0.1 \AA$ for GCs with $V \sim 21.5$ (for fainter GCs, the errors are correspondingly larger). As Figure 7 illustrates, this translates into an error of $\sim 1-2$ Gyr at old ages. Since the isochrones are more widely separated at younger ages, the ability to discriminate fine age differences improves with decreasing age. In these SSP model grids, BHB effects actually cause the isochrones to cross at low metallicities and old ages, setting a fundamental upper limit on our age estimates of $\sim 10 \mathrm{Gyr}$ at these metallicities. It is important to remember that the actual ages of the majority of GCs in the universe could well be older than this.

\section{$4.2[\alpha / \mathbf{F e}]$}

It is widely recognized that measurements of the $[\alpha / \mathrm{Fe}]$ ratio of a stellar population can provide valuable insight into its star formation history, particularly by constraining the timing and duration of a starburst. Most $\alpha$-elements (e.g., $\mathrm{Mg}$, $\mathrm{Ti}, \mathrm{Ca}, \mathrm{Si}$ ) and $1 / 3$ of the $\mathrm{Fe}$ (for the solar mixture) are generally thought to form in Type II SNe. Since these come from the explosions of massive stars, they closely trace the star formation rate, and begin to ignite within tens of Myr of the onset of a starburst. The remainder of the Fe is produced in Type Ia SNe, which 
typically occur over Gyr timescales. Enhanced $[\alpha / \mathrm{Fe}]$ ratios would thus indicate rapid star formation, occurring before there was time for significant enrichment of the interstellar medium by Type Ia SNe. This is generally the case in earlytype galaxies (e.g., Worthey, Faber, \& Gonzalez 1992; Matteucci 1994; Trager et al. 2000; Thomas et al. 2005). A complication is that the timescale over which fresh ejecta become incorporated into interstellar gas is not necessarily known. Other nucleosynthetic sites, operating on a variety of time scales, also contribute to the cosmic mix (e.g., AGB stars produce a significant amount of s-process and light elements).

The naive expectation for metal-poor GCs in external galaxies is that they will have supersolar $[\alpha / \mathrm{Fe}]$, since they formed early in the universe, before there was substantial metal enrichment. The situation for metal-rich GCs is less clear, as it will depend upon the exact formation mechanism. If they formed along with most of the field stars in massive Es, they should share the $[\alpha / \mathrm{Fe}] \sim+0.2-0.4$ of their parent galaxies. They could perhaps reach even higher values if they formed preferentially early in the starburst. Metal-rich GCs formed at lower redshift from $\sim$ solar metallicity gas in major disk-disk mergers might be expected to have $[\alpha / \mathrm{Fe}]$ closer to 0 , since it is more difficult to enhance $[\alpha / \mathrm{Fe}]$ when starting from a high metallicity (although metallicity gradients in spiral disks must also be considered). The results from extragalactic GCs do not generally agree with these expectations, though much of this may be due to the observational and technical difficulties in accurately estimating $[\alpha / \mathrm{Fe}]$, as discussed below.

In theory, it should be very easy to determine $[\alpha / \mathrm{Fe}]$ from low-resolution $\mathrm{GC}$ spectra. $\mathrm{Mg}$ is the element of choice because of the strong $\mathrm{Mg} b$ triplet and $\mathrm{MgH}$ bandhead in the optical. Trager et al. (2000) described a method for estimating $[\mathrm{Mg} / \mathrm{Fe}]$ using models by Worthey (1994) and corrections from Trippico \& Bell (1995). However, the widespread estimation of this quantity in extragalactic GCs did not occur until Thomas, Maraston \& Bender (2003) published the first models to explicitly include the effects of $\alpha$-enhancement.

In practice, the interpretation of the observations using these models has been complicated. The GCs in the metal-rich subpopulations in a variety of massive galaxies have been found to have $[\alpha / \mathrm{Fe}]$ ranging from 0 to $\sim+0.3$ (e.g., Kuntschner et al. 2002; Beasley et al. 2005) or higher (Puzia et al. 2005 find $\sim+0.45$ but with large scatter). By contrast, the metal-poor GCs frequently appear to have $[\alpha / \mathrm{Fe}] \sim 0$ or even lower (e.g., Olsen et al. 2004; Pierce et al. 2005), although the convergence of SSP model lines at low metallicities increases the errors on these determinations of $[\alpha / \mathrm{Fe}]$. If $[\alpha / \mathrm{Fe}]$ for metal-poor $\mathrm{GCs}$ were indeed this low, it would be quite surprising. In the simple view of nucleosynthesis outlined above, supersolar $[\alpha / \mathrm{Fe}]$ results from star formation over "short" $(<1 \mathrm{Gyr})$ timescales while solar or subsolar $[\alpha / \mathrm{Fe}]$ indicates very extended star formation histories. Thus we expect the metal-poor GCs to have high $[\alpha / \mathrm{Fe}]$.

Galactic GCs from both subpopulations have $[\alpha / \mathrm{Fe}] \sim+0.3$ (e.g., Carney 1996) or perhaps a little lower for some metal-rich bulge GCs. These values are quite closely reproduced by the popular Thomas et al. (2003) SSP models for the Galactic GC data of Puzia et al. (2002), although the results from applications to extragalactic GCs are mixed (as described above). Current stellar libraries and the Worthey (1994) fitting functions utilize few stars with metallicities in the range of metal-poor GCs, and SSP models may be more uncertain in this regime.

Schiavon (2006) gave a good summary of the issues involved in creating non- 
solar $[\alpha / \mathrm{Fe}]$ models. These include (i) the importance of employing the proper isochrones and luminosity functions, and (ii) the need to correct the index predictions of the fitting functions. Item (ii) is usually carried out differentially, using stellar models for stars in representative parts of the CMD. A preliminary attack on this problem by Trippico \& Bell (1995), using just three stars, produced broadly similar results to the more thorough treatment of Korn et al. (2005). Korn et al. also discussed in detail the elemental sensitivities of the individual Lick indices.

A potentially confusing difference among models is the treatment of $[\alpha / \mathrm{Fe}]$ vs. $[\mathrm{Fe} / \mathrm{H}]$. Thomas et al. (2003) calculated their models at fixed total metallicity $([Z / \mathrm{H}])$ and produced supersolar $[\alpha / \mathrm{Fe}]$ by lowering $[\mathrm{Fe} / \mathrm{H}]$, since the dominant component of $Z$ is the $\alpha$-element O. By contrast, Schiavon (2006) calculated models at fixed $[\mathrm{Fe} / \mathrm{H}]$. Schiavon's approach has the benefit of a direct link to the measurable quantity $[\mathrm{Fe} / \mathrm{H}]$.

These SSP uncertainties, when combined with the wide Lick index bandpasses, which always admit contributions from other elements in addition to the targeted feature, make most current estimates of $[\alpha / \mathrm{Fe}]$ untrustworthy. We suggest intensive study of nearby GC systems (e.g., M31), where potentially more accurate results from high-resolution integrated spectroscopy of GCs can be directly compared to low-resolution spectra.

\subsection{Abundance Anomalies}

In addition to the $\alpha$-elements, GCs show a variety of abundance anomalies with respect to the solar mixture. This is most clear in the Galaxy, where detailed study of individual stars in GCs is possible (see the Annual Review of Gratton, Sneden, \& Carretta 2004). In extragalactic GCs, the most obvious anomaly is CN-enhancement. This was first reported in M31 GCs by Burstein et al. (1984), and, at least in this galaxy, appears to be due to an excess of $\mathrm{N}$ above even the levels in Galactic GCs (see §6.1), which themselves are enhanced in N over the solar mixture. Trager (2004) has argued that a similar CN anomaly (presumably due to N) is present in many GC systems, including NGC 3115 (Kuntschner et al. 2002), the old GCs in NGC 3610 (Strader et al. 2003a), and the Fornax dSph (Strader et al. 2003b). It is also present in GCs in the gE NGC 1407 (Cenarro et al. 2006). We must consider very high $\mathrm{N}$ abundances to be a generic feature of the early chemical evolution of GC systems (and perhaps their host galaxies). Interestingly, of the two confirmed metal-rich intermediate-age GCs in NGC 3610, one shows the $\mathrm{CN}$ anomaly, and one does not.

\section{$5 \quad$ Near-IR Imaging}

A new tool for studying GCs emerged during the last few years with the advent of large-format infrared detectors. Kissler-Patig (2000) pointed out that it should be possible to use near-IR (NIR) photometry of GCs to break the age-metallicity degeneracy inherent in optical colors. The $V-K$ color of an old GC is a measure of the temperature of its red giant branch. This temperature is strongly dependent on metallicity but has little sensitivity to age. The prospect of large NIR imaging surveys is exciting because it offers the potential to accurately measure individual ages and mean metallicities for large numbers of GCs in galaxies out to the distance of the Virgo cluster and beyond. 
Kissler-Patig and collaborators, primarily using VLT/ISAAC, have taken the lead in such NIR studies. Puzia et al. (2002) reported a comparison in VIK between the GC systems of the group S0 NGC 3115 and the Virgo E NGC 4365. The GC system of NGC 3115 appeared bimodal in color-color space, while the color distribution of GCs in NGC 4365 looked largely unimodal, with a formal peak at supersolar metallicity and intermediate age $(\sim 2-5 \mathrm{Gyr})$. However, the presence of a large subpopulation of intermediate-age GCs was confusing, as the central light of the galaxy itself is uniformly old (Davies et al. 2001). How could a large number of GCs form without an accompanying field star component? This puzzling result precipitated a flurry of activity on the GC system of NGC 4365. Spectroscopy of a subset of the candidate intermediate-age GCs seemed to confirm their young ages, while demonstrating that other young candidates (selected from their optical/NIR colors) were in fact old (Larsen et al. 2003).

However, follow-up spectroscopy with somewhat higher $\mathrm{S} / \mathrm{N}$ found no evidence for any intermediate-age clusters. Moreover, when considered in combination with new HST /ACS imaging, these data seemed to point instead to three subpopulations of old GCs, with a very centrally-concentrated, intermediate-metallicity subpopulation filling in the gap between the normal metal-poor and metal-rich GCs (Brodie et al. 2005). A similar intermediate-metallicity subpopulation has been discovered by Beasley et al. (2006) in the gE NGC 5128. This interpretation is consistent with wider-field $K$-band photometry obtained by Larsen, Brodie, \& Strader (2005). These authors discussed all of the available data and the details of the error estimates, and showed evidence for systematic errors in optical-NIR SSP models. Nonetheless, based on a comparison of HST/NICMOS $H$-band photometry of 70 GCs in NGC 4365 and 11 GCs NGC 1399 with SSP models, Kundu et al. (2005) concluded that a large number of intermediate-age (2-8 Gyr) GCs with metallicities up to $[\mathrm{Fe} / \mathrm{H}]=+0.4$ are present in NGC 4365 . Young metal-poor GCs would also be inferred from the distribution of their data on SSP model grids. Since metal-poor GCs are expected to be old under nearly all formation scenarios, this further suggests that at least some of the offset of metal-rich GCs to young ages may be due to systematic errors in the SSP models (Larsen et al. 2005). Clearly, the last word has not yet been spoken on this intriguing galaxy.

Hempel et al. (2003) studied the early-type galaxies NGC 5846 and NGC 7192 with NIR photometry, reporting that the former galaxy hosts a large population of intermediate-age GCs. Followup $U$-band photometry in NGC 5846 and NGC 4365 (Hempel \& Kissler-Patig 2004) was argued to support the earlier results, although the number of GCs detected was low. Follow-up spectroscopy does not appear to support the presence of a large subpopulation of intermediate-age GCs in NGC 5846 (Puzia et al. 2005). The fraction of GCs with ages formally $<8$ Gyr in the observed sample of luminous GCs is $15-20 \%$ (T. Puzia, private communication).

Goudfrooij et al. (2001a) presented the best evidence to date of a significant population of intermediate-age GCs in any post-merger galaxy. They used a combination of HST and ground-based optical and NIR photometry of GCs in NGC 1316, a merger remnant E in the Fornax cluster. They found metal-rich GCs that were substantially brighter than normal GCs at that distance and deduced photometric ages of $\sim 3$ Gyr for these bright red objects. These photometric ages were confirmed by spectroscopy of a small number of the brightest clusters (Goudfrooij et al. 2001b). However, even in this galaxy, the strongest pieces of 
evidence for an intermediate-age subpopulation are (i) the presence of unusually luminous GCs and (ii) the power-law (rather than log-normal) GC luminosity function of the metal-rich GCs (see also Goudfrooij et al. 2004).

Improvements in NIR SSP models will brighten the prospects for future NIR studies and help this field to reach its full potential. In addition, the launch of the James Webb Space Telescope (JWST) in the next decade will allow the collection of high-quality $K$-band photometry for many GC systems.

\section{Globular Cluster-Field Star Connections}

Other than the Milky Way, the GC systems of only two massive galaxies have been observed in detail, in the sense that a significant number of their GCs have been observed with high $\mathrm{S} / \mathrm{N}$ spectroscopy and we can study their field stars directly. M31, our sister spiral galaxy in the Local Group, and NGC 5128, one of the nearest massive E galaxies, are both sufficiently well-studied that they can elucidate the role of GCs in tracing star formation in their parent galaxies.

\subsection{M31}

Though the total mass of M31 is similar to that of the Galaxy (Evans \& Wilkinson 2000 ), it has a GC system $\sim 3$ times as large, with $\sim 400-450$ GCs (van den Bergh 1999; Barmby \& Huchra 2001). A comprehensive catalog of photometric and spectroscopic data for M31 GCs was assembled by Barmby et al. (2000) and used to show that, broadly speaking, the M31 GC system is quite similar to that of the Galaxy; M31 has an extended halo subpopulation and a more centrally-concentrated rotating bulge/disk subpopulation.

The kinematics of the M31 GC system may be complex. Perrett et al. (2002) found that the metal-rich GCs have a velocity dispersion similar to the bulge $(\sim 150 \mathrm{~km} / \mathrm{s})$ and a rotational velocity of $160 \mathrm{~km} / \mathrm{s}$. Surprisingly, they also found a large rotational velocity for the metal-poor GCs, $\sim 130 \mathrm{~km} / \mathrm{s}$. By contrast, the metal-poor GCs in the Galaxy show little evidence for rotation. Visually, the spatial distribution of the Perrett et al. metal-poor GC sample appears to be the superposition of a centrally-concentrated spherical population and a relatively thin disk component. Indeed, Morrison et al. (2004) used the Perrett et al. velocities to argue that M31 possesses a rapidly rotating "thin disk" of old metal-poor GCs. Beasley et al. (2004) presented high-S/N spectra of a small number of these putative old metal-poor GCs and showed that they have spectra more similar to young $(<1 \mathrm{Gyr})$, approximately solar metallicity objects. Their luminosities suggest stellar masses comparable to massive Galactic open clusters or low-mass GCs. The presence of young GCs has also been claimed by Burstein et al. (2004) and Puzia, Perrett, \& Bridges (2005) (both based in part on data from Beasley et al. 2004) and by Fusi-Pecci et al. (2005). Cohen et al. (2005) used Keck NIR/AO imaging to show that four of these very young clusters are actually "asterisms" - either chance groupings of bright stars or a few brighter stars superimposed upon sparse open clusters. A more detailed examination of archival $H S T$ imaging indicates that perhaps half of the candidate young clusters are real; previous HST/WFPC2 had confirmed the existence of four such clusters (Williams \& Hodge 2001). A high-resolution imaging survey of the disk will be needed to assess the true number of young GCs. In any case, if these interloping objects (asterisms and/or YMCs) are removed from the metal-poor GC candidate 
list, much of the rotational signature observed by Perrett et al. would be erased. The apparent lack of an old metal-poor thin disk of GCs is important, since the existence of this disk would rule out any significant merger having taken place in the last $\sim 10$ Gyr. As discussed below, such a merger is the working theory for the existence of intermediate-age stars in M31's halo.

Due to selection effects, including reddening in the inner disk and the paucity of GC candidates at large radii, the overall fraction of metal-poor to metal-rich GCs is still poorly constrained in M31. From their kinematic and metallicity study, Perrett et al. (2002) found that only $\sim 25 \%$ of a sample of $\sim 300$ GCs belong to the metal-rich (bulge) peak, compared to one third in the Galaxy. If, as discussed above, many of the candidate metal-poor GCs in M31 are interlopers, then the ratio of metal-poor to metal-rich GCs in M31 could be quite consistent with that found for the Galaxy (2:1). There are several ongoing studies of the M31 GC system with MMT/Hectospec that should provide a clearer picture of this situation.

Burstein et al. (1984) found evidence for Balmer line anomalies in M31 GCs compared to GCs in the Milky Way. Their preferred explanation was that the M31 GCs might be younger than their Milky Way counterparts. Brodie \& Huchra (1991) and Beasley et al. (2004) compared integrated-light spectra of Milky Way and M31 GCs at the same metallicity and found no evidence for enhanced Balmer lines in M31 GCs. Beasley et al. (2005) extended these results by fitting the Lick indices measured from their sample GCs to stellar population models. They employed the multi-index $\chi^{2}$ minimization method developed by Proctor, Forbes, \& Beasley (2004) to derive ages and metallicities for these clusters. In addition to the normal old metal-poor and metal-rich GC subpopulations, they found a group of six GCs with intermediate metallicities and ages of 3-6 Gyr. A larger number of GCs with similar ages were also reported by Puzia et al. (2005). This group of GCs forms a coherent chemical and kinematic group of objects in M31, consistent with the accretion of an SMC-type galaxy within the last several Gyr (in the Galaxy, there are several several comparable GCs associated with the Sgr and CMaj dSphs; §10). Ashman \& Bird (1993) had previously analyzed M31 GC kinematics and found evidence for $\sim 7$ distinct groups of GCs. The clustering analysis of Perrett et al. (2003) found $\sim 8$ such groups, but Monte Carlo simulations indicated that statistically half or more of these should be chance groupings. In an addition to the rapidly growing zoo of stellar clusters, Huxor et al. (2005) have identified 3 metal-poor objects in the halo of M31 that have luminosities typical of GCs, but sizes from 25-35 pc. They suggest that these could be stripped nuclei of dwarfs, analogous to $\omega$ Cen in the Galaxy (Majewski et al. 2000).

Brown et al. (2004) obtained extremely deep HST / ACS photometry of the M31 GC 379-312 ([Fe/H -0.6) and through isochrone fitting derived a formal age of $10_{-1}^{+2.5} \mathrm{Gyr}$, perhaps $\sim 1$ Gyr younger than Galactic metal-rich GCs. Rich et al. (2005) carried out photometry deeper than the horizontal branch for a sample of 12 M31 GCs. They found the classic second parameter effect, but with an offset that might be explained if the GCs were $\sim 1-2$ Gyr younger than their Galactic counterparts. These results hint at an interesting relative age difference between the two GC systems, but hard evidence will be difficult to come by. It would require a UV/optical space telescope with an aperture larger than $H S T$ to derive turnoff ages for significant numbers of M31 GCs.

Burstein et al. (1984) also discovered CN enhancements in M31 GCs (with 
respect to Galactic GCs). This result was later confirmed by Brodie \& Huchra (1991), who pointed out that CH at $4300 \AA$ did not appear to be anomalous, and argued that the observed $\mathrm{CN}$ enhancement might be due to an overabundance of N. Beasley et al. (2004) did not see the enhancement clearly in the Lick $\mathrm{CN}_{2}$ index, which measures the weaker $4215 \AA$ bandhead, but it is obvious in a different index sensitive to the $3883 \AA$ transition. That $\mathrm{N}$ is the culprit in $\mathrm{CN}$ variations was confirmed directly by near-UV spectra of Galactic and M31 GCs around the $\mathrm{NH}$ band at $3360 \AA$ (Burstein et al. 2004). Observations in this region of the spectrum have been limited by the low fluxes of GCs and the low UV efficiencies of telescopes, instruments, and detectors. As a result, the SSP modeling effort has proceeded slowly, and quantitative $[\mathrm{N} / \mathrm{Fe}]$ values do not yet exist. Thomas, Maraston, \& Bender (2003) found that $[\mathrm{N} / \mathrm{Fe}] \sim+0.8$ was required to match CN in some Galactic GCs; in M31 GCs it must be even higher. Since NH (in the UV) is dominated by light from stars around the main sequence turnoff, the $\mathrm{N}$ enhancements are probably primordial in origin and cannot be attributed to non-canonical mixing processes on the red giant branch.

M31's stellar halo, studied in fields from $\sim 10-30$ projected kpc, appears predominately metal-rich $([\mathrm{m} / \mathrm{H}] \sim-0.5)$, a full 1 dex higher than the Milky Way (e.g., Mould \& Kristian 1986; Durrell et al. 1994). In one M31 halo field, Brown et al. (2003) found that $\sim 30 \%$ of the stars were of intermediate-age, again unlike the Milky Way. Given the large disk and bulge of M31, it has been suggested that these results may be explained by the contamination of putative halo samples by disk/bulge stars (Worthey et al. 2005). However, this interpretation does not appear to be consistent with recent kinematic studies of a subset of the intermediate-age stars (Rich et al. 2006). In any case, M31 also appears to have a metal-poor projected $r^{-2}$ stellar halo, like the Galaxy (Guhathakurta et al. 2005; Kalirai et al. 2006). Numerical simulations of M31 indicate that a relatively minor accretion event (involving an LMC-sized galaxy) would suffice to redistribute the observed number of metal-rich, intermediateaged stars from the disk into the halo (Font et al. 2006).

Before discussing how field star properties relate to GCs, it is helpful to define the quantity $T^{n}$, which is the Zepf \& Ashman (1993) $T$ parameter normalized to the mass of a particular stellar population (vs. the original definition, which normalizes a single GC subpopulation to the total stellar mass in the galaxy). For spheroids with few (or no) metal-poor stars, $T_{\text {red }}^{n}$ is the same as $T_{\text {red }}$, modulo uncertainties in the adopted $M / L$.

The Galaxy has $\sim 100$ metal-poor GCs and a halo mass of $\sim 10^{9} M_{\odot}$ (Carney, Latham, \& Laird 1990), resulting in a metal-poor $T_{b l u e}^{n} \sim 100$. By contrast, the corresponding metal-rich value is $T_{\text {red }}^{n} \sim 5$ (there are only $\sim 50$ metal-rich GCs and bulge mass $\sim 10^{10} M_{\odot} ;$ Kent 1992). Thus the metal-poor GCs formed with an efficiency twenty times higher than the metal-rich GCs with respect to field stars.

In the Galaxy, the association of the metal-poor GCs with halo stars is clear; they have similar peak metallicities, spatial distributions, and kinematics (though the metallicity dispersion of the field stars is much larger than that of the GCs). The situation for the metal-rich GCs is somewhat less well-defined but, in general, their spatial distribution and kinematics are more similar to the bulge than the thick disk (see $§ 7.2$ ). However, the metallicity distribution of the metal-rich GCs does not match that of the bulge. Fulbright et al. (2006) used Keck/HIRES spectra to recalibrate the metallicity distribution of red clump stars in Baade's 
Window (Sadler et al. 1996). They found a peak at $[\mathrm{Fe} / \mathrm{H}] \sim-0.15$, roughly $0.3-0.4$ dex higher than the peak of the metal-rich GCs. Baade's Window is located beyond one bulge effective radius, so if the bulge has a negative metallicity gradient the mean offset could be even larger.

The metallicity of the M31 bulge is similar to the bulge of the Galaxy (Sarajedini \& Jablonka 2005). The GC color distributions in the Milky Way and M31 show no significant differences, suggesting a similar metallicity offset in M31 between the metal-rich GCs and the bulge (as borne out by spectroscopy of individual GCs in M31). The metallicity of the metal-poor component of the stellar halo in M31 is not well-constrained, so no direct comparison can yet be made with the GCs.

\subsection{NGC 5128}

As one of the nearest (albeit disturbed) giant ellipticals ( 4 Mpc), NGC 5128 holds special significance for stellar population studies. W. and G. Harris and coworkers have taken the lead in studying the relationship between GCs and field stars in this galaxy.

Their HST/WFPC2 photometry of red giants in fields from $\sim 8$ to $31 \mathrm{kpc}$ (projected radius) give metallicity distributions that peak at $[\mathrm{m} / \mathrm{H}] \sim-0.2$ to $\sim-0.4$, with a slight negative metallicity gradient with galactocentric radius (Durrell, Harris \& Pritchet 2001; Harris \& Harris 2002). Such metallicities are consistent with those of the metal-rich GCs, modulo uncertainties in the relative zeropoints of the metallicity scales. However, the presence of a radial metallicity gradient in the field stars complicates any comparison with the flat metallicity distribution of the GCs. At what radius should the two be compared? The field star metallicity distribution is reasonably well-fit with an accreting box model like the one needed to solve the Galactic G-dwarf problem (Lynden-Bell 1975). Rejkuba et al. (2005) used an HST/ACS outer halo (38 kpc) pointing to derive a metallicity distribution for NGC 5128 field stars very similar to that of Harris et al., except that they found a larger number of metal-rich stars ( These stars were missed by Harris et al. because of large bolometric corrections in $V-I$ at high metallicities. An age analysis of the Rejkuba et al. field, based primarily on the locations of the ABG bump and the red clump, yielded a mean age of $\sim 8$ Gyr. However, the data are also consistent with a two-component model, comprising an older base population plus a small percentage of 5-7 Gyr stars. Based on the number of AGB stars (Soria et al. 1996) and Mira variables (Rejkuba et al. 2003), a figure of $\sim 10 \%$ has been suggested for the fraction of the total stellar mass in intermediate-age stars. This is consistent with the $7 \%$ fraction of intermediate-age GCs in the galaxy (see below).

NGC 5128 possesses only a relatively minor tail of metal-poor "halo" stars, even in the outermost pointing, but (as in M31) it is unclear whether this offers a constraint on the presence or absence of a Milky Way-like metal-poor projected $\sim r^{-2}$ halo. Harris \& Harris (2002) inferred from the paucity of metal-poor stars that $T_{b l u e}^{n}$ must be much larger than $T_{\text {red }}^{n}$. They argued for a scenario in which the metal-poor GCs formed at the beginning of starbursts in small potential wells. These could be efficiently evacuated by supernova feedback before many accompanying metal-poor stars had a chance to form. In principle, other truncation mechanisms (including reionization) could produce a similar result (see $\S 11$ and 12). 
There are good theoretical reasons to believe that GCs might form in the initial stages of major star forming events. This may be when the gas pressure is highest, favoring the creation of massive compact clusters (e.g., Elmegreen \& Efremov 1997; Ashman \& Zepf 2001). By contrast, the metal-rich GCs are expected to form during a disspational starburst in a more fully-assembled potential well. Here feedback is less effective, and many field stars form. The scenario in which GCs form in the initial stages of a starburst could also qualitatively reproduce a metallicity offset between metal-rich GCs and field stars. Such an offset is observed between the zeropoints of the metallicity-galaxy luminosity relations for metal-rich GCs and field stars (Forbes et al. 1997). However, a difficulty with any such comparison is the existence of radial metallicity gradients in galaxies, since metal-rich GCs show no such gradient. If both metal-poor GC and field star formation are simultaneously truncated at high redshift, their metallicity distributions may show no offset, as is the case in the Galaxy.

GCs in NGC 5128 appear to fall on the structural fundamental plane defined by Galactic and M31 GCs (Harris et al. 2002), although the mean cluster ellipticity in the current (small) sample is higher than that of Galactic GCs and more similar to the distribution in the old GCs of the LMC. Harris et al. also identified six massive GCs that had evidence of extratidal light. This could suggest that these objects are stripped nuclei of dEs, or simply that they are normal GCs, but that King models provide a suboptimal fit to their surface brightness profiles. Widefield photometry in the Washington system by Harris et al. (2004), although complicated by the proximity of NGC 5128 and its low galactic latitude, revealed a total population of $\sim 1000 \mathrm{GCs}\left(S_{N}=1.4\right)$, evenly divided between metalpoor and metal-rich GCs. Peng et al. (2004) presented a large photometric and spectroscopic survey of more than 200 GCs in NGC 5128. 20-25 of the GCs had high enough $\mathrm{S} / \mathrm{N}$ to determine spectroscopic ages and metallicities, and they found a wide range of ages for the metal-rich GCs, with a mean age of $\sim 5$ Gyr. Through direct comparison with integrated spectra of Galactic GCs from Cohen et al.(1998), Peng et al. found some evidence for $[\mathrm{Mg} / \mathrm{Fe}]>0$, although perhaps not as high as in the Galaxy.

Beasley et al. (2005) estimated ages and metallicities for $~ 200$ GCs in NGC 5128 from deep $2 \mathrm{dF}$ spectroscopy (with some overlap with the Peng et al. sample). Using the multi-line fitting method of Proctor et al. (2004), they deduced that the bulk of the GCs in this galaxy can be assigned to one of three old subpopulations: the usual metal-poor and metal-rich subpopulations, plus an intermediatemetallicity subpopulation reminiscent of one discovered in the Virgo gE NGC 4365 (Brodie et al. 2005). Evidence for trimodality is also presented in Woodley, Harris, \& Harris (2005). In Figure 9, histograms of the GC $B-I$ and metallicity distributions show the trimodality in the metallicity distribution. As is readily apparent, the nonlinear color-metallicity relationship combines the metal-poor and intermediate-metallicity peaks into a single peak in the $B-I$ histogram. The presence of old intermediate-metallicity GCs is unusual among massive Es. If these GCs have an accompanying subpopulation of field stars, the field star metallicity distribution could be a combination (albeit not necessarily well-mixed) of intermediate-metallicity and metal-rich stars. However, this would be difficult to detect in present data. In addition to the old subpopulations, about $7 \%$ of the GCs in the Beasley et al. sample appear to be metal-rich with intermediate ages (2-8 Gyr), contrary to previous results. Candidate intermediate-age GCs were specially targeted, so this fraction is probably an upper limit. 
The kinematics of the GCs are similar to those observed in the Galaxy: significant rotation is seen in the metal-rich GCs (as defined in Peng et al. 2004), but only weak rotation is apparent in the metal-poor GCs. This result is contrary to that expected to result from a disk-disk merger. In a merger remnant, the inner parts of the galaxy should display very little rotation because angular momentum is transferred to the outer regions. Of course, the remnant properties will depend on the details of the merger, and a statistical sample of Es that might plausibly have formed in recent major mergers does not yet exist. See $\S 7$ for addition discussion of GC kinematics.

\subsection{Other Galaxies}

An alternative approach to studying the relationship between GCs and field stars has been taken by Forte, Faifer, \& Geisler (2005), who assume that the field star subpopulations follow the radial and color distributions of the GC subpopulations and use galaxy surface photometry to decompose the integrated light into metalpoor and metal-rich constituents. For NGC 1399, they find $T_{b l u e}^{n} \sim 25$ and $T_{\text {red }}^{n} \sim 4$. This metal-rich value is virtually identical to that of the Galaxy and, as before, the metal-poor value is much larger than the metal-rich one. While this approach is potentially a powerful tool for studying field stars in galaxies with unresolved stellar populations, substantial future work will be required to establish the veracity of the key underlying assumption: that the radial and metallicity distributions of GCs and their associated field stars are the same.

\section{Kinematics}

\section{$7.1 \quad$ Ellipticals}

Since GCs are more extended than the stellar light of galaxies, they are useful kinematic tracers of the dark matter halo at large galactocentric radii. GC kinematics also encode detailed signatures of the assembly histories of galaxies; the implications of such observations are just beginning to be understood. Large kinematic samples of GCs ( $>50$ ) have been observed in only four gEs: NGC 1399, M87, NGC 4472, and NGC 5128 (discussed above). Small samples have been studied in several other galaxies, but the numbers are too few to allow the subpopulations to be effectively separated. Aside from noting that there appear to be signatures of strong rotation in the S0s NGC 3115 (Kuntschner et al. 2002) and NGC 524 (Beasley et al. 2004), we will confine our detailed discussion to these four well-studied galaxies.

The largest sample of GC velocities at present exists for NGC 1399, the central $\mathrm{gE}$ in the Fornax cluster. Richtler et al. (2004) have analyzed kinematics for 468 GCs at projected radii of $2-9^{\prime}(\sim 11-49 \mathrm{kpc})$. The velocity dispersions for the metal-poor and metal-rich GCs are $\sim 290 \mathrm{~km} / \mathrm{s}$ and $\sim 255 \mathrm{~km} / \mathrm{s}$, respectively, and do not appear to vary with galactocentric radius. They report a slight tangential bias for the metal-poor GCs, but both subpopulations are generally consistent with isotropic orbits. The larger dispersion for the metal-poor GCs is consistent with their extended spatial distribution. Neither subpopulation shows significant rotation, although a weak signature is observed in metal-poor GCs beyond $6{ }^{\prime}$.

It has been suggested from simulations (Bekki et al. 2003) that NGC 1399 might have stripped the outermost GCs from the nearby E NGC 1404, leaving 
it with an anomalously low $S_{N}$ (NGC 1399 has a high $S_{N}$ of $\sim 5-6$, typical of cluster Es). Bassino et al. (2006) have found that three other Fornax EsNGC 1374, NGC 1379, and NGC 1387-all have $S_{N}$ lower than typical of cluster Es, and also suggest that they may have suffered GC loss to NGC 1399. New GC velocities for NGC 1399, out to $\sim 80 \mathrm{kpc}$, show an asymmetric velocity distribution of the metal-poor GCs at these large radii (Schuberth et al. 2006). This could be interpreted as a signature of GCs that have been stripped from nearby Es but have not yet reached equilibrium in the NGC 1399 potential.

NGC 4472 has been studied by Zepf et al. (2000) and Côté et al. (2003), with $\sim 140$ and $\sim 260$ velocities, respectively, measured over projected radii of $\sim 1.5-8^{\prime}(\sim 7-40 \mathrm{kpc})$. The velocity dispersions of the metal-poor and metalrich GCs are $\sim 340 \mathrm{~km} / \mathrm{s}$ and $\sim 265 \mathrm{~km} / \mathrm{s}$, respectively. The metal-poor GCs rotate around the galaxy's minor axis with a velocity of $\sim 90-100 \mathrm{~km} / \mathrm{s}$; the metal-rich GCs show weak evidence for counter-rotation around the same axis. There is a hint that the outermost (beyond $\sim 25 \mathrm{kpc}$ ) metal-poor GCs may rotate about the major axis, but this conclusion relies on few GCs. The orbits of both subpopulations are consistent with isotropy to the radial limit of the data.

The kinematics of GCs in M87 are rather different from those in either NGC 4472 or NGC 1399. From a sample of $\sim 280$ velocities, Côté et al. (2001) find that both the metal-poor and metal-rich GCs rotate around the minor axis at $\sim 160-170 \mathrm{~km} / \mathrm{s}$, with respective velocity dispersions of $\sim 365$ and $\sim 395 \mathrm{~km} / \mathrm{s}$, when averaged over the whole system. The metal-poor GCs actually appear to rotate around the major axis within $\sim 16 \mathrm{kpc}$ but switch to minor axis rotation only at larger radii. Côté et al. suggest that this change is due to the increasing gravitational dominance of the cluster potential beyond $\sim 18 \mathrm{kpc}$. The GC system as a whole appears quite isotropic, but the data are consistent with a tangential bias for the metal-poor GCs and an opposing radial bias for the metalrich GCs; these biases are poorly constrained in the present sample.

The results for four well-studied gEs (including NGC 5128, discussed in the previous section) present a heterogeneous picture. Although no two galaxies seem to be kinematically similar thus far, we will briefly discuss the extent to which the accumulated information can constrain galaxy formation. K. Bekki and collaborators have used numerical simulations to study the properties of GCs in merging galaxies. Bekki et al. (2002) found that, in disk-disk major mergers, newly formed metal-rich GCs are centrally concentrated and extended along the major axis of the remnant. Angular momentum transferred to the metal-poor GCs causes them to rotate and extend their spatial distribution. These results are strongly dependent on the numerical details of the simulations, and could change if, for example, a different prescription for star formation was used. Simulations of dissipationless major mergers for galaxies with both a disk and bulge extended these results (Bekki et al. 2005). However, the initial conditions (velocity dispersion and anisotropy) of the GC system strongly affect the outcome of such simulations. Pre-existing metal-poor and metal-rich GCs acquire significant amounts of rotation beyond $\sim 10 \mathrm{kpc}$, regardless of the orbital properties of the merging galaxies. Mergers with larger mass ratios leave relatively spherical GC distributions with less rotation. With such a generic prediction of rotation, it is unclear how galaxies like NGC 1399, which shows no rotation in either subpopulation, could have been created. It may be that the merging histories of gEs leave a variety of complex rotational signatures, and that much of the observed differences are due to natural variations in remnant properties and projection 
effects.

It is instructive to consider the results of cosmological simulations of the assembly of dark matter and stellar halos of massive galaxies. Even though the GCs are more extended than the stellar light of the galaxy, they are still more centrally concentrated than the dark matter, so are expected to have a lower velocity dispersion than the dark matter at fixed radii (if the anisotropy of the dark matter and GCs are similar). While providing some constraints, present data sets are still too small to fully determine the anisotropy $\left(\beta=1-v_{\theta}^{2} / v_{r}^{2}\right)$ of the GC system (e.g., Wu \& Tremaine 2006). A wide range of numerical simulations suggest that a general relation holds between $\beta$ and galactocentric radius for both halo tracers and the dark matter: $\beta \sim 0$ (isotropy) in the inner parts, with radial anisotropy increasing outward to $\beta \sim 0.5$ at the half-mass radius of the tracer population (Hansen \& Moore 2004; Dekel et al. 2005; Diemand, Madau, \& Moore 2005; Abadi et al. 2005). Very large samples of GCs $(\sim 500-1000)$ will be needed to test these predictions.

Studies of lower-mass Es are also important, and several groups are pursuing hybrid approaches, using both GCs and planetary nebulae (PNe) to constrain the potential. Romanowsky et al. (2003) suggested, on the basis of PNe kinematics in three "normal" Es (NGC 821, NGC 3379, and NGC 4494), that these galaxies lacked dark matter. A more conventional interpretation is that the observed $\mathrm{PNe}$ originate in stars ejected during mergers and are on highly radial orbits (Dekel et al. 2005). GCs (especially those in the metal-poor subpopulation) are expected to have lower orbital anisotropy than $\mathrm{PNe}$, due both to their extended spatial distribution and the increased probability of destruction for GCs on radial orbits. Two recent studies of GC kinematics in NGC 3379 suggest that the galaxy possesses a "normal" $\Lambda \mathrm{CDM}$ dark halo and are more consistent with the Dekel et al. interpretation (Pierce et al. 2006; Bergond et al. 2006). Thus, GCs may be the best tracers of the mass distribution of Es at large radii.

\subsection{Disk Galaxies}

The best recent review of the kinematics of Galactic GCs is found in Harris (2001). The metal-rich GCs rotate at $\sim 90-150 \mathrm{~km} / \mathrm{s}$, and the rotation rises out to a radius of $\sim 8 \mathrm{kpc}$, beyond which there are few metal-rich GCs. This increasing rotational velocity may represent a transition from bulge to thick disk, though the maximum velocity of $\sim 150 \mathrm{~km} / \mathrm{s}$ is still less than expected for a typical thick disk in a massive spiral. There is little net rotation over the metal-poor subpopulation as a whole. Moreover, no individual radial bin of metal-poor GCs rotates, but somewhat surprisingly, a strong signature of prograde rotation $(140 \mathrm{~km} / \mathrm{s})$ is seen in the most metal-poor GCs $([\mathrm{Fe} / \mathrm{H}]<-1.85)$. This effect is dominated by very metal-poor GCs in the inner halo. The degree of rotational support is similar in this very metal-poor group $(v / \sigma \sim 1.2)$ to the total subpopulation of metal-rich GCs $(\sim 1.3)$.

M31 has been discussed above: the metal-rich GCs rotate with $v / \sigma \sim 1.1$, and the kinematic state of the metal-poor GCs remains unclear. If the identification of the disk objects as young clusters (no matter what the mass) is secure, then the old metal-poor subpopulation is probably pressure-supported.

The situation in M33 is also uncertain, since the ages of many HST-confirmed star clusters are unknown. Chandar et al. (2002) found that the old metal-poor GCs have a velocity dispersion of $\sim 80 \mathrm{~km} / \mathrm{s}$ and are not rotating. They suggested 
that there is a small population of old inner GCs with disk-like kinematics, but an adequate test of this must await a significant increase in the kinematic sample.

Olsen et al. (2004) have suggested that GCs in several Sculptor Group spirals have kinematics consistent with the rotating HI gas disks in these galaxies (in NGC 253, the kinematics are consistent with asymmetric drift from an initial cold rotating disk). Candidates were selected from outside the bright optical disk, so contamination from open clusters in the disk is unlikely to have occurred. Few GCs were observed in each galaxy, and the low systemic velocities of the galaxies inevitably biased the GC selection; those with low velocities cannot be efficiently distinguished from foreground stars. Nonetheless, even with this bias, the lineof-sight velocity dispersions of the GCs are low: from $\sim 35-75 \mathrm{~km} / \mathrm{s}$ out to $\sim 10$ kpc or so. Given the mixed evidence for old disk GCs presented thus far, this preliminary result is worth following up.

The disk galaxies present a somewhat cleaner kinematic picture than the Es, though the number of galaxies studied is still small. If indeed the metal-poor GCs in M31 turn out to be pressure-supported, then this appears to be a common feature of disk galaxies. In the two galaxies with bulges (M31 and the Galaxy) the metal-rich GCs have substantial rotational support. At the very least, these results can serve as valuable starting conditions for simulations of major mergers.

\section{Luminosity Functions}

Excellent reviews of the GC luminosity function (GCLF), including its use as a distance indicator, are given in Harris (2001) and Richtler (2003). The NED D database (2006 release) gives a comprehensive compilation of GCLF distances. Here we summarize some basic facts and discuss the evolution of the GCLF.

In many massive galaxies studied to date, the GCLF can be well-fit by a Gaussian or $t_{5}$ distribution; typical parameters for the normal distribution in Es are peak $M_{V} \sim-7.4$, with $\sigma \sim 1.4$. In spirals, the peak is similar, but $\sigma \sim 1.2$ may be a more accurate value for the dispersion. The peak luminosity is a convolution of the peaks for the two GC subpopulations; line-blanketing effects (especially in bluer bands) cause the metal-poor GC peak to be slightly brighter than the metal-rich peak, so the exact location of the peak depends on the color distribution of the GC system (Ashman, Conti, \& Zepf 1995). The standard peak or turnover of this distribution corresponds to a mass of $\sim 2 \times 10^{5} M_{\odot}$. In absolute luminosity or mass space, this distribution is a broken power law with a bright-end slope of $\sim-1.8$ and a relatively flat faint-end slope of $\sim-0.2$ (McLaughlin 1994). In some massive galaxies there appears to be a departure below a power law for the most massive GCs (e.g., Burkert \& Smith 2000). The expectation of significant dynamical evolution at the faint end of the GCLF has led to suggestions that only the bright half of the GC mass function (GCMF) represents the initial mass spectrum of the GC system. The faint half would then represent the end product of a Hubble time's worth of dynamical destruction. Recent observational and theoretical work on this problem offers some supporting evidence, but also raises some interesting questions.

In the Galaxy, the total sample of GCs is relatively small, especially when divided into subpopulations. This limits our ability to draw general conclusions from this best-studied galaxy. Harris (2001) provided a good overview of the current situation. Within the $8 \mathrm{kpc}$ radius that contains both metal-poor and 
metal-rich GCs, the subpopulations have quite similar LFs. Considering only the metal-poor GCs, the turnover of the GCLF becomes brighter by nearly $0.5 \mathrm{mag}$ out to $8-9 \mathrm{kpc}$, then decreases to its initial value. The inner radial trend is the opposite of naive expectations for dynamical evolution, which should preferentially disrupt low-mass GCs as shocks accelerate mass loss through twobody relaxation. There are too few metal-rich GCs to study the radial behavior of the GCLF in detail. The GCs in the far outer halo (beyond $\sim 50 \mathrm{kpc}$ or so) are all quite faint $\left(M_{V}<-6\right)$, except for the anomalous GC NGC 2419. This cluster has $M_{V}=-9.6$ and may be the stripped nucleus of a dwarf galaxy (van den Bergh \& Mackey 2004). Of course, this level of detail is not expected to be visible in external galaxies, whose GC systems are generally seen in projection about an unknown axis. This is an important limitation in interpreting the results of the theoretical simulations discussed below.

Fall \& Zhang (2001) presented a semi-analytic study of GC system evolution in a Milky Way-like galaxy. The mass-loss rate by two-body relation is taken to depend only on the details of the cluster's orbit, and not on the mass or concentration of the GC. This appears to be consistent with results from more detailed N-body simulations (e.g., Baumgardt \& Makino 2003). Fall \& Zhang found that a wide range of initial GCMFs, including single and broken power laws, eventually evolved to turnover masses similar to those observed. They found that the evolution of the turnover mass was rapid in the first Gyr or two, but that subsequent evolution was slow. The turnover is expected to change substantially with galactocentric radius, due to the preferential destruction of low-mass GCs towards the galaxy center. This can only be avoided if the outer GCs display strong radial anisotropy. However, the assumptions made by Fall \& Zhang need to be considered when applying their results to real data. For instance, they used a static spherical potential, while a live and/or nonradial potential could increase phase mixing and erase some radial signatures of evolution.

Vesperini $(2000 ; 2001)$ modeled the evolution of GCLFs of two initial forms, log-normal and power-law functions. The evolution of individual GCs was determined using analytic formulae derived from the simulations of Vesperini \& Heggie (1997). To summarize their results: GCLFs that are initially log-normal provide a much better fit to the observed data. Dynamical evolution can indeed carve away the low-mass end of a power-law GCLF to produce a log-normal function, but the resulting turnover masses are generally small $\left(\lesssim 10^{5} M_{\odot}\right)$, and vary significantly with galactocentric radius and from galaxy to galaxy. All three predictions for the evolution of an initial power-law function are inconsistent with observations. However, a GCLF that is initially log-normal suffers little evolution in shape or turnover, and that only in the first few Gyr. If constant initial $S_{N}$ is assumed, GC destruction leads to a $S_{N}$-galaxy luminosity relation of the form $S_{N} \propto L^{0.67}$. Vesperini et al. (2003) explored M87 in detail, and (reminiscent of Fall \& Zhang 2001) found that the observed log-normal GCLF, which has a constant turnover with radius (see also Harris et al. 1998), could only evolve from a power-law initial GCLF if there were (unobserved) strong radial anisotropy in the GC kinematics. Of course, the details of the simulations are quite important. For example, Vesperini \& Zepf (2003) showed that a power-law GCLF and a concentration-mass relation for individual GCs can result in a log-normal GCLF with little radial variation in turnover mass. This is because low-concentration GCs are preferentially destroyed at all radii. This is consistent with the study of Smith \& Burkert (2002), who found that the slope of the low-mass part of the 
GCLF in the Galaxy depends upon GC concentration. Another relevant piece of evidence is the similarity in the GCLF turnover among spirals, Es, and even dEs (see \$10.1). This suggests either initial GCLFs close to log-normal (such that little dynamical evolution occurs), or that the dominant destruction processes are not specific to particular galaxy types-for example, disk shocking in spirals.

The sole galaxy with a significant subpopulation of intermediate-age GCs and evidence for dynamical evolution is the merger remnant NGC 1316. HST/ACS observations by Goudfrooij et al. (2004) have clearly demonstrated dynamical evolution of its GC system. When they divided the red $(1.03 \leq V-I \leq 1.40)$ GCs into two equal radial bins, they found that the outer (beyond $\sim 9.4 \mathrm{kpc}$ ) GCs have the power law LF seen for many systems of YMCs in merging and starbursting galaxies. By contrast, the inner GCs show a LF turnover characteristic of old GC systems. As Goudfrooij et al. argued, these observations would appear to provide the conclusive link between YMCs in mergers and old GCs in present-day Es.

However, the difficulty with this interpretation is that the observed location of the metal-rich peak of the inner GCs in NGC 1316 is at $M_{V} \sim-6$. If the metalrich GCs were formed in the merger, are $\sim 3$ Gyr old, and have solar metallicity (consistent with spectroscopic results; Goudfrooij et al. 2001), Maraston (2005) models with either a Salpeter or Kroupa IMF predict that age-fading to $12 \mathrm{Gyr}$ will result in a peak at $M_{V} \sim-4.6$. Old metal-rich GCs are observed to have a GCLF turnover at $M_{V} \sim-7.2$ (e.g., Larsen et al. 2001). Thus $\sim 2.6 \mathrm{mag}$ of additional evolution of the GCLF turnover $(\sim 1.2 \mathrm{mag}$ of dynamical evolution and $\sim 1.4$ mag of age fading) would be required to turn the new metal-rich GC subpopulation of NGC 1316 into that of a normal E. The $1.2 \mathrm{mag}$ of dynamical evolution needed is far beyond that predicted by even the most "optimistic" models for GC destruction. For example, Fall \& Zhang (2001) models for GC system evolution, assuming a power-law initial GCMF, predict $<0.2$ mag of evolution in the GCLF peak from 3 to 12 Gyr using the same Maraston (2005) models. This is consistent with the finding of Whitmore et al. (2002) that little evolution in the GCLF peak is expected after the first 1.5-2 Gyr, as age-fading balances GC destruction. The implication is that in a Hubble time, the metalrich GC system of NGC 1316 may not look like that of a normal E galaxy. Fall \& Zhang (2001) models, designed to study GC destruction in a Milky Waylike galaxy, may even have limited applicability to NGC 1316. Other models (e.g., those of Vesperini discussed above) predict less evolution, so the expected difference between the suitably evolved young GCs in NGC 1316 and the old metal-rich GCs in local Es could be even greater. A caveat here is that the observed GCLF peak could be the convolution of a brighter, more evolved GC population in the innermost regions with a less evolved population in the outer parts of the bin. Such a convolution would tend to lessen the difference between the observed and expected turnover luminosity.

Thus, while there is some evidence for the paradigm of the evolution of a powerlaw LF to a log-normal LF, there are still unresolved issues. The installation of WFC3 on a potential future HST servicing mission would allow much more efficient and accurate age-dating of YMCs through its wide-field $U$-band imaging capability. This would also make it possible to investigate the mass function as a function of age in more detail, and hence directly address questions of dynamical evolution. 


\section{Sizes}

The present-day appearance of a GC is a complicated convolution of the initial conditions of its formation with subsequent internal and external dynamical effects. The surface brightness distributions of GCs are reasonably well-fit by single-mass King (1966) models. These form a dimensionless one-parameter family as a function of the concentration $c=r_{t} / r_{0}$ (where $r_{t}$ is the tidal radius and $r_{0}$ is the scale radius). In dimensionless form, the half-light radius ( $r_{h}-$ "size") is a monotonic function of $c$, and is the only one of these three radii that is relatively unaffected by dynamical evolution (Spitzer 1987; Meylan \& Heggie 1997) and can serve as a probe of GC formation conditions.

A correlation between $r_{h}$ and galactocentric radius $(R)$ for Galactic GCs was discovered by van den Bergh et al. (1991). This could not be explained by dynamical evolution of the GC system, since diffuse inner GCs might be expected to be destroyed, but compact distant GCs should (if they existed) have remained intact. Thus, this result represents strong evidence for some degree of in situ formation of Galactic GCs.

HST/WFPC2 imaging of NGC 3115 and M87 revealed that metal-poor GCs are $\sim 20 \%$ larger than metal-rich GCs (Kundu \& Whitmore 1998; Kundu et al. 1999). This was confirmed statistically in many early-type galaxies by Larsen et al. (2001) and Kundu \& Whitmore (2001a). Explanations offered for this result have included:

(i) It represents an intrinsic formation difference, e.g., the metal-rich GCs formed in a higher-pressure environment.

(ii) It is a results of projection effects. Since the metal-rich GC spatial distribution is more centrally concentrated than that of the metal-poor GCs, within some given projected radius the metal-rich GCs will tend to lie at smaller $R$. If there is a strong correlation between size and $R$ (as in the Galaxy), the metal-rich GCs will appear smaller on average than the metal-poor GCs (Larsen \& Brodie 2003). This model predicts that size differences will be largest in the inner parts of galaxies and disappear in the outer regions.

(iii) It is a natural outcome of assuming metal-poor and metal-rich GCs have the same half-mass radii. Since the brightest stars in metal-rich GCs are more massive than in metal-poor GCs, mass segregation leads to a more compact distribution and a smaller half-light radius (Jordán 2004). In this model there should be little change in the relative sizes with galactocentric distance.

Option (i) is not testable at present, so should be left as a fallback only if the other possibilities can be eliminated. Regarding (ii), Larsen \& Brodie (2003) showed that the $r_{h}-R$ correlation in the Galaxy could explain all of the observed size differences between the metal-poor and metal-rich GCs. However, in order to explain the $\sim 20 \%$ size difference in external galaxies, steep $r_{h}-R$ relations would be required, and the radial distribution of the GCs would need to have a central core like a King profile (this appears to be consistent with observations). Model (iii) has a number of critical assumptions upon which its conclusions depend, including identical GC ages and initial mass functions. Small changes in either of these parameters (e.g., an age difference of $\sim 2$ Gyr between the metal-poor and metal-rich GCs) could erase most of the expected size difference. Jordán (2004) also used equilibrium King-Michie models to represent the GCs; full Nbody modeling is a desirable next step. In Figure 10, taken from Jordán (2004), we show the sizes of GCs in M87 together with a best-fit model of type (iii). 
Several recent observational results have provided important new constraints. Jordán et al. (2005) studied GC sizes in 67 early-type galaxies with a wide range of luminosity from the ACS Virgo Cluster Survey (Côté et al. 2004). For bright metal-poor GCs they found a significant but rather shallow relationship between $r_{h}$ and projected $R$ (normalized to the effective radius of the galaxy). In log space the value of the slope is 0.07 , compared to $\sim 0.30$ for a similar sample of Galactic GCs. They did not list the fits for individual galaxies, and there are clearly variations, but the bulk of the galaxies do not appear to have $r_{h}-R$ relations as steep as observed in the Galaxy, so projection effects on the GC subpopulation sizes should be small, and option (ii) is not favored. They also found that GCs in bluer/fainter host galaxies tend to be slightly larger. Despite these variations, $r_{h}$ is still relatively constant among galaxies. Thus, with their observed correlations between $r_{h}$ and galaxy properties, they were able to calibrate $r_{h}$ as a distance indicator, as suggested by Kundu \& Whitmore (2001a).

Wide-field HST/ACS data for NGC 4594 (covering $\sim 6^{\prime} \times 10^{\prime}$ ) do not appear consistent with option (iii), however. Spitler et al. (2006) found that the ratio of metal-poor to metal-rich GC sizes declines steeply and steadily from $\sim 1.25$ in the center to $\sim 1$ at the edge of the complete observations. Thus it appears that, at least in NGC 4594, projection effects account for most of the observed size differences.

It seems clear from these results that both projection and segregation mechanisms can play a role in determining the sizes observed for extragalactic GCs. Since each is sensitive to the physical conditions of the GC system, galaxies will need to be studied on an individual basis to determine which effects are important. Particularly valuable would be high-resolution, wide-field imaging of GC systems, like the HST/ACS mosaic of NGC 4594 discussed above.

\section{Dwarf Galaxies}

It has been known for a long time that many dwarf galaxies have GC systems (e.g., Fornax dSph; Shapley 1939). In the Galaxy formation model of Searle \& Zinn (1978) and in many subsequent studies of GC formation, it was envisioned that metal-poor GCs form in protogalactic dwarf-sized clumps (e.g., Harris \& Pudritz 1994; Forbes et al. 1997; Côté et al. 1998; Beasley et al. 2002). The dwarf satellites around massive galaxies like the Milky Way can then be interpreted as the remnants of a large initial population of such objects, most of which merged into the forming protogalaxy. If this process happened at high redshift, most of the fragments could still have been be gaseous and thus have formed stars and/or contributed gas as they merged. At lower redshift the process could be primarily dissipationless, as envisioned by Côté et al. and seen in action through the present-day accretion of the Sgr dSph. What can GCs tell us about this process?

The faint end of the galaxy luminosity function is uniquely accessible in the Local Group. Forbes et al. (2000) provided a good census of GCs in Local Group dwarfs, which has changed only marginally since that time. The candidate GC in the dIrr Aquarius is apparently a yellow supergiant (D. Forbes, private communication), so the lowest-luminosity Local Group galaxies with confirmed GCs are the Fornax $\left(M_{V}=-13.1\right)$ and $\operatorname{Sgr}\left(M_{V}=-13.9\right)$ dSphs, each of which has at least five GCs. The recently discovered CMaj dSph (Martin et al. 2004) ap- 
pears to have at least four GCs whose properties are distinct from the bulk of the Galactic GC system (Forbes, Strader, \& Brodie 2004). At least two of the GCs in each of Sgr and CMaj are of intermediate age and metallicity. The LMC has a subpopulation of old metal-poor GCs and a famous "age gap" between the old GCs and a subpopulation of intermediate-age GCs ( $\sim 3$ Gyr). It also hosts a number of younger clusters, some of which, like GCs, might be massive enough to survive a Hubble time (e.g., Searle, Wilkinson, \& Bagnuolo 1980; van den Bergh 1994). The SMC has only one old GC but a more continuous distribution of massive clusters to younger ages (Mighell et al. 1998). Together these results suggest star formation histories that were at least moderately bursty (e.g., Layden \& Sarajedini 2000). Lower-mass Galactic dwarfs (e.g., Leo I; $M_{V} \sim-12$ ) do not have GCs. Less is known about the GC systems of similar-mass M31 dwarfs. Grebel et al. (2000) suggest a candidate GC in And I ( $\left.M_{V} \sim-12\right)$, but this GC could be a contaminant from M31. Outside the Local Group, Sharina et al. (2003) spectroscopically confirmed a GC in the M81 dSph DDO 78, which has a mass intermediate between Fornax and And I. Karachentsev et al. (2000) identified candidate GCs in a number of other M81 dwarfs, but these have not yet been confirmed.

As discussed in Strader et al. (2005), these observations put important constraints on the minimum mass of halos within which metal-poor GCs could form. Fornax and Sgr have total masses of $\gtrsim 10^{8} M_{\odot}$ (Walcher et al. 2003; Law et al. 2005) and the total mass estimate for And I, assuming it has a similar M/L ratio, is a few $\times 10^{7} M_{\odot}$. This suggests that, at least in a relatively low-density group environment, GCs formed in halos with minimum masses of $\sim 10^{7}-10^{8} M_{\odot}$. Whether GCs typically form in groups of GCs or alone is unknown. Fornax and Sgr each have several GCs, and the dIrr NGC $6822\left(M_{V} \sim-15.2\right)$ has up to three old GCs, though two of these are located far from the main body of the galaxy (Cohen \& Blakeslee 1998; Hwang et al. 2005). The dIrr WLM $\left(M_{V} \sim-14.5\right)$ has only one old GC, which is metal-poor (Hodge et al. 1999). A caveat to these arguments is that in some models (e.g., Kravtsov, Gnedin \& Klypin 2004), present-day dwarf satellites have undergone significant stripping of dark matter, and may have been much more massive initially $\left(\gtrsim 10^{9}-10^{10} M_{\odot}\right)$. However, detailed comparisons between observed velocity dispersion profiles and numerical simulations suggest little mass loss due to tidal stripping for well-studied Galactic dSphs (Read et al. 2006). It is important to realize that differences in baryonic mass loss (e.g., due to stellar feedback; Dekel \& Silk 1986) may modify the amount of stellar mass in galaxies of similar halo mass. GC kinematics (see §10.3) offer one of the best routes to directly determine the total masses of dwarfs outside the Local Group.

\subsection{Specific Frequencies and Luminosity Functions}

GC systems were discovered around 11 Virgo dwarf ellipticals $\left(\mathrm{dEs}^{2}\right)$ using groundbased (CFHT) imaging (Durrell et al. 1996). The $S_{N}$ of these galaxies is relatively

\footnotetext{
${ }^{2}$ In deference to common usage, here we utilize the term "dwarf elliptical" for early-type galaxies with low luminosities $\left(M_{B} \lesssim-18\right)$. The structural parameters of many of these galaxies differ from those of "classical" Es (e.g., Kormendy 1985; Kormendy 1987) and may well suggest a different formation history. They are sometimes called spheroidal ( $\mathrm{Sph}$ ) galaxies. A small number of galaxies in this luminosity range have structural parameters consistent with powerlaw Es (see $\S 4)$.
} 
high, $\sim 3-8$, and the GC systems are very centrally concentrated: most GCs are within $<30^{\prime \prime}(2 \mathrm{kpc})$ at the distance of Virgo, while a typical $\mathrm{dE}$ has a half-light radius $\lesssim 1 \mathrm{kpc}$. Strader et al. (2006) obtained radial distributions that were consistent with these earlier results, except for a few bright dEs $\left(M_{V} \lesssim-18\right)$ where the outermost GCs were found at 7-9 kpc. This is near the limit of the radial coverage of $H S T$ /ACS at the distance of Virgo, so it is possible that GCs may be found at even larger radii.

Miller et al. (1998) used HST/WFPC2 snapshot imaging to explore the specific frequencies of a large sample of dEs in the Virgo and Fornax clusters, including galaxies with luminosities as faint as $M_{B} \sim-13$. They found a dichotomy between nucleated $(\mathrm{dE}, \mathrm{N})$ and non-nucleated $(\mathrm{dE}, \mathrm{noN})$ galaxies. $\mathrm{dE}, \mathrm{noN}$ dwarfs appeared to have low $S_{N}$ values $(\sim 3)$, independent of galaxy luminosity. dE,N galaxies had higher $S_{N}$ and showed an inverse correlation between $S_{N}$ and luminosity. It has been suggested that $\mathrm{dE}$ galaxies may have originated as dIrrs or low-mass disk galaxies (e.g., Moore, Lake, \& Katz 1998; see discussion below). Miller et al. argued that few $\mathrm{dE}$ galaxies could have formed in this manner, as the $S_{N}$ values even for $\mathrm{dE}, \mathrm{noN}$ galaxies are larger than expected from age-fading such hosts.

Strader et al. (2006) revisited these findings in an HST/ACS study of Virgo Es which included $37 \mathrm{dEs}$. 32 of these have structural parameters consistent with "true" dEs; the other five appear to be faint power-law Es (Kormendy et al. 2006 and private communication). The ACS images offered superior areal coverage and depth compared to those available to Miller et al., but the Strader et al. sample spanned a smaller luminosity range: $-15 \lesssim M_{B} \lesssim-18$. It was not possible to investigate the differences between $\mathrm{dE}, \mathrm{N}$ and $\mathrm{dE}, \mathrm{noN}$ galaxies, since many of the galaxies previously classified as dE,noNs either have faint nuclei or are power-law Es. There may be few true dE,noNs with $M_{B} \lesssim-15$ in Virgo. Strader et al. found no strong correlation between galaxy luminosity and $S_{N}$ for either dEs or faint power-law Es. The faintest galaxies might have larger $S_{N}$, but the effect is not strong. The lack of a $S_{N}-L$ trend could be due to the more restricted luminosity range of galaxies studied by Strader et al.compared to Miller et al..

Interestingly, a bimodal distribution of $S_{N}$ values was discernible in their sample. As shown in Figure 11, more than half of the galaxies were found to have $S_{N} \sim 1$, while the $S_{N}$ values of the remainder ranged from 3 to 10, with a median at $\sim 5$. This difference spans the observed luminosity range and does not correlate with either the presence of a nucleus or the color distribution of the GCs. A natural interpretation of the $S_{N}$ differences is that they reflect multiple formation channels for dEs in Virgo. Mechanisms for forming dEs include "harassment", the cumulative effect of many high-speed galaxy encounters (Moore et al. 1996), stripping or age-fading of low-mass disks (Kormendy 1985), or processes similar to those responsible for the formation of more massive Es (this many be most applicable to the faint power-law Es). It is possible that the high $S_{N}$ galaxies (the Fornax dSph with $S_{N} \sim 29$ is an extreme example) simply represent those in which stellar feedback during the first major starburst was very effective (e.g., Dekel \& Silk 1986). However, if this is the case, a signature should be apparent in the GC color distributions. In particular, the presence of metal-rich GCs (see below) and a continuous (rather than bimodal) distribution of $S_{N}$ might be expected. Certainly, feedback will be increasingly important with decreasing galaxy mass, and would provide a simple explanation for a relation between $S_{N}$ 
and luminosity, should one be confirmed. Photometric, structural, and kinematic studies of these same dEs will be needed to discriminate among the many possible explanations.

These same studies have also afforded the ability to study the GCLF in dEs. The power law slope $(\sim-1.8)$ measured by Durrell et al. (1996) for the massive end of the GCLF in their Virgo dEs is the same as that found in normal Es. However, they measured a GCLF turnover $\left(M_{V} \sim-7.0\right)$ that is fainter by $\sim 0.4-0.5 \mathrm{mag}$ than that typical for massive galaxies. This result may have been influenced by the difficulty of rejecting contaminants in ground-based data. Strader et al. (2006) constructed a composite of the $37 \mathrm{dEs}$ in their HST sample, using the outer parts of the images to correct for background contamination. In contrast to Durrell et al., they found that the dE GCLF peak occurs at the same value as in the massive gEs in their sample (M87, NGC 4472, NGC 4649). This comparison was made in $z$, where there is little dependence of cluster $M / L$ on metallicity over the relevant range, so the differences in GC color distributions between gEs and dEs should not affect the GCLF comparisons.

That the GCLF peaks for the dwarfs and the giants match so well in the Strader et al. study is perhaps puzzling. The theoretical expectation is that in low-mass galaxies dynamical friction will act to deplete the GC system, preferentially destroying massive GCs. Such GCs will spiral into the center in less than a Hubble time, forming or contributing to a nucleus. Lotz et al. (2001) performed a semi-analytic study of this phenomenon, and found that dynamical friction is expected to produce more luminous nuclei than observed. Strader et al. (2006) found that the luminosities of a subset of dE nuclei are consistent with formation through dynamical friction, but that the majority appear to be formed by a separate mechanism. This point, together with the similarity of the GCLF turnovers, implies that dynamical friction has not had a substantial effect on the GC systems of dEs. Lotz et al. (2001) suggested several explanations for the lack of observable consequences of dynamical friction, including extended dark matter halos around dEs, or tidal torquing of GCs (this latter explanation was also proposed for the Fornax dSph by Oh et al. 2000). Goerdt et al. (2006) have used numerical simulations to show that the dynamical friction timescale in Fornax is longer than a Hubble time if its dark matter halo has a core (instead of the cusp generically predicted in galaxy formation in $\Lambda \mathrm{CDM})$. Kinematic studies of GCs in dEs, such as those of Beasley et al. (2005), are also beginning to build a better understanding of the halo potentials of dwarf galaxies.

So far we have included little discussion of dIrrs. Their GC systems are quite difficult to study. Indeed, the contrast in our understanding of dEs and dIrrs is analogous to the information gap between Es and spirals. The relatively small GC systems of dIrrs, their ongoing star formation, and the resulting inhomogeneity of the background are serious observational challenges. A HST/WFPC2 study of 11 Virgo and Fornax dIrrs by Seth et al. (2004) found typical $S_{N}$ values of $\sim 2$, but uncovered two galaxies with much higher $S_{N}$. Stellar M/L values are low in typical dIrrs, which suggests that the $S_{N}$ values will become much higher after nominal age-fading of the dIrr, as might be expected in transformation to a dE. However, many of the detected objects are unlikely to be old GCs. In the Local Group, the Magellanic Clouds and NGC 6822 each have a population of massive intermediate-age GCs (in addition to a small number of old GCs) that reflect the extended star formation histories of these galaxies. The same may be true of cluster dIrrs. Spectroscopy will probably be needed to determine 
the present fraction of intermediate-age GCs, which is crucial for isolating the differences in the formation histories of the various classes of dwarf galaxies. The combination of optical and NIR photometry, once the SSP models have been sufficiently calibrated for old GCs, offers a promising future tool for identifying younger clusters.

\subsection{Color Distributions}

The classical view of dwarf galaxy GCs is that they are uniformly metal-poor. This is supported by a "combined" metallicity distribution of old GCs in Local Group dwarfs (Minniti et al. 1996), which peaks at $[\mathrm{Fe} / \mathrm{H}] \sim-1.8,0.3$ dex more metal-poor than that of the Galactic metal-poor GC subpopulation.

We now know that reality is more complicated. As noted above, the Sgr dSph (and perhaps the CMa dSph) have two GCs of intermediate metallicity and age. An HST/WFPC2 study of the color distributions of dEs in Virgo and Fornax (Lotz et al. 2004) revealed a rather wide spread in color, consistent with the presence of metal-rich GCs. However, it was not possible to distinguish subpopulations in their small GC samples.

Sharina et al. (2005) have published a study of GCs in a large sample of dSphs and dIrrs using a heterogeneous set of HST/WFPC2 images. The metal-poor GC peak appears to be $\sim 0.1$ redder in $V-I$ than the peak found by Lotz, Miller, \& Ferguson (2004) for their sample of dEs. There is no ready explanation for this finding, which is inconsistent with previous work. It may be that a photometric zero-point offset is to blame, as suggested by Sharina et al. themselves. The GC color distributions of both galaxy types have a tail to redder values. This may reflect a small subpopulation of metal-rich GCs, or could be due to contamination by background objects. There may also be a few metal-rich GCs in the dIrrs studied by Seth et al. (2004).

The existence of metal-rich GCs in dEs has been shown conclusively by Peng et al. (2006) and Strader et al. (2006). A large fraction of Virgo dEs, down to quite faint magnitudes $\left(M_{B} \sim-15\right)$, were found to have bimodal color distributions, analogous to those observed in massive Es. The slope of the metal-rich GC colorgalaxy luminosity relation is not well-constrained at these low luminosities due to the small number of GCs associated with each galaxy. The new data points are consistent with either: (i) a linear extrapolation to lower magnitudes and bluer colors from the region of massive galaxies, or (ii) a slight flattening at the low mass end of the relation. It is reasonable then to ask whether these metal-rich GCs (or at least a subset) could have intermediate ages, like the two younger GCs discovered in the Sgr dSph. Beasley et al. (2005) obtained high-quality spectra for three metal-rich GCs in the Virgo dE VCC 1087. Their old ages are consistent with those of the metal-poor GCs within the errors. Although spectra of similar quality for a large sample of dEs clearly would be desirable, the results to date suggest that there is no obvious dichotomy in the color distributions of $\mathrm{dEs}$ and massive Es.

Using data from Peng et al. (2006), Forbes (2005) has pointed out a possible link between GC bimodality in dEs and the galaxy color bimodality observed in large surveys (e.g., Bell et al. 2004) below a critical mass of $\sim 3 \times 10^{10} M_{\odot}$. Above this mass, nearly all galaxies in the Virgo Cluster Survey have bimodal GC systems; below this mass, an increasing fraction of galaxies have unimodal color distributions. One interpretation of this phenomenon is that the critical mass 
represents a transition from "cold", smooth accretion of gas into halos below the critical mass to "hot" accretion of gas that shocks at the virial radius and is unable to form stars (e.g., Dekel \& Birnboim 2006).

\subsection{Kinematics}

Kinematic studies of GCs in dwarfs are challenging, principally because of the small GC systems and lack of luminous GCs. Puzia et al. (2000) found that the velocity dispersion of seven GCs in the luminous dE NGC 3115 DW1 suggested a relatively high $M / L_{V} \sim 22 \pm 13$. This could suggest the presence of dark matter, or that its parent S0 NGC 3115 is stripping the outermost GCs.

In the Virgo dE VCC 1087, the GCs rotate at $\sim 100 \mathrm{~km} / \mathrm{s}$ around the minor axis (Beasleyet al. 2005). The sample of twelve GCs is dominated by metal-poor GCs, although it includes three GCs whose colors and spectroscopic metallicities are consistent with a metal-rich subpopulation (such subpopulations appear to be common in Virgo dEs; see $\S 2$ and 10.2). Its GC system has the largest rotational support of any galaxy studied to date, with $v / \sigma \sim 3.6$, typical of a disk. This makes VCC 1087 a prime candidate for a dE that evolved from a disky dIrr. We note in passing that, although the LMC is often considered to have a rotating disk population of old metal-poor GCs (e.g., Schommer et al. 1992), van den Bergh (2004) has argued that the current data do not strongly discriminate between disk and halo kinematics for these GCs.

\section{Globular Cluster Formation}

\subsection{Classical Scenarios}

In $\S 2.1$ we described the three principal scenarios that have been suggested as explanations for GC bimodality: major disk-disk mergers, in situ formation through multiphase dissipational collapse, and dissipationless accretion. How do these models account for the other observed properties of GC systems? Here we discuss the arguments made in the literature for and against these scenarios, as well as additional constraints from newer data described in this article.

11.1.1 MAJor Mergers As noted in $\S 2.1$, the observation of young massive star clusters in many merger remnants throughout the 1990s gave a significant boost to the major disk-disk merger model for GC bimodality (Ashman \& Zepf 1992). While some of these objects definitely have masses and sizes that should allow them to evolve into old GCs (e.g., Maraston et al. 2004; Larsen, Brodie, \& Hunter 2004), others may have abnormal IMFs that preclude their long-term survival (e.g., McCrady, Gilbert, \& Graham 2003; Smith \& Gallagher 2001; Brodie et al. 1998), though important uncertainties in dynamical mass estimates due to mass segregation remain (McCrady, Graham, \& Vacca 2005). In a broader context, despite the fact that YMCs and GCs are remarkably similar in many respects, it remains unclear whether (after a Hubble time of evolution) young GC systems will have properties consistent with those of old GC systems in local galaxies. The issue here is that observations of intermediate-age GCs may be at odds with the expected signatures of a simple dynamical evolution scenario (see $\S 8)$.

Even before color bimodality had been observed, several authors used GCs to constrain the feasibility of the disk-disk merger picture for forming Es. Harris 
(1981) and van den Bergh (1984) noted that typical Es had more populous GC systems than spirals. Massive disk galaxies have $S_{N} \sim 1$; Es have $S_{N} \sim 2-5$ depending on environment, with even higher values for brightest cluster galaxies (BCGs) like M87. This is often termed the " $S_{N}$ problem". Schweizer (1987) explicitly addressed this concern by suggesting that many new GCs might be formed in the merger. As has been pointed out by multiple authors, this will only raise the $S_{N}$ if GCs form with a higher efficiency relative to field stars than they did in the protogalactic era. Since GC formation efficiency appears to increase with star formation rate, $S_{N}$ may only increase if the star formation rate in a present-day merger is higher than it was when the GCs in spirals were originally formed.

Several other problems with the major merger model were pointed out in Forbes et al. (1997). For example, they showed that there is a correlation between $S_{N}$ and the fraction of metal-poor GCs, such that the highest $S_{N}$ galaxies also have the highest proportion of metal-poor GCs. However, the major merger scenario predicts the opposite behavior: the mechanism to increase the $S_{N}$ of spirals is the formation of new metal-rich GCs in the merger; this should result in larger metalrich GC subpopulations in more massive Es. Ashman \& Zepf (1998) gave a candid analysis of the then current situation on the merger front and suggested that the gEs that dominated the high $S_{N}$ end of the Forbes et al. relation could be expected to have augmented their metal-poor GC population by the accretion of lower-mass galaxies (see below) during their complex formation histories. Moreover, they pointed out that the $S_{N}$ values in the literature were likely to be very uncertain because so few galaxies had been scrutinized with high-quality wide-field imaging. As discussed in $\S 3$, more recent work confirms that $S_{N}$ values for Es tend to come down with improved observations. In general, however, there are still fewer metal-rich than metal-poor GCs in present-day Es, and this remains in conflict with the major merger prediction. Rhode et al. (2005; see §3.1) considered the $S_{N}$ (or $T$ ) values of the individual subpopulations and concluded that massive cluster Es cannot have been formed from mergers of local spirals, although some lower-mass field Es could still have formed in this manner. Harris (2001) reached essentially the same conclusion from an analysis of the required gas content and GC formation efficiencies.

Another constraint on the major merger model can be found in the metalpoor GC metallicity-galaxy luminosity correlation (Strader et al. 2004a). In the mean, the metal-poor GC subpopulations of spirals have lower metallicities than those of massive Es. This seems to be a strong argument against the major merger scenario. However, this conclusion does not take into account the expected effects of biasing - see $\$ 11.2$. It is notable that even some low-mass dEs have bimodal GC color distributions that follow the same peak relations as massive galaxies $(\S 10.2)$, even though these galaxies have presumably not suffered a major merger. So, even if major disk-disk mergers were a viable route to producing bimodality in some cases, they could not be the sole process in operation. In addition, the ages of metal-rich GCs in Es (see §4) imply a formation epoch $z \gtrsim 2$. This restricts most putative major mergers to higher redshifts.

As discussed in $\S 2.1$, the Forbes et al. (1997) multi-phase collapse scenario arose as a response to issues with the merger model. There has been little observational evidence to date against the Forbes et al. scenario, but, to a considerable extent, this is because it made few specific predictions of observable quantities. Its usefulness was as a framework within which to consider alternative explanations for 
GC color bimodality, and it identified aspects of the picture still under consideration, e.g., the need to truncate GC metal-poor formation at high redshift. More recent scenarios described below in $\S 11.2$ are generally consistent with this broad framework.

We emphasize that the arguments presented here against the major merger scenario apply principally to the formation of massive Es from present-day spirals with relatively small bulges. Current GC observations are consistent with dissipational formation of Es at relatively high redshift $(z \gtrsim 2)$-including major mergers, as long as the disk progenitors have higher $S_{N}$ than spirals in the local universe. Subsequent dissipationless merging could then form the most massive gEs, under the constraint of "biased" merging discussed in $\S 11.2$.

11.1.2 Dissipationless ACCREtion The accretion scenario of Côté et al. (1998) was explicitly designed to be consistent with hierarchical structure formation. It assumes a protogalactic GC metallicity-galaxy mass relation produced through a dissipational process at high redshift. The GC systems of present-day galaxies are envisaged to have formed through subsequent dissipationless merging. Since in this scenario the intrinsic GC metallicities of massive protogalaxies are quite high, such galaxies must accrete large numbers of metal-poor GCs from dwarf galaxies to produce bimodality. Côté et al. (2002) used Monte Carlo simulations to show that the acquisition of the necessary numbers of low-metallicity GCs required the low-mass end of the the protogalactic mass function to have a very steep slope $(\sim-2)$. However, even with such a steep mass function, Ashman, Walker, \& Zepf (2006) found that, when they ran simulations similar to those of Côté et al., color distributions like those observed in massive galaxies occurred in only a small fraction $(\sim 5 \%)$ of their simulations. Another potential problem is that the accreted dwarfs would be expected to contribute many metal-poor field stars that are not observed, unless the dwarfs are primarily gaseous (e.g., Hilker 1998).

The fact that metal-poor GCs in dwarfs have much lower metallicities than those in massive Es (by 0.5-0.6 dex) would, at first sight, seem to be direct

evidence against the accretion scenario. This argument was made in Strader et al. (2004a). However, this line of reasoning does not account for the effects of biased structure formation, which may be the key to properly understanding the implications of the metal-poor GC metallicity-galaxy mass relation.

As already emphasized, in the light of our current understanding of hierarchical galaxy assembly, all galaxy formation scenarios must be accretion/merger scenarios at some level. The major merger and the accretion models (as published) both provided an important focus for theoretical discussion and observational effort by making fairly explicit predictions against which the observations could be compared. The preponderance of new evidence now suggests that, while elements of each remain viable, the details are pointing us in new directions (see below).

\subsection{Hierarchical Merging and Biasing: Recent Scenarios}

Beasley et al. (2002) explored GC bimodality in a cosmological context using the semi-analytic galaxy formation model of Cole et al. (2000), and this work contained elements of all three classic scenarios. While largely phenomenological, it makes the most specific predictions of any model proposed, and because the scenario is in the context of a full model of cosmological structure formation, it implicitly accounts for many of the issues discussed below (e.g., biasing). Metal- 
poor GCs were assumed to form in the early universe in gas disks in low-mass dark matter halos. As in Forbes et al. (1997), Beasley et al. found it necessary to invoke the truncation of metal-poor GC formation at high redshift (in this case, $z>5$ ) in order to produce bimodality. The metal-rich GCs were generally formed during gas-rich mergers. Their predictions for the metal-rich subpopulation included: a correlation between GC metallicity and galaxy luminosity, significant age and metallicity substructure, and decreasing mean ages and metallicities in lowerdensity environments.

Following suggestions from Santos (2003), Strader et al. (2005) and Rhode et al. (2005) proposed hierarchical scenarios for GC formation intended to account for the metal-poor GC metallicity-galaxy mass relation and the correlation of metal-poor $S_{N}$ (or $T_{\text {blue }}$ ) with galaxy mass. Metal-poor GCs are proposed to form in low-mass dark matter halos at very high redshift, typically $z \sim 10-15$. Halos in high-density environments collapse first. As discussed in Strader et al., this scenario can reproduce the observed correlations with galaxy mass, given reasonable assumptions (including the truncation of metal-poor GC formation at high $z$, plausibly by reionization). It can also explain other observations, such as the radial distribution of metal-poor GCs (Moore et al. 2006) and possibly the mass-metallicity relation for individual metal-poor GCs (Strader et al. 2006; Harris et al. 2006). Metal-rich GCs form in the subsequent dissipational merging that forms the host galaxy. When the bulk of this "action" took place is not wellconstrained but, for galaxies at $\sim L^{*}$ and above, most GCs appear to have formed at $z \gtrsim 2$ (Strader et al. 2005; Puzia et al. 2005). Some additional dissipationless merging for massive Es appears to be required, based on the evolution of the "red sequence" luminosity function of early-type galaxies from $z \sim 1$ to the present (Faber et al. 2005; Bell et al. 2004) and the dichotomy of core parameters (§4.1). The ages of metal-rich GCs do not constrain such dissipationless merging, but in the future the radial distributions and kinematics may offer interesting insights. We call this picture of GC formation the synthesis scenario.

It has been mentioned several times in the preceding sections that biasing is a key factor in understanding structure formation. Could the metal-poor GC subpopulations of massive Es have been built from major mergers of present-day lower-mass disk galaxies or by the accretion of many dwarf galaxies, since both dwarfs and spirals have metal-poor GCs of lower metallicity than those in massive Es?

To simultaneously accommodate: (i) the metal-poor GC metallicity-galaxy mass relation and (ii) the theoretical and observational evidence that most massive galaxies have undergone some degree of merging/accretion since $z \sim 2$, we must argue that the metal-poor GC relation was different at higher redshift. A present day $L^{*}$ galaxy cannot have been assembled from present-day sub- $L^{*}$ galaxies. Instead, the merging must have been biased, in the sense that galaxies with metal-poor GC systems that would lie above the relation connecting GC metallicity and host galaxy mass at $z=0$ would tend to have merged into more massive galaxies by the present (see Figure 12 for a schematic diagram of this process). This can be understood as a direct result of hierarchical structure formation: high- $\sigma$ peaks in the most overdense regions (destined to become, e.g., galaxy clusters) collapse and form metal-poor GCs first. These metal-poor GCs will be more highly enriched than those forming in halos that collapse later, either because they have more time to self-enrich, or because the density of nearby star-forming halos is larger and they could capture more outflowing enriched gas. 
These first-forming metal-poor GCs will tend to be concentrated toward the center of the overdensity and will quickly agglomerate into larger structures. Similar mass fluctuations in the less-overdense outer regions will tend to be accreted into larger structures more slowly. Some may survive to form more stars and become dwarf satellites of the central galaxy. This picture, at least as it relates to dark matter halos, is well-understood and accepted. But the important point for GC formation scenarios is that these surviving dwarfs are not representative of the halos that merged to form the central galaxy. The latter collapsed first and may have very different star (and GC) formation histories from those that collapsed later.

This process will operate on a variety of scales. For example, the dwarf satellites of the Galaxy have metal-poor GCs with lower metallicities than those of halo GCs in the Galaxy. Moreover, the disk or E galaxies that merged to form gEs like M87 and NGC 4472 must have had metal-poor GCs with metallicities higher than those typical for Es and spirals in the Virgo cluster today. The metalpoor GCs, although they formed at very high redshift, already "knew" to which galaxy they would ultimately belong. The metal-poor relation rules out merger and accretion models, but only in the local universe for structure forming at the present day. Nonetheless, it is consistent with hierarchical galaxy formation, and is a strong end constraint for any galaxy formation model. To illustrate biasing, Figure 13 shows two snapshots $(z=12$ and 0$)$ of a high-resolution dark matter simulation of the formation of a $10^{12} M_{\odot}$ galaxy (Diemand et al. 2005; Moore et al. 2006). Low-mass, high- $\sigma$ peaks collapse first in a filamentary structure and end up centrally concentrated in the final galaxy.

\section{Cosmological Formation of Metal-poor Globular Clusters}

The preceding sections suggest that the bulk of GCs formed at high redshift. It then follows that they have enormous promise in a cosmological context. In this section we discuss current ideas on the "cosmological" formation of GCs and how this has shaped our overall view of galaxy formation. In what follows, the term cosmological refers to models in which GCs form in low-mass dark matter halos, before the bulk of their parent galaxy has been assembled.

Soon after CDM cosmology was proposed, Peebles (1984) argued that $10^{8} M_{\odot}$ halos, each hosting a few $\times 10^{6} M_{\odot}$ of gas, would be the first to collapse and form stars in the early universe. He suggested GCs as their progeny, and noted that the halos of these GCs might be stripped without disrupting the cluster itself. This basic idea has been sustained to the present day, though the mechanism is probably limited to metal-poor GCs. Rosenblatt, Faber, \& Blumenthal (1988) refined this scenario by suggesting that metal-poor GCs form in $2.8 \sigma$ halos. This gives a reasonable match to the observed radial distribution of metal-poor Galactic GCs and the mass fraction of metal-poor GCs (with respect to total stellar mass) in a variety of galaxies. The principal problem with this picture -indeed, of GC formation in individual dark matter halos in general - is that the minimum required baryonic collapse factor $(\sim 10$ or more) would produce GCs with more rotation than that observed, unless they form preferentially in very low-spin halos, or some other mechanism acts to remove angular momentum. A speculative solution might be to form the GC in the core of a larger gas cloud, if the "extraneous" material can be stripped later. 
Moore (1996) wrote a short influential paper that described the use of N-body simulations to show that the faint tidal tails observed around some GCs (Grillmair et al. 1995) were inconsistent with the presence of extended dark matter halos, but consistent with the low M/L ratios observed in the central regions (e.g., Illingworth 1976; Pryor et al. 1989). The observed tidal tails around, e.g., Pal 5 (Odenkirchen et al. 2002) demonstrate that there are at least some present-day GCs that lack dark matter. However, this does not prove that all (or even most) GCs are free of extended dark matter halos, nor does it rule out metal-poor GC formation inside halos that are later stripped away.

This latter idea has been developed in a number of papers by Mashchenko \& Sills (2005a,b), who studied the formation and evolution of GCs with individual dark matter halos. With high-resolution N-body simulations, they found that (depending on the details of the actual collapse) many properties of simulated GCs with halos are similar to those of observed GCs. For example, the central mass-to-light ratios are expected to be quite low. Structure in the halo (e.g., triaxiality, or breaks in the outer parts of the density profile) could easily be manifested as tidal cutoffs, extratidal stars, or eccentric outer contours; such features are not incompatible with the presence of dark matter. When a GC with a dark matter halo evolves in a tidal field, their simulations indicated that it loses either most (for an NFW halo) or nearly all (for a Burkert halo) of its dark matter. This finding was confirmed in a very high resolution dark matter and gas simulation by Saitoh et al. (2005).

Bromm \& Clarke (2002) used a simulation with both dark matter and gas to study GC formation at high redshift. As noted by Peebles (1984), $\sim 10^{8} M_{\odot}$ minihalos are expected to collapse out of $3 \sigma$ fluctuations at $z \sim 15$. At a high fixed gas density threshold Bromm \& Clarke created sink particles as "GCs". These GCs initially form inside of halos, but the simultaneous collapse of mass scales results in violent relaxation that erases most of the substructure. The resulting GC mass spectrum is set by that of the dark matter, and is a power law with index $\sim-1.8$. The main problem here is that it is not possible to tell, with the current level of sophistication of their simulations, whether the violent relaxation is real or merely an artifact of insufficient resolution.

Other authors have explored in more detail the triggering mechanism for putative GC formation in dark matter halos. In the model of Cen (2001), ionization fronts from cosmic reionization shock gas in low-mass halos. The gas is compressed by a factor $\sim 100$ and collapses to form metal-poor GCs. To produce the observed numbers of GCs, a large population of low spin $(\lambda<0.01)$ halos is required. This condition appears to be satisfied when the halo number density is modeled with extended Press-Schecter theory, but whether it would hold in highresolution cosmological simulations is unknown. This model predicts a power law GCMF with a slope of $\sim-2$, similar to that observed at the high mass end, and has the rather attractive property of predicting no GC mass-radius relation, consistent with the observations. Côté (2002) pointed out that this picture could predict a large number of (unobserved) intergalactic GCs; this objection might be addressed if the ionization fronts are effective above a threshold only met quite close to protogalaxies.

Scannapieco et al. (2004) proposed a somewhat similar mechanism, in which gas in minihalos is shock-compressed by galaxy outflows. The momentum of the shock strips the gas from the halo, nicely solving the dark matter problem. However, this model predicts a mass-radius relation for individual GCs, and the 
observed lack of such a relation may deal this picture a fatal blow.

Ricotti (2002) suggested that GCs themselves could have reionized the universe. The predicted number of ionizing photons appears to be sufficient, assuming that the escape fraction of such photons is near unity. Such a high escape fraction is qualitatively feasible, given the extended spatial distribution of the GCs with respect to their parent galaxies, but the scenario requires detailed modeling (including radiative transfer) in the proper cosmological setup.

The high-resolution simulation of Kravtsov \& Gnedin (2005) offers a glimpse of what should be possible in the future. They performed a gas and dark matter simulation of the formation of a Milky Way analogue to $z \sim 3$. They were not able to resolve GC formation directly, but assumed GCs formed in the cores of GMCs when the dynamical time exceeded the cooling time. These GMCs were located in the flattened gas disks of protogalaxies. The resulting mass function appears to be consistent with that of massive GCs, but the metallicity distribution does not: at the end of the simulation, large numbers of (unobserved) $[\mathrm{Fe} / \mathrm{H}] \sim-1$ GCs were being formed, even though their simulation included feedback. A desirable future extension of such simulations is to test whether reionization might effectively end metal-poor GC formation where "traditional" stellar feedback cannot.

Moore et al. (2006) have shown that the observed radial distribution of metalpoor GCs in the Galaxy can be reproduced if the GCs are assumed to form in $\gtrsim 2.5 \sigma$ peaks of $\gtrsim 2 \times 10^{8} M_{\odot}$ that collapse and form stars before $z \sim 12$ (see Figure 14). If the key assumption of truncation by reionization is supported by other evidence, then the surface density distributions of metal-poor GCs could be used to probe reionization in a variety of galaxies, setting limits on the epoch and homogeneity of reionization.

In summary, the cosmological formation of metal-poor GCs is supported by several lines of argument. (i) The ages of metal-poor GCs. The absolute ages of GCs are still poorly known, and must be continually revised in light of advances in a variety of subfields (for example, the recent revision of the ${ }^{14} \mathrm{~N}(p, \gamma){ }^{15} \mathrm{O}$ reaction rate increases GC ages by 0.7-1 Gyr; Imbriani et al. 2004). If the GC ages are sufficiently close to the age of the universe, cosmological formation becomes a necessity, since low-mass halos as described above are the only existing sites for star formation. (ii) The recently discovered correlation between GC metallicity and mass for bright metal-poor GCs in several massive galaxies (Strader et al. 2006; Harris et al. 2006). Self-enrichment is a potential explanation for this correlation, and it is possible that metals could only be retained in the potential well of a dark matter halo. (iii) The radial distribution of metal-poor GCs. Moore et al. (2006) show that the metal-poor GCs in the Galaxy have a radial distribution consistent with formation in $2.5 \sigma$ peaks in the dark matter distribution at $z>12$. (iv) Observations of Local Group dwarfs show that the lowest-mass galaxies with GCs have total masses of $\lesssim 10^{8} M_{\odot}$ (see $\S 10$ ), as expected under cosmological formation.

Does this mean that two separate mechanisms are needed to explain metalpoor and metal-rich GC formation? The strongest similarity between the two subpopulations is their mass function; for the less evolved high-mass part of the mass function, these are approximately power laws with indices $\sim-1.8$ to -2 . Since power law distributions are a consequence of a variety of physical processes, this similarity does not mandate an identical formation history for both subpopulations. For example, the power law slope observed in the GC mass function is the same as both that of GMCs in the Galaxy and of low-mass dark 
matter halos collapsing at $z \sim 15-20$.

\section{$13 \quad$ Future Directions}

Those of us actively trying to understand extragalactic globular clusters and their connection to galaxy formation are currently operating in a data-dominated rather than a theory-dominated field. We are accumulating a wealth of observational information that we cannot fully interpret. An urgent need exists for improvements in numerical and semi-analytic simulations to help identify GC formation sites and track their spatial, kinematic, chemical, and structural evolution. Models that can resolve the masses and sizes of a typical GC are tantalizingly close to implementation, and their advent will signal a leap in progress toward placing the formation of GCs in its proper cosmological context. With the new generation of "big telescope" wide-field multiplexing spectrographs, such as Keck/DEIMOS, Magellan/IMACS, MMT/Hectospec, and VLT/VIMOS, it is possible to study large samples of GCs in a wide variety of galaxies to carry out detailed tests of these future models, establishing ages, metallicities, and kinematics.

Other important developments will come from the community of SSP modelers. As already mentioned, there remain significant disagreements within this community on the treatment of $\alpha$-enhancement, horizontal branch morphology (the second parameter problem), and underlying stellar synthesis techniques. A new way forward to study extragalactic GCs in detail is the SSP modeling of high-resolution spectra (e.g., Bernstein \& McWilliam 2005). This offers the possibility of estimating abundances of light, $\alpha$-, Fe-peak, and even some strong rand s-process elements. With an 8-10m class telescope, this technique can be applied to significant samples of GCs in the Local Group and nearby galaxies, and even to the brightest few GCs in Virgo. This program is in its infancy, but could represent a significant leap in our understanding of the detailed formation histories of galaxies. GCs may offer the only route to measuring the abundances of interesting elements that are unobservable in massive galaxies themselves due to their large velocity dispersions. A possibility enabled by multiplexing highresolution spectroscopy is detailed dynamical modeling of individual Galactic GCs, to determine whether any contain halo dark matter. The discovery of dark matter in GCs would be a "smoking gun" of cosmological GC formation.

With the development of new large-format detectors and CCD mosaics, wide field optical imaging is poised to address numerous outstanding issues. Obtaining global radial and color distributions for individual GC subpopulations is essential to test scenarios for GC and galaxy formation, especially as models emerge that predict these quantities in detail. Such imaging can also be used to probe the evolution of the GCLF, and provide a definitive test of the scenario in which GCs are formed with a power-law LF that subsequently evolves through various destruction processes to the log-normal LF observed for old GC systems. Since such processes are expected to operate more efficiently at small galactocentric radii, changes in the GCLF of GCs with galactocentric radius would be revealing. Such imaging has the potential to constrain the epoch of reionization from the spatial distribution of metal-poor GCs (as discussed in $§ 12$ ).

These ideas cover only a small fraction of the important advances likely to occur in the field over the next decade. The eventual availability of $30-\mathrm{m}$ class 
telescopes and JWST may allow us to reach the inspiring goal of Renzini (2002): "To directly map the evolution of GCs in galaxies all the way to see them in formation, and eventually stick on the wall a poster with a million-pixel picture of a $z=5$ galaxy, with all her young GCs around."

We thank many colleagues for reading drafts of this manuscript and for useful discussions, including Keith Ashman, Michael Beasley, Javier Cenarro, Laura Chomiuk, Juerg Diemand, Sandra Faber, Duncan Forbes, Genevieve Graves, Soeren Larsen, John Kormendy, Piero Madau, Joel Primack, Katherine Rhode, Tom Richtler, Brad Whitmore, and Steve Zepf. We also thank Michael Beasley, Andres Jordán, and Tom Richtler for permission to use their figures. Michael Beasley, Soeren Larsen, and Katherine Rhode provided data used to create other figures. Finally, we thank Takayuki Saitoh and Marsha Wolf for providing advance copies of their articles. Support was provided by NSF Grants AST-0206139 and AST-0507729, an NSF Graduate Research Fellowship, and STScI grant GO9766 . 


\begin{tabular}{|c|c|c|c|c|c|c|c|c|c|}
\hline Name & Galaxy Type ${ }^{a}$ & Environment ${ }^{b}$ & $\begin{array}{l}M_{B}{ }^{c} \\
(\mathrm{mag}) \\
\end{array}$ & $\underset{(\mathrm{mag})}{\mathrm{MP} \text { colord }^{d}}$ & $\underset{(\mathrm{mag})}{\mathrm{MR} \text { color }} d$ & $\underset{(\mathrm{dex})}{\operatorname{Me} / \mathrm{H}]}{ }^{e}$ & $\underset{(\mathrm{dex})}{\mathrm{MR}[\mathrm{Fe} / \mathrm{H}]^{e}}$ & Color $f$ & $S_{N}{ }^{g}$ \\
\hline $\begin{array}{l}\text { NGC } 4472 \\
\text { nGC }\end{array}$ & core $\mathrm{E}$ & 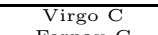 & -21.9 & 0.951 & $\begin{array}{ll}1.411 \\
.485\end{array}$ & $\begin{array}{ll}-1.32 \\
\end{array}$ & $\begin{array}{ll}-0.17 \\
\end{array}$ & $\overline{g-z}$ & 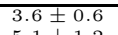 \\
\hline NGC 1399 & core $\mathrm{E}$ & Fornax C & -21.8 & 0.952 & 1.185 & -1.37 & -0.39 & $V-I$ & $5.1 \pm 1.2$ \\
\hline NGC 3309 & core(?) $\mathrm{E}$ & Hydra C & -21.6 & 0.947 & 1.134 & -1.39 & -0.60 & $V-I$ & \\
\hline $\begin{array}{l}\text { NGC } 4486 \\
\text { nat }\end{array}$ & core $\mathrm{E}$ & Virgo C & -21.5 & 0.953 & 1.390 & -1.31 & -0.21 & $g-z$ & $14.1 \pm 1.5$ \\
\hline $\begin{array}{l}\text { NGC } 3311 \\
\text { NGC } 4406\end{array}$ & $\begin{array}{l}\text { core(?) } \mathrm{E} \\
\text { core } \mathrm{E}\end{array}$ & $\begin{array}{l}\text { Hydra C } \\
\text { Virgo C }\end{array}$ & $\begin{array}{l}-21.5 \\
-21.5\end{array}$ & $\begin{array}{l}0.929 \\
0.986\end{array}$ & & $\begin{array}{l}-1.47 \\
-1.23\end{array}$ & & $\begin{array}{l}V-I \\
V-I\end{array}$ & \\
\hline $\begin{array}{l}\text { NGC } 4466 \\
\text { NGC } 4649\end{array}$ & $\begin{array}{l}\text { core } \mathrm{E} \\
\text { core E }\end{array}$ & $\begin{array}{l}\text { Virgo C } \\
\text { Virgo C }\end{array}$ & $\begin{array}{l}-21.5 \\
-21.4\end{array}$ & $\begin{array}{l}0.986 \\
0.964\end{array}$ & $\begin{array}{l}1.145 \\
1.424\end{array}$ & $\begin{array}{l}-1.23 \\
-1.26\end{array}$ & $\begin{array}{l}-0.56 \\
-0.14\end{array}$ & $\begin{array}{l}V-I \\
q-z\end{array}$ & $\begin{array}{l}3.5 \pm 0.5 \\
4.1 \pm 1.0\end{array}$ \\
\hline NGC 524 & So & N524 G & $\begin{array}{l}-21.4 \\
-21.4\end{array}$ & $\begin{array}{l}0.964 \\
0.980\end{array}$ & $\begin{array}{l}1.424 \\
1.189\end{array}$ & $\begin{array}{l}-1.26 \\
-1.25\end{array}$ & $\begin{array}{l}-0.14 \\
-0.37\end{array}$ & $\begin{array}{l}g-z \\
V-I\end{array}$ & $4.1 \pm 1.0$ \\
\hline NGC 4374 & core $\mathrm{E}$ & Virgo C & -21.2 & 0.927 & 1.322 & -1.45 & -0.33 & $g-z$ & $1.6 \pm 0.3$ \\
\hline NGC 5322 & core(?) $\mathrm{E}$ & $\mathrm{N} 5322 \mathrm{G}$ & -21.2 & 0.942 & & -1.41 & & $V-I$ & \\
\hline $\begin{array}{l}\text { NGC } 4594 \\
\text { NG65 }\end{array}$ & So/Sa & N4594 G & -21.2 & 0.939 & 1.184 & -1.43 & -0.39 & $V-I$ & $2.1 \pm 0.3$ \\
\hline $\begin{array}{l}\text { NGC } 4365 \\
\text { NGC } 7619\end{array}$ & core E & Virgo C & -21.1 & 0.891 & 1.232 & -1.63 & -0.50 & $g-z$ & $\cdots$ \\
\hline $\begin{array}{l}\text { NGC } 7619 \\
\text { NGC } 7562\end{array}$ & $\begin{array}{l}\text { core(?) } \mathrm{E} \\
\text { corre(?) } \mathrm{E}\end{array}$ & $\begin{array}{l}\text { Pegasus C } \\
\text { Pegasus C }\end{array}$ & $\begin{array}{l}-21.1 \\
-20.9\end{array}$ & 0.973 & $\ldots$ & $\begin{array}{l}-1.28 \\
-1.51\end{array}$ & & $\begin{array}{l}V-I \\
V-I\end{array}$ & \\
\hline $\begin{array}{l}\text { NGC } 7562 \\
\text { NGC } 2768\end{array}$ & $\begin{array}{c}\text { core(?) } \\
\text { SO }\end{array}$ & $\begin{array}{l}\text { Pegasus C } \\
\text { N2768 G }\end{array}$ & $\begin{array}{l}-20.9 \\
-20.8\end{array}$ & $\begin{array}{l}0.920 \\
0.919\end{array}$ & $\cdots$ & $\begin{array}{l}-1.51 \\
-1.51\end{array}$ & $\cdots$ & $\begin{array}{l}V-I \\
V-I\end{array}$ & $\ldots$ \\
\hline NGC 4621 & transition $\mathrm{E}$ & Virgo C & -20.7 & 0.927 & 1.305 & -1.45 & -0.36 & $g-z$ & \\
\hline NGC 5813 & core $\mathrm{E}$ & N5846 G & -20.6 & 0.935 & & -1.44 & $\ldots$ & $V-I$ & $5.7 \pm 1.8$ \\
\hline $\begin{array}{l}\text { IC } 1459 \\
\text { NGC } 3115\end{array}$ & core E & I1459 G & -20.6 & 0.955 & & -1.36 & & $V-I$ & $\cdots$ \\
\hline $\begin{array}{l}\text { NGC } 3115 \\
\text { NGC } 4494\end{array}$ & $\begin{array}{l}\text { So } \\
\text { power }\end{array}$ & $\begin{array}{l}\text { N3115 G } \\
\text { N4565 G }\end{array}$ & $\begin{array}{l}-20.4 \\
-20.4\end{array}$ & 0.922 & $\begin{array}{l}1.153 \\
1.128\end{array}$ & $\begin{array}{l}-1.50 \\
-1.59\end{array}$ & -0.52 & $V-I$ & $\cdots$ \\
\hline $\begin{array}{l}\text { NGC } 4944 \\
\text { NGC } 4552\end{array}$ & $\begin{array}{l}\text { power E } \\
\text { core E }\end{array}$ & $\begin{array}{l}\text { N4565 G } \\
\text { Virgo C }\end{array}$ & $\begin{array}{l}-20.4 \\
-20.3\end{array}$ & $\begin{array}{l}0.901 \\
0.951\end{array}$ & $\begin{array}{l}1.128 \\
1.334\end{array}$ & $\begin{array}{l}-1.59 \\
-1.32\end{array}$ & $\begin{array}{l}-0.63 \\
-0.31\end{array}$ & $\begin{array}{l}V-1 \\
g-z\end{array}$ & $\cdots$ \\
\hline NGC 253 & $\mathrm{Sc}$ & Sculptor G & -20.3 & 0.912 & 1.334 & -1.54 & $\ldots$ & $\begin{array}{l}g-z \\
V-I\end{array}$ & $\cdots$ \\
\hline NGC 1404 & core(?) E & Fornax C & -20.3 & 0.938 & 1.170 & -1.43 & -0.45 & $V-I$ & $2 \pm 0.5$ \\
\hline M31 & & Local G & -20.2 & 0.912 & & -1.54 & & $V-I$ & 1.3 \\
\hline NGC 3379 & core $\mathrm{E}$ & Leo I G & -20.1 & 0.964 & 1.167 & -1.32 & -0.47 & $V-I$ & $1.2 \pm 0.3$ \\
\hline NGC 4278 & core $\mathrm{E}$ & $\mathrm{N} 4631 \mathrm{G}$ & -20.1 & 0.908 & & -1.56 & & $V-I$ & \\
\hline NGC 4473 & power $\mathrm{E}$ & Virgo C & -20.0 & 0.942 & 1.310 & -1.37 & -0.35 & $g-z$ & .. \\
\hline NGC 3608 & core $\mathrm{E}$ & N3607 G & -20.0 & 0.923 & & -1.49 & & $V-I$ & $\cdots$ \\
\hline NGC 1400 & so & Eridanus G & -19.9 & 0.951 & 1.164 & -1.38 & -0.48 & $V-I$ & \\
\hline Milky Way & Sbc & Local G & -19.9 & 0.898 & & -1.60 & & $V-I$ & 0.7 \\
\hline $\begin{array}{l}\text { NGC } 1023 \\
\text { NGC }\end{array}$ & So & N1023 G & -19.7 & 0.912 & 1.164 & -1.54 & -0.48 & $V-I$ & $\cdots$ \\
\hline $\begin{array}{l}\text { NGC } 4291 \\
\text { NGC } 3384\end{array}$ & core $\mathrm{E}$ & $\mathrm{N} 4291 \mathrm{G}$ & -19.6 & 0.940 & & -1.42 & & $V-I$ & $\cdots$ \\
\hline $\begin{array}{l}\text { NGC } 3384 \\
\text { NGC } 3607\end{array}$ & $\begin{array}{l}\text { So } \\
\text { so }\end{array}$ & $\begin{array}{l}\text { Leo I G } \\
\text { N3607 G }\end{array}$ & $\begin{array}{l}-19.5 \\
-19.5\end{array}$ & $\begin{array}{l}0.942 \\
0.939\end{array}$ & $\begin{array}{l}1.208 \\
1.099\end{array}$ & $\begin{array}{l}-1.41 \\
-1.43\end{array}$ & $\begin{array}{l}-0.29 \\
-0.75\end{array}$ & $\begin{array}{l}V-I \\
V-I\end{array}$ & $\cdots$ \\
\hline $\begin{array}{l}\text { NGC } 3607 \\
\text { NGC } 1427\end{array}$ & $\begin{array}{l}\text { So } \\
\text { power E }\end{array}$ & $\begin{array}{l}\text { N3607 G } \\
\text { Fornax C }\end{array}$ & $\begin{array}{l}-19.5 \\
-19.4\end{array}$ & $\begin{array}{l}0.939 \\
0.940\end{array}$ & $\begin{array}{l}1.099 \\
1.153\end{array}$ & $\begin{array}{l}-1.43 \\
-1.42\end{array}$ & $\begin{array}{l}-0.75 \\
-0.52\end{array}$ & $\begin{array}{l}V-I \\
V-I\end{array}$ & $3.4 \pm 0.6$ \\
\hline NGC 4478 & power E & Virgo C & -19.2 & 0.882 & 1.195 & -1.68 & -0.56 & $g-z$ & \\
\hline NGC 4434 & power E & Virgo C & -19.2 & 0.911 & 1.179 & -1.53 & -0.59 & $g-z$ & $\cdots$ \\
\hline $\begin{array}{l}\text { NGC } 3377 \\
\text { NGC }\end{array}$ & power $\mathrm{E}$ & Leo I G & -19.2 & 0.936 & 1.103 & -1.44 & -0.74 & $V-I$ & \\
\hline $\begin{array}{l}\text { NGC } 4564 \\
\text { NGC } 4387\end{array}$ & $\begin{array}{l}\text { So } \\
\text { ower F }\end{array}$ & $\begin{array}{l}\text { Virgo C } \\
\text { Virgo C }\end{array}$ & $\begin{array}{l}-19.0 \\
-19.0\end{array}$ & 0.935 & 1.263 & $\begin{array}{l}-1.40 \\
-1.79\end{array}$ & -0.44 & $g-z$ & $\cdots$ \\
\hline $\begin{array}{l}\text { NGC } 4387 \\
\text { NGC } 4660\end{array}$ & $\begin{array}{l}\text { power E } \\
\text { So }\end{array}$ & $\begin{array}{l}\text { Virgo C } \\
\text { Virgo C }\end{array}$ & $\begin{array}{l}-19.0 \\
-18.7\end{array}$ & $\begin{array}{l}0.859 \\
0.923\end{array}$ & $\begin{array}{l}1.112 \\
1.320\end{array}$ & $\begin{array}{l}-1.79 \\
-1.47\end{array}$ & $\begin{array}{l}-0.72 \\
-0.33\end{array}$ & $\begin{array}{l}g-z \\
g-z\end{array}$ & $\ldots$ \\
\hline NGC 247 & $\mathrm{Sd}$ & Sculptor G & -18.7 & 0.908 & & -1.56 & -0.00 & $V-I$ & $\ldots$ \\
\hline NGC 4733 & power E & Virgo C & -18.6 & 0.918 & 1.131 & -1.52 & -0.62 & $V-I$ & \\
\hline NGC 4550 & so & Virgo C & -18.6 & 0.883 & 1.145 & -1.66 & -0.56 & $V-I$ & $\cdots$ \\
\hline NGC 4489 & So & Virgo C & -18.5 & 0.900 & 1.260 & -1.58 & -0.44 & $g-z$ & $\cdots$ \\
\hline NGC 4551 & power E & Virgo C & -18.5 & 0.890 & 1.219 & -1.64 & -0.52 & $g-z$ & $\cdots$ \\
\hline M33 & Scd & Local G & -18.4 & 0.900 & & -1.59 & 0.02 & $V-I$ & $\ldots$ \\
\hline NGC 4458 & power $\mathrm{E}$ & Virgo C & -18.4 & 0.892 & 1.223 & -1.63 & -0.51 & $g-z$ & \\
\hline NGC 55 & $\mathrm{Sm}$ & Sculptor G & -18.3 & 0.892 & & -1.63 & & $V-I$ & \\
\hline IC 3468 & $\mathrm{dE}$ & Virgo C & -18.1 & 0.925 & 1.130 & -1.46 & -0.68 & $g-z$ & 1.1 \\
\hline $\begin{array}{l}\text { NGC } 300 \\
\text { NGC } 4482\end{array}$ & $\mathrm{Sd}$ & Sculptor G & -18.1 & 0.892 & & -1.63 & 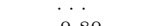 & $V-I$ & \\
\hline $\begin{array}{l}\text { NGC } 4482 \\
\text { LMC }\end{array}$ & $\mathrm{dE}$ & Virgo C & -18.1 & 0.884 & 1.065 & -1.67 & -0.80 & $g-z$ & 1.6 \\
\hline $\begin{array}{l}\text { LMC } \\
\text { NGC } 3599\end{array}$ & $\begin{array}{l}\text { dirr } \\
\text { So }\end{array}$ & $\begin{array}{l}\text { Local G } \\
\text { Leo I G }\end{array}$ & $\begin{array}{l}-18.0 \\
-17.6\end{array}$ & $\begin{array}{l}0.890 \\
0.872\end{array}$ & 1.112 & $\begin{array}{l}-1.63 \\
-1.71\end{array}$ & -0.70 & $\begin{array}{l}V-I \\
V-I\end{array}$ & 0.8 \\
\hline IC 3019 & $\mathrm{dE}$ & Virgo C & -17.2 & 0.864 & $\begin{array}{l}1.112 \\
1.093\end{array}$ & -1.77 & -0.75 & $g-z$ & 1.8 \\
\hline IC 3381 & $\mathrm{dE}$ & Virgo $\mathrm{C}$ & -17.1 & 0.897 & 1.168 & -1.60 & -0.61 & $g-z$ & 5.2 \\
\hline IC 3328 & $\mathrm{dE}$ & Virgo $\mathrm{C}$ & -17.1 & 0.905 & 1.114 & -1.56 & -0.71 & $g-z$ & 0.9 \\
\hline $\begin{array}{l}\text { NGC } 4318 \\
\text { SC } 89\end{array}$ & $\mathrm{dE}$ & $\begin{array}{l}\text { Virgo C } \\
\text { Vireco }\end{array}$ & -17.1 & 0.884 & 1.182 & -1.67 & -0.59 & $g-z$ & 0.7 \\
\hline IC 809 & $\mathrm{dE}$ & Virgo C & -17.1 & 0.864 & 1.129 & -1.77 & -0.68 & $g-z$ & 3.3 \\
\hline $\begin{array}{l}\text { IC } 3653 \\
\text { IC } 3652\end{array}$ & $\begin{array}{l}\text { power E } \\
\mathrm{dE}\end{array}$ & $\begin{array}{l}\text { Virgo C } \\
\text { Virgo C }\end{array}$ & $\begin{array}{l}-17.1 \\
-16.9\end{array}$ & $\begin{array}{l}0.851 \\
0.879\end{array}$ & $\begin{array}{l}\mathrm{N} \\
173\end{array}$ & $\begin{array}{l}-1.84 \\
-1.69\end{array}$ & $\begin{array}{l}\mathrm{N} \\
-060\end{array}$ & $\begin{array}{l}g-z \\
q-z\end{array}$ & $\begin{array}{l}0.6 \\
3.7\end{array}$ \\
\hline VCC 543 & $\mathrm{dE}$ & Virgo C & -16.9 & 0.794 & $\mathrm{~N}$ & -2.13 & $\mathrm{~N}$ & $\begin{array}{l}g-z \\
g-z\end{array}$ & 0.4 \\
\hline IC 3470 & $\mathrm{dE}$ & Virgo $\mathrm{C}$ & -16.9 & 0.938 & 1.138 & -1.39 & -0.67 & $g-z$ & 5.0 \\
\hline NGC $4486 \mathrm{~b}$ & power E & Virgo $\mathrm{C}$ & -16.8 & 0.920 & 1.126 & -1.51 & -0.64 & $V-I$ & \\
\hline IC 3501 & $\mathrm{dE}$ & Virgo C & -16.8 & 0.872 & 1.177 & -1.73 & -0.60 & $g-z$ & 5.3 \\
\hline $\begin{array}{l}\text { IC } 3442 \\
\text { VCC } 437\end{array}$ & $\mathrm{dE}$ & Virgo C & -16.8 & 0.837 & 1.089 & -1.91 & -0.76 & $g-z$ & 0.8 \\
\hline $\begin{array}{l}\text { IC } 37357 \\
\text { IC } 337\end{array}$ & $\begin{array}{l}\mathrm{dE} \\
\mathrm{dE}\end{array}$ & $\begin{array}{l}\text { Virgo C } \\
\text { Virgo C }\end{array}$ & $\begin{array}{l}-16.7 \\
-16.7\end{array}$ & $\begin{array}{l}0.894 \\
0.848\end{array}$ & $\begin{array}{l}1.042 \\
1.198\end{array}$ & $\begin{array}{l}-1.61 \\
-1.85\end{array}$ & $\begin{array}{l}-0.85 \\
-0.56\end{array}$ & $\begin{array}{l}g-z \\
g-z\end{array}$ & $\begin{array}{l}1.1 \\
1.6\end{array}$ \\
\hline SMC & $\mathrm{dIrr}$ & Local G & $\begin{array}{l}-16.7 \\
-16.7\end{array}$ & $\begin{array}{l}0.848 \\
0.919\end{array}$ & & $\begin{array}{l}-1.80 \\
-1.51\end{array}$ & & $\begin{array}{l}g-z \\
V-I\end{array}$ & $\begin{array}{l}1.6 \\
1.2\end{array}$ \\
\hline IC 3032 & $\mathrm{dE}$ & Virgo $\mathrm{C}$ & -16.6 & 0.861 & 1.169 & -1.78 & -0.61 & $g-z$ & 0.9 \\
\hline VCC 200 & $\mathrm{dE}$ & Virgo C & -16.6 & 0.931 & 1.101 & -1.42 & -0.74 & $g-z$ & 0.5 \\
\hline IC 3487 & $\mathrm{dE}$ & Virgo $\mathrm{C}$ & -16.5 & 0.824 & 1.086 & -1.97 & -0.76 & $g-z$ & 1.2 \\
\hline IC 3509 & power $\mathrm{E}$ & Virgo C & -16.4 & 0.869 & 1.071 & -1.74 & -0.79 & $g-z$ & 7.3 \\
\hline VCC 1895 & $\mathrm{dE}$ & Virgo $\mathrm{C}$ & -16.3 & 0.818 & 1.071 & -2.01 & -0.79 & $g-z$ & 1.6 \\
\hline IC 3647 & $\mathrm{dE}$ & Virgo $\mathrm{C}$ & -16.2 & 0.845 & $\mathrm{~N}$ & -1.87 & $\mathrm{~N}$ & $g-z$ & 1.0 \\
\hline IC 3383 & $\mathrm{dE}$ & Virgo $\mathrm{C}$ & -16.2 & 0.834 & 1.056 & -1.92 & -0.82 & $g-z$ & 4.6 \\
\hline VCC 1627 & power E & Virgo $\mathrm{C}$ & -16.2 & 0.755 & $\mathrm{~N}$ & -2.33 & $\mathrm{~N}$ & $g-z$ & 0.5 \\
\hline IC 3693 & power $\mathrm{E}$ & Virgo C & -16.2 & 0.779 & 1.177 & -2.21 & -0.60 & $g-z$ & 1.0 \\
\hline $\begin{array}{l}\text { IC } 3101 \\
\text { IC } 798\end{array}$ & $\frac{\mathrm{dE}}{\mathrm{de}}$ & Virgo C & -16.1 & 0.842 & 1.134 & -1.88 & -0.67 & $g-z$ & 4.9 \\
\hline $\begin{array}{l}\text { IC } 798 \\
\text { IC } 3779\end{array}$ & $\begin{array}{l}\text { power E } \\
d \mathrm{dF}\end{array}$ & $\begin{array}{l}\text { Virgo C } \\
\text { Virgo C }\end{array}$ & $\begin{array}{l}-16.1 \\
-16.1\end{array}$ & $\begin{array}{l}0.854 \\
0.880\end{array}$ & $\begin{array}{l}\mathrm{N} \\
1060\end{array}$ & $\begin{array}{l}-1.82 \\
-1.69\end{array}$ & $\begin{array}{l}\mathrm{N} \\
-081\end{array}$ & $\begin{array}{l}g-z \\
q-z\end{array}$ & $\begin{array}{l}4.6 \\
2.0\end{array}$ \\
\hline IC 3635 & $\mathrm{dE}$ & $\begin{array}{l}\text { Virgo C } \\
\text { Virgo C }\end{array}$ & $\begin{array}{l}-16.1 \\
-16.0\end{array}$ & $\begin{array}{l}0.880 \\
0.898\end{array}$ & $\begin{array}{c}1.060 \\
\mathrm{~N}\end{array}$ & $\begin{array}{l}-1.69 \\
-1.59\end{array}$ & $\begin{array}{c}-0.81 \\
\mathrm{~N}\end{array}$ & $\begin{array}{l}g-z \\
g-z\end{array}$ & $\begin{array}{l}2.0 \\
5.0\end{array}$ \\
\hline VCC 1993 & $\mathrm{dE}$ & Virgo C & -16.0 & 0.851 & $\mathrm{~N}$ & -1.84 & $\mathrm{~N}$ & $g-z$ & 0.3 \\
\hline IC 3461 & $\mathrm{dE}$ & Virgo $\mathrm{C}$ & -15.8 & 0.919 & 1.142 & -1.49 & -0.66 & $g-z$ & 12.1 \\
\hline VCC 1886 & $\mathrm{dE}$ & Virgo C & -15.8 & 0.877 & $\mathrm{~N}$ & -1.70 & $\mathrm{~N}$ & $g-z$ & 1.5 \\
\hline IC 3602 & $\mathrm{dE}$ & Virgo C & -15.7 & 0.855 & $\mathrm{~N}$ & -1.82 & $\mathrm{~N}$ & $g-z$ & 1.1 \\
\hline NGC 205 & $\mathrm{dE}$ & Local G & -15.6 & 0.922 & $\mathrm{~N}$ & -1.50 & $\mathrm{~N}$ & $V-I$ & 3 \\
\hline VCC 1539 & $\mathrm{dE}$ & Virgo C & -15.6 & 0.898 & 1.039 & -1.59 & -0.87 & $g-z$ & 9.5 \\
\hline VCC 1185 & $\mathrm{dE}$ & Virgo C & -15.6 & 0.890 & 1.077 & -1.64 & -0.78 & $g-z$ & 6.3 \\
\hline IC 3633 & $\mathrm{dE}$ & Virgo $\mathrm{C}$ & -15.5 & 0.884 & $\mathrm{~N}$ & -1.67 & $\mathrm{~N}$ & $g-z$ & 3.3 \\
\hline IC 3490 & & Virgo $\mathrm{C}$ & -15.4 & 0.858 & 1.136 & -1.80 & -0.67 & $g-z$ & 7.3 \\
\hline VCC 1661 & $\mathrm{dE}$ & Virgo C & -15.3 & 0.838 & 1.130 & -1.90 & -0.68 & $g-z$ & \\
\hline $\begin{array}{l}\text { NGC } 185 \\
\text { NGC } 6822\end{array}$ & $\begin{array}{l}\mathrm{dE} \\
\mathrm{dIrr}\end{array}$ & $\begin{array}{l}\text { Local G } \\
\text { Local G }\end{array}$ & -14.8 & $\begin{array}{l}0.882 \\
0.850\end{array}$ & $\mathrm{~N}$ & -1.67 & $\mathrm{~N}$ & $V-I$ & 4.6 \\
\hline $\begin{array}{l}\text { NGC } 1472 \\
\text { NGC } 147\end{array}$ & $\underset{d \mathrm{der}}{\mathrm{dir}}$ & $\begin{array}{l}\text { Local G } \\
\text { Local G }\end{array}$ & $\begin{array}{l}-14.7 \\
-14.3\end{array}$ & $\begin{array}{l}0.850 \\
0.807\end{array}$ & $\mathrm{~N}$ & $\begin{array}{l}-1.80 \\
-1.98\end{array}$ & $\mathrm{~N}$ & $\begin{array}{l}V-1 \\
V-I\end{array}$ & $\begin{array}{l}1.2 \\
3.6\end{array}$ \\
\hline WLM & $\mathrm{dIrr}$ & Local G & -13.9 & 0.910 & $\mathrm{~N}$ & -1.55 & $\mathrm{~N}$ & $V-I$ & $\begin{array}{l}3.0 \\
1.7\end{array}$ \\
\hline Sagittarius & $\mathrm{dSph}$ & Local G & -12.8 & 0.871 & & -1.71 & & $V-I$ & 18.1 \\
\hline Fornax & dSph & Local G & -12.6 & 0.858 & $\mathrm{~N}$ & -1.77 & $\mathrm{~N}$ & $V-I$ & 28.8 \\
\hline
\end{tabular}

Table 1: Properties of GC color distributions. 
${ }^{a}$ Power/Core E: Ellipticals with power-law or cored center surface brightness distributions. NGC 4621 is a transition between the two groups. The galaxies with (?) are not formally classified - the division has been made between core/power-law Es at $M_{B}=-20$ (Faber et al. 1997; Kormendy et al. 2006). Classifications are from Kormendy et al. (2006, and private communication) for Virgo galaxies, Faber et al. (1997) for many early-type galaxies, and NED for the remainder. The term $\mathrm{dE}$ (dwarf elliptical) is often used for all non-star forming galaxies with $M_{B} \gtrsim-18$; here we use it only for galaxies with faint central surface brightness and Sersic $\mathrm{n} \sim 1$ (exponential) profiles. ${ }^{b}$ Local galaxy environment. C: cluster, G: group. ${ }^{c}$ References for $M_{B}$ can be found in Strader et al. (2004a) and Strader et al. (2006). ${ }^{d}$ Peak metal-poor (MP) and metal-rich (MR) colors derived from mixture modeling of the GC color distributions. "N" indicates that study of the galay has found no metal-rich GCs. Galaxies with "..." either have metal-rich GCs without a determined color peak (typically massive galaxies), or ambiguous evidence for such a subpopulation. ${ }^{e}[\mathrm{Fe} / \mathrm{H}]$ values converted from the listed colors using the relations of Barmby et al. (2000) and Peng et al. (2006) for $V-I$ and $g-z$, respectively. ${ }^{f}$ The color listed in columns 5 and 6 . The $V-I$ colors are from Strader et al. (2004a); the $g-z$ colors are from Strader et al. (2006) ${ }^{g} V$-band specific frequency $\left(S_{N}\right)$. Values are only listed if from a study with sufficient spatial coverage and photometric depth for an accurate estimate of the total GC population. The values from Strader et al. (2006) have been converted from the $B$-band $S_{N}$ by dividing by 2.1 ; this assumes $B-V=0.8 \cdot{ }^{h}$ Literature sources for $S_{N}$ estimates. 1: Rhode \& Zepf (2004) 2: Dirsch et al. (2003). 3: Harris et al. (1998). 4: Forbes et al. (2004). 5: Gomez \& Richtler (2004). 6: Hopp et al. (1995). 7: Forbes et al. (1998). 8: Forbes et al. (2000). 10: Strader et al. (2006). 11: This work; the $S_{N}$ estimates have been increased from Forbes et al. (2000) to include new GCs in NGC 6822 and Sgr (see text). 


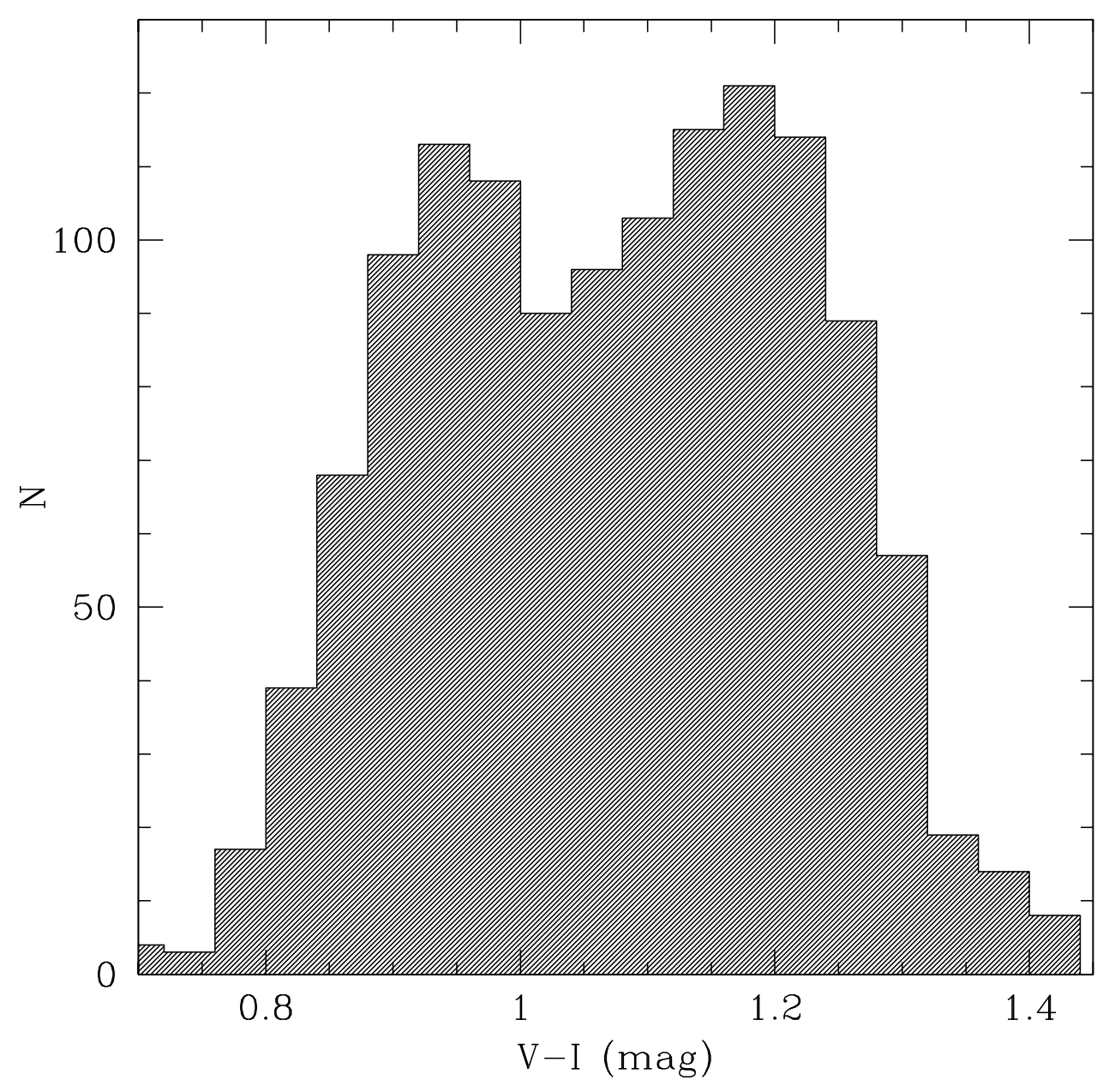

Figure 1: $V-I$ color histogram of GCs in the Virgo gE M87, showing clear bimodality (Larsen et al. 2001; figure from data courtesy of S. Larsen). 


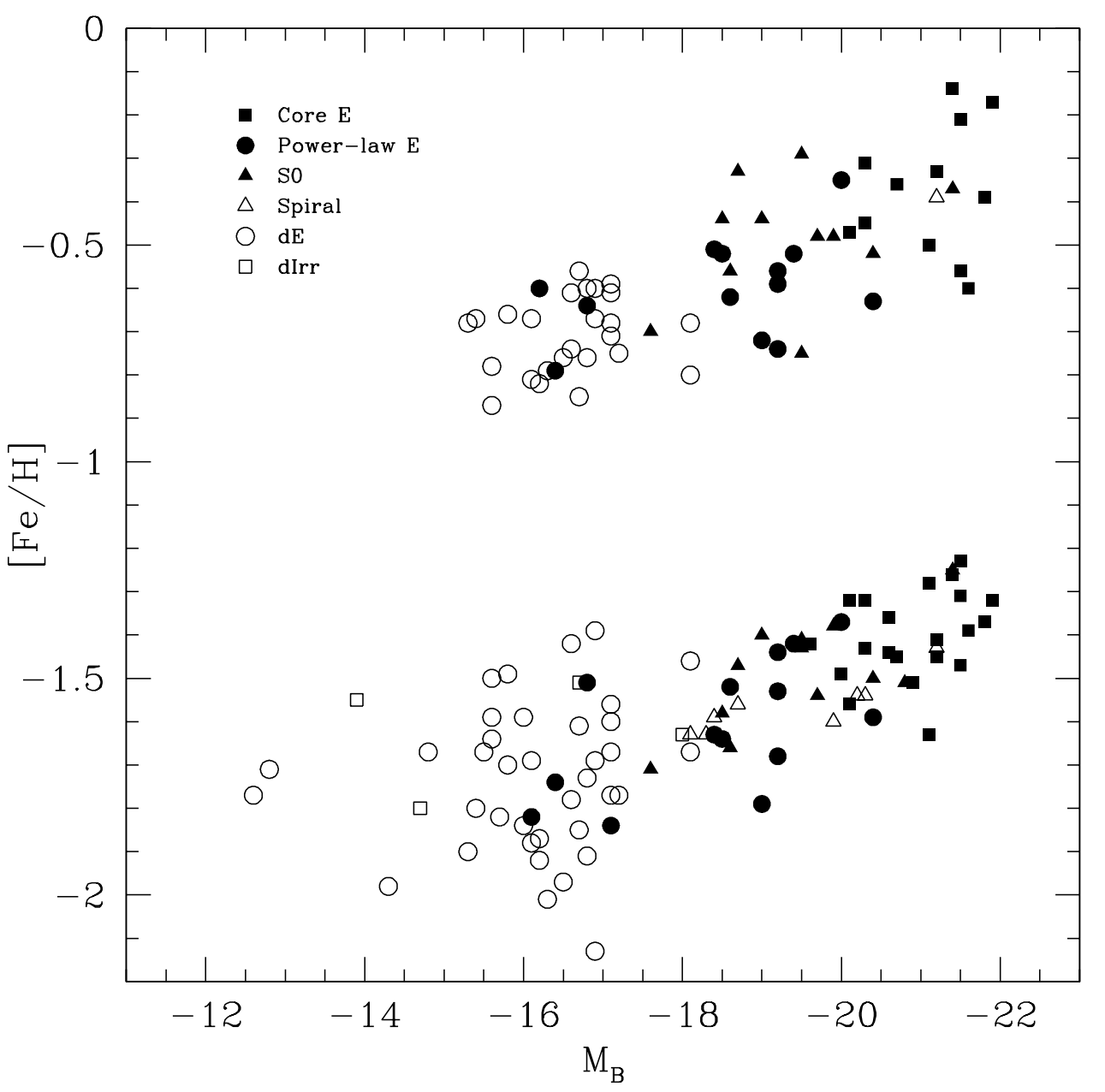

Figure 2: Peak GC metallicity vs. galaxy luminosity $\left(M_{B}\right)$ for metal-poor and metal-rich GCs in a range of galaxies. The points are from Strader et al. (2004a) and Strader et al. (2006) and have been converted from $V-I$ and $g-z$ to $[\mathrm{Fe} / \mathrm{H}]$ using the relations of Barmby et al. (2000) and Peng et al. (2006), respectively. Galaxy types are indicated in the figure key; classifications are in Table 1. Linear relations exist for both subpopulations down to the limit of available data. 


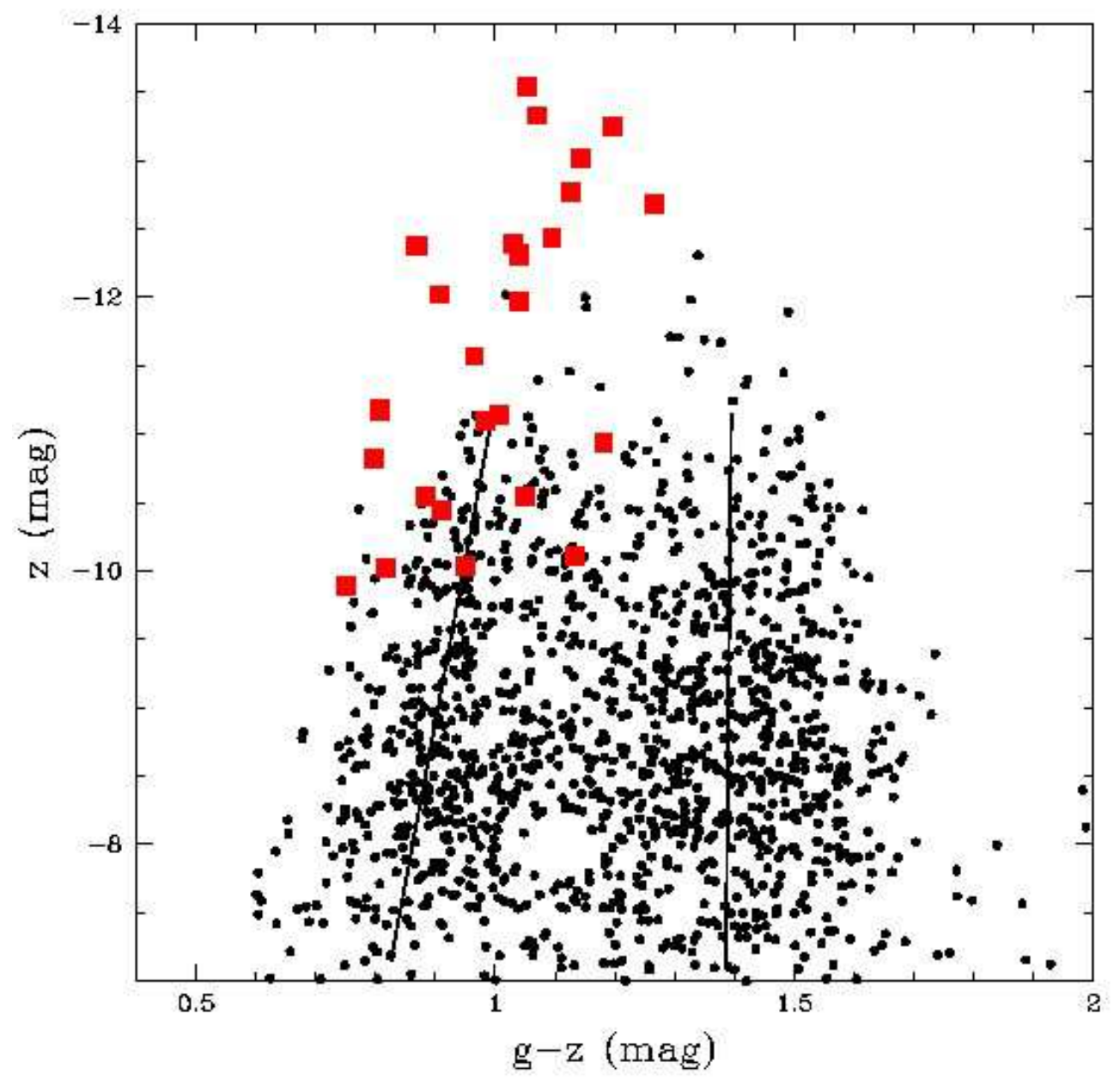

Figure 3: $z$ vs. $g-z$ color-magnitude diagram for M87 GCs (black circles) and Virgo dE nuclei (red squares) from Strader et al. (2006). A correlation between color and luminosity for the bright metal-poor GCs is apparent (the "blue tilt"). The solid lines are fitted linear relations. The $\mathrm{dE}$ nuclei are generally consistent with the sequence of metal-poor GCs, but extend to higher luminosities and have a larger spread in color. 


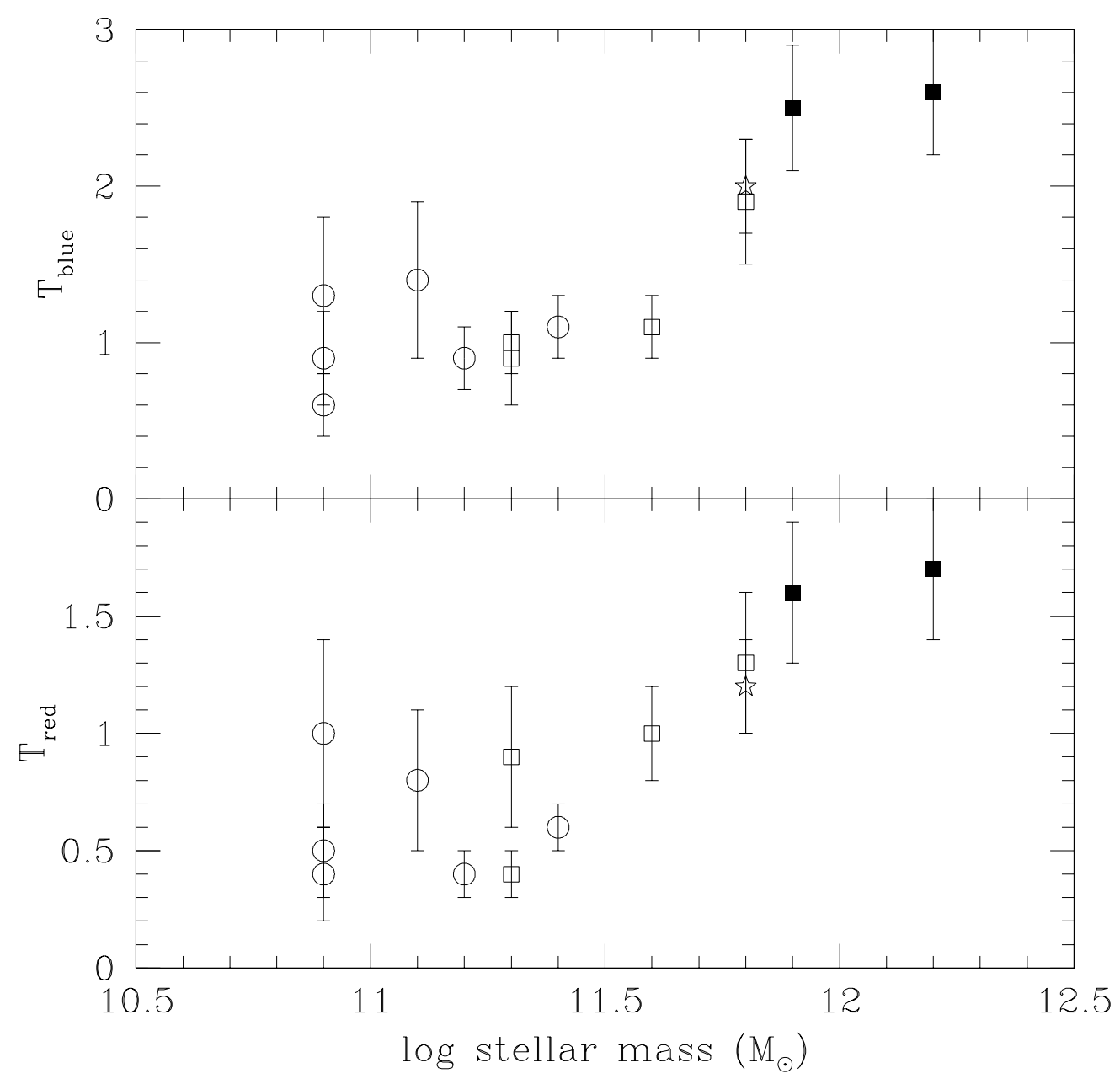

Figure 4: $T_{\text {blue }}$ and $T_{\text {red }}$ (top and bottom panels, respectively) vs. galaxy mass for a range of spirals and Es (Rhode et al. 2005). Filled squares are cluster Es, open squares are field/group Es and S0s, open circles are field/group spirals, and the open star is the Sa/S0 galaxy NGC 4594. There is a general trend of increasing $T_{\text {blue }}$ and $T_{\text {red }}$ with galaxy mass (Data courtesy K. Rhode). 


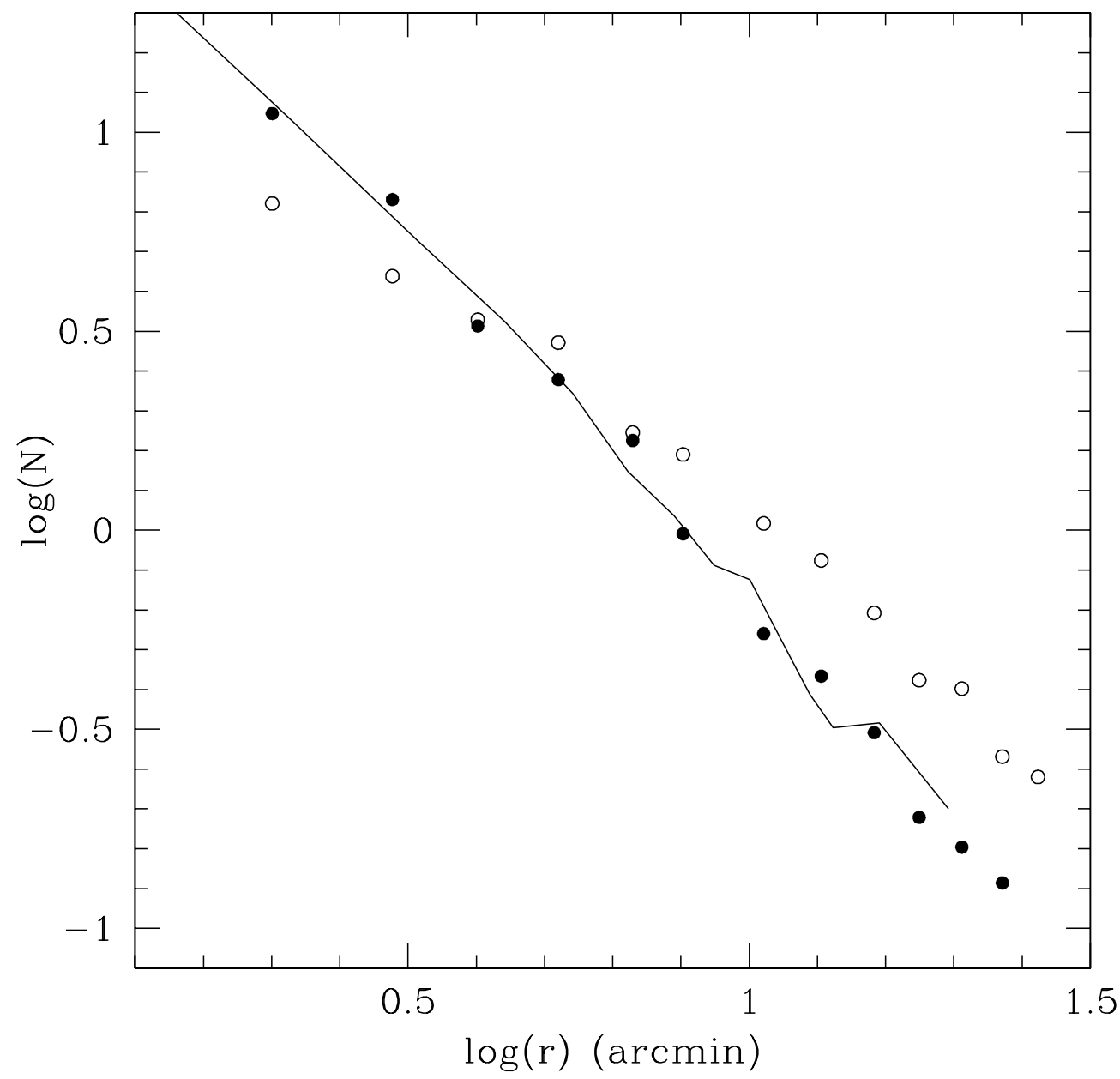

Figure 5: Radial surface density distribution of metal-poor (open circles) and metal-rich (filled circles) GCs in the Fornax gE NGC 1399. The solid line is the scaled galaxy light profile in the $R$-band. The metal-rich GCs are more centrally concentrated, and closely follow the underlying galaxy light. The radial distribution of the metal-poor GCs is flatter; they dominate the GC system at large radii (Bassino et al. 2006). 


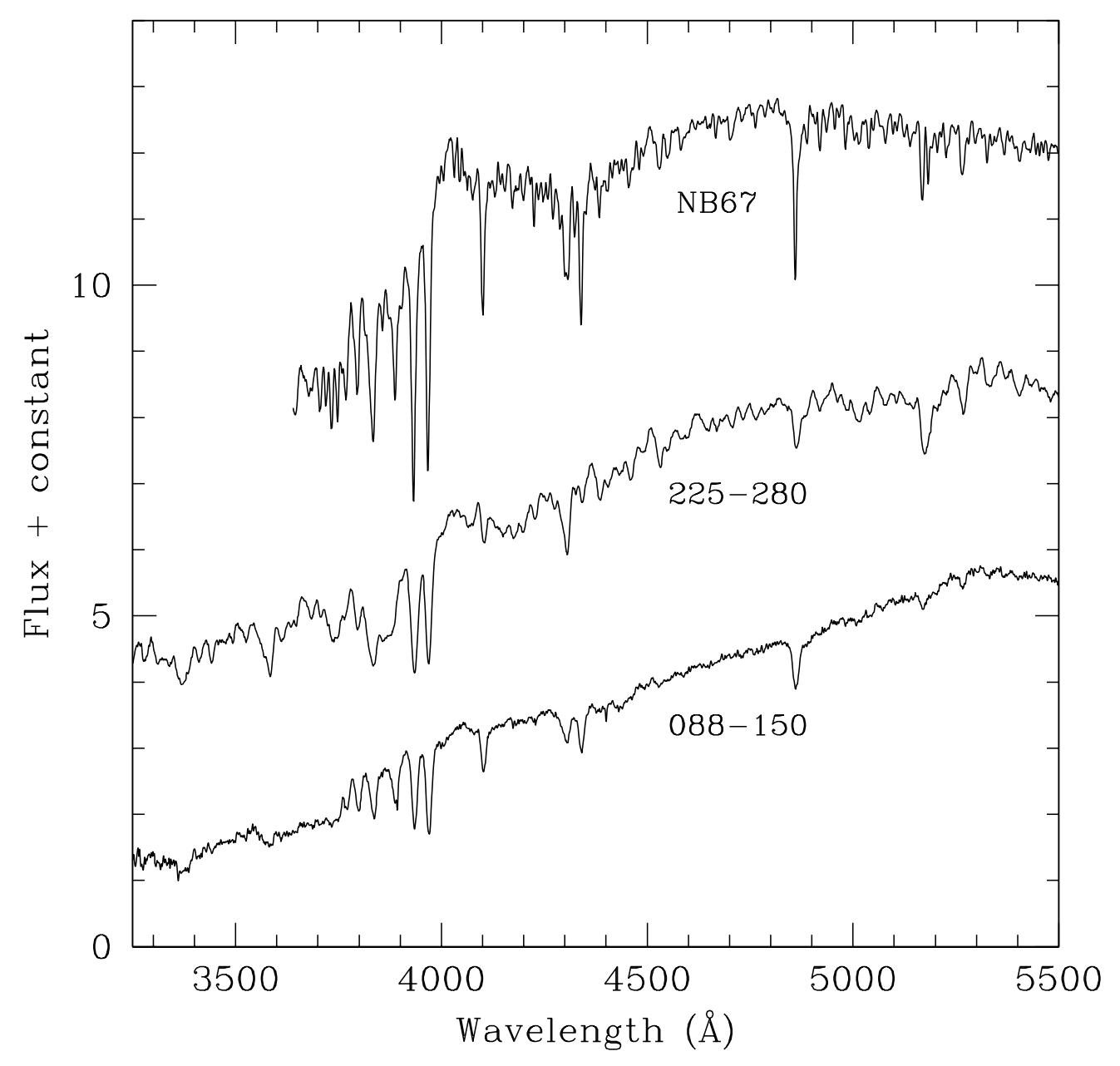

Figure 6: Representative fluxed M31 GC spectra from Keck/LRIS: from bottom to top, an old metal-poor GC (088-150), an old metal-rich GC (225-280), and an intermediate-age, intermediate-metallicity GC (NB67). The spectrum of NB67 is from Beasley et al. (2005), and has been smoothed with a 5-pixel boxcar. The former two spectra are unpublished data of the authors. NB67 has a metallicity $\sim-1$ and an age of $\sim 2$ Gyr; its combination of strong Balmer and metal lines distinguishes it from the old GCs. 


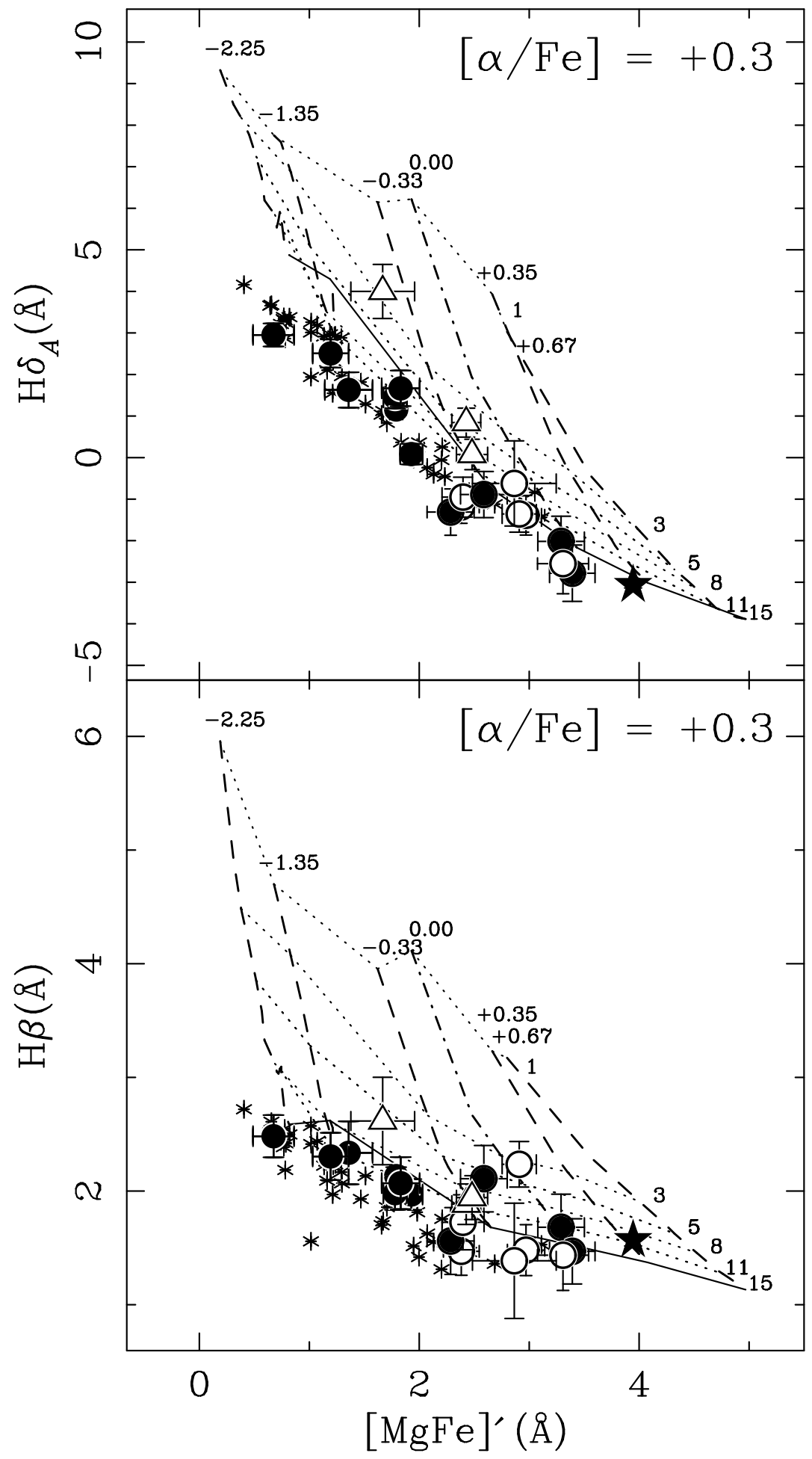

Figure 7: $\mathrm{H} \delta_{A}$ and $\mathrm{H} \beta$ vs. [MgFe]'age-metallicity index-index plots for GCs in NGC 1407 (circles and triangles; Cenarro et al. 2006) and Galactic GCs (stars, from Schiavon et al. 2004). The filled circles are "normal" GCs, the open circles have anomalously high $[\mathrm{Mg} / \mathrm{Fe}]$ and $[\mathrm{C} / \mathrm{Fe}]$, and the open triangles are GCs with enhanced Balmer lines (probably due to blue horizontal branches, but younger ages are also a possibility). The large star is a central $r_{e} / 8$ aperture for NGC 1407 itself. The overplotted grids are Thomas, Maraston, \& Korn (2004) models with $[\alpha / \mathrm{Fe}]=+0.3$ and the ages and metallicities indicated. The model grid lines cross at old ages and low metallicities, making exact age estimates impossible. 


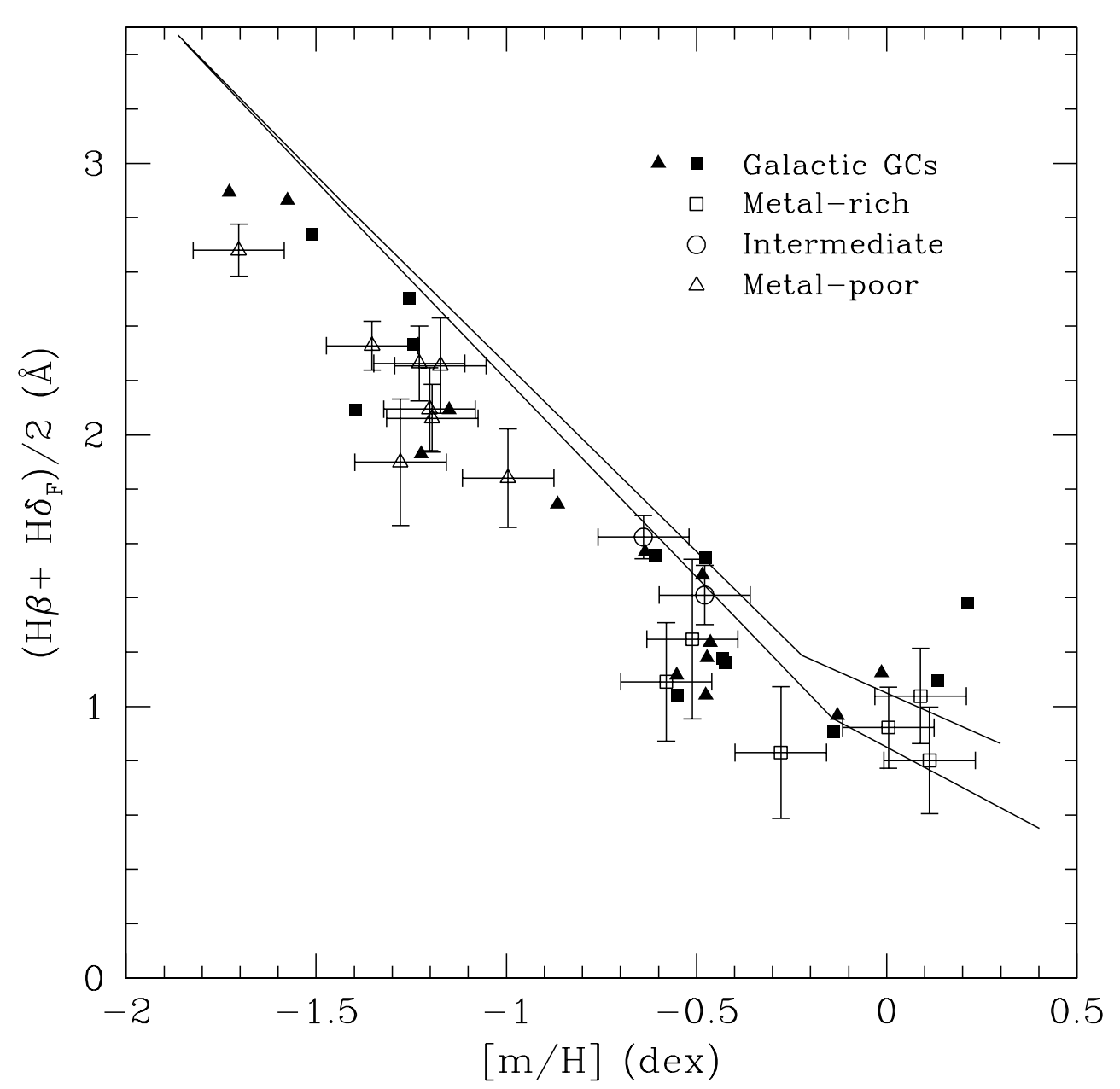

Figure 8: Combined Balmer index vs. metallicity for extragalactic GC subpopulations and Galactic GCs (Strader et al. 2005). The extragalactic subpopulations are plotted as open triangles (metal-poor), open circles (intermediate-metallicity), and open squares (metal-rich), with the subpopulations defined from broadband photometry of the GC systems. Individual Galactic GCs are plotted as filled triangles (data from Gregg 1994) and filled squares (data from Puzia et al. 2002b). At all metallicities, the extragalactic subpopulations appear coeval with (or older than) the comparison Galactic GCs. This suggests mean ages > 10-13 Gyr for the extragalactic GCs. 14 Gyr model lines from Thomas, Maraston, \& Korn (2004) are superposed $([Z / \mathrm{H}]=-2.25$ to 0 , with $[\alpha / \mathrm{Fe}]=0$ on bottom and +0.3 on top). These include a blue horizontal branch below $[Z / \mathrm{H}]=-1.35$. At fixed metallicity, older ages lie at weaker Balmer line strength. The data are not fully calibrated to the models, so cannot be directly compared, and the offset between the data and the model lines is not significant. 

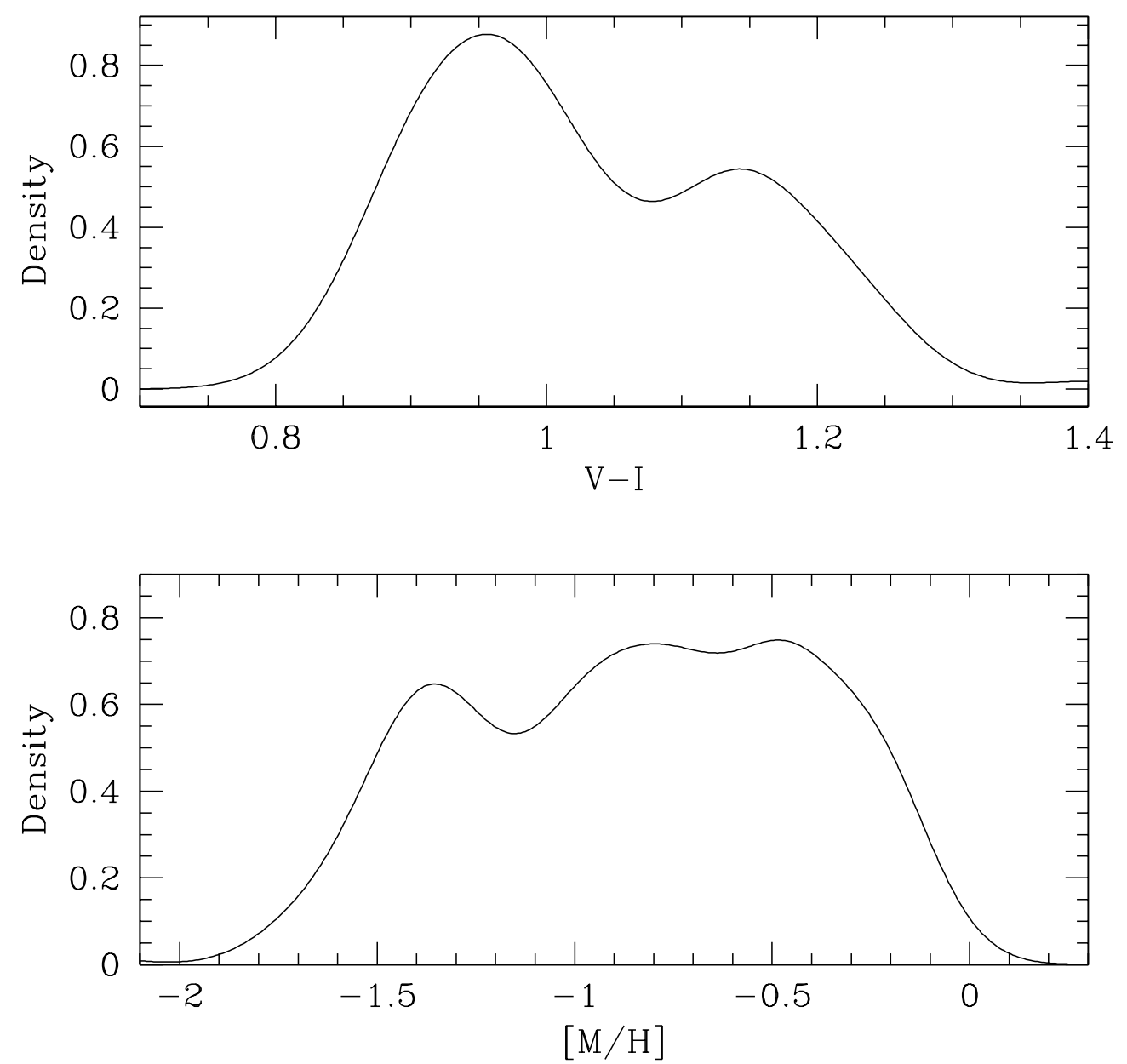

Figure 9: Smoothed kernel density histograms in $V-I$ and $[\mathrm{m} / \mathrm{H}]$ for GCs in NGC 5128. The kernels used are $0.03 \mathrm{mag}$ and 0.1 dex, respectively. The metallicity histogram shows evidence for three distinct subpopulations of GCs, and a comparison of the two histograms indicates the clear nonlinearity of the color-metallicity relation (Beasley et al. 2006). 


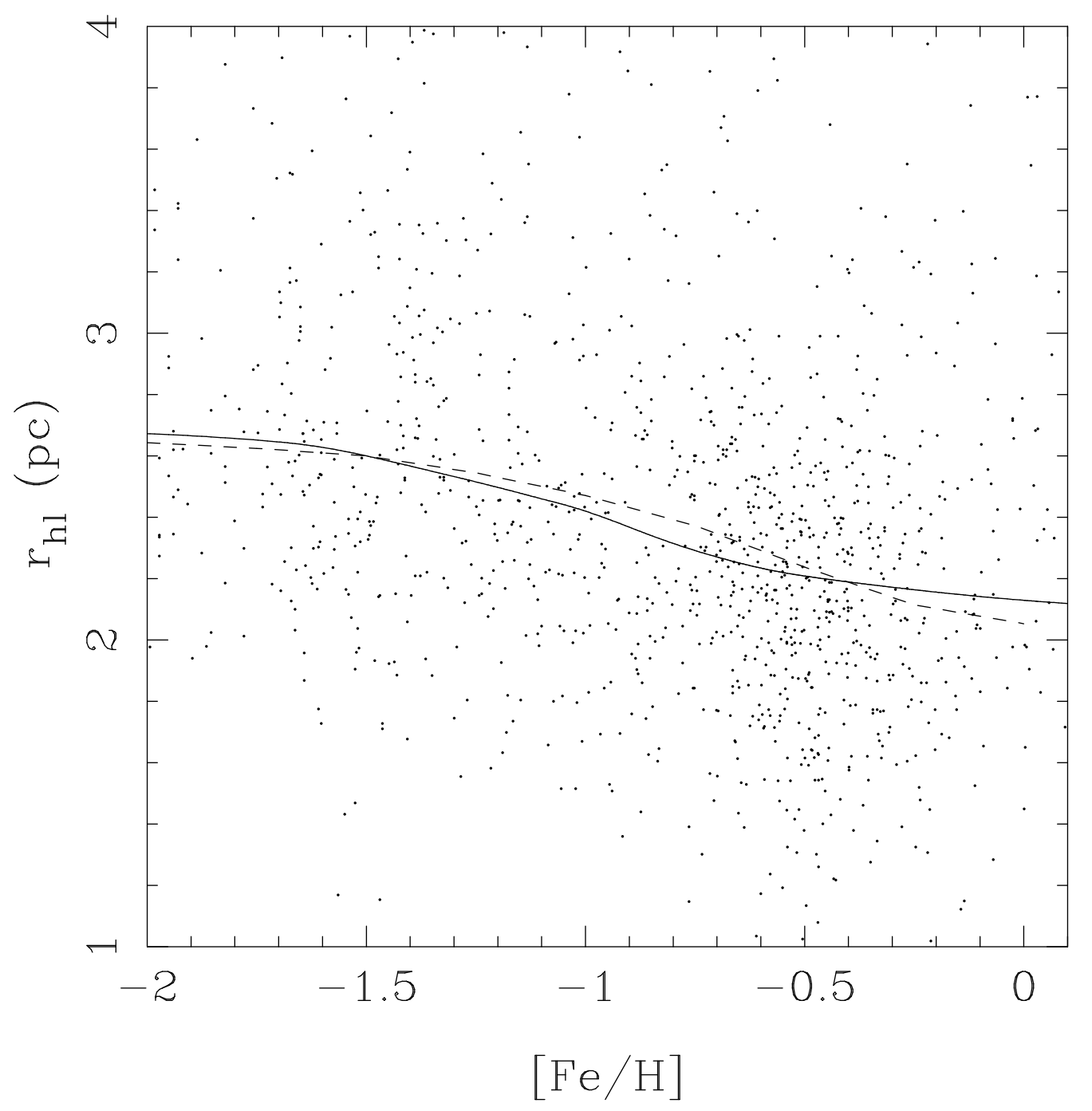

Figure 10: Half-light radii of GCs in M87 vs. [Fe/H] (derived from $g-z$ color). The overplotted solid and dashed lines are a running robust mean and a model fit (based upon King models; Jordán 2004) normalized to the metal-poor GCs. As is typical in massive galaxies, the metal-rich GCs are smaller than the metal-poor GCs (figure courtesy A. Jordán). 


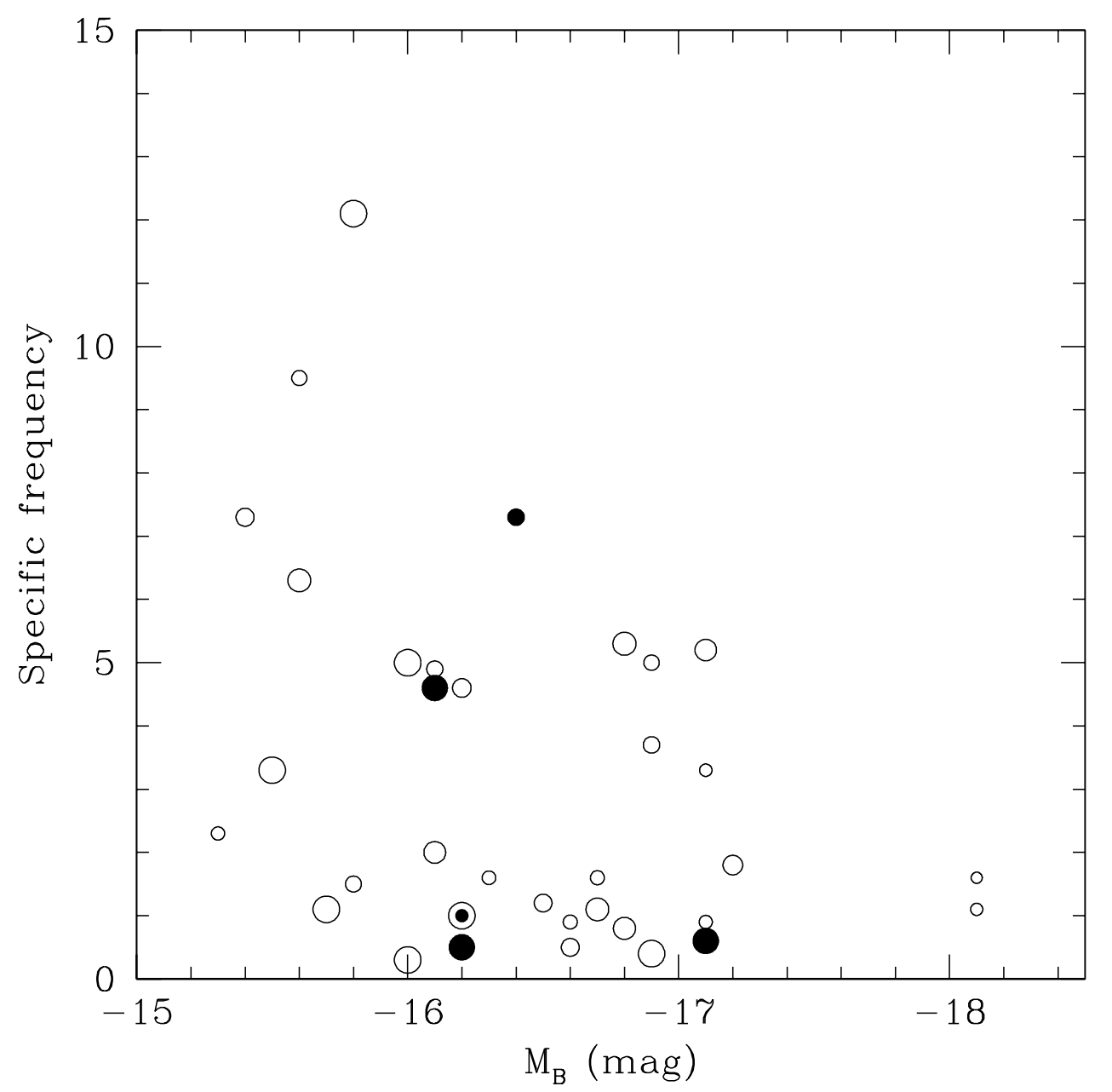

Figure 11: Specific frequency $\left(S_{N}\right)$ of dEs (open circles) and faint power-law Es (filled circles) vs. parent galaxy $M_{B}$. The size of the points is proportional to the fraction of blue GCs. There is only a weak trend of increasing $S_{N}$ with decreasing $M_{B}$, and no substantial difference between the two galaxy classes. However, there is some evidence for a bimodal distribution of $S_{N}$ (Strader et al. 2006). 


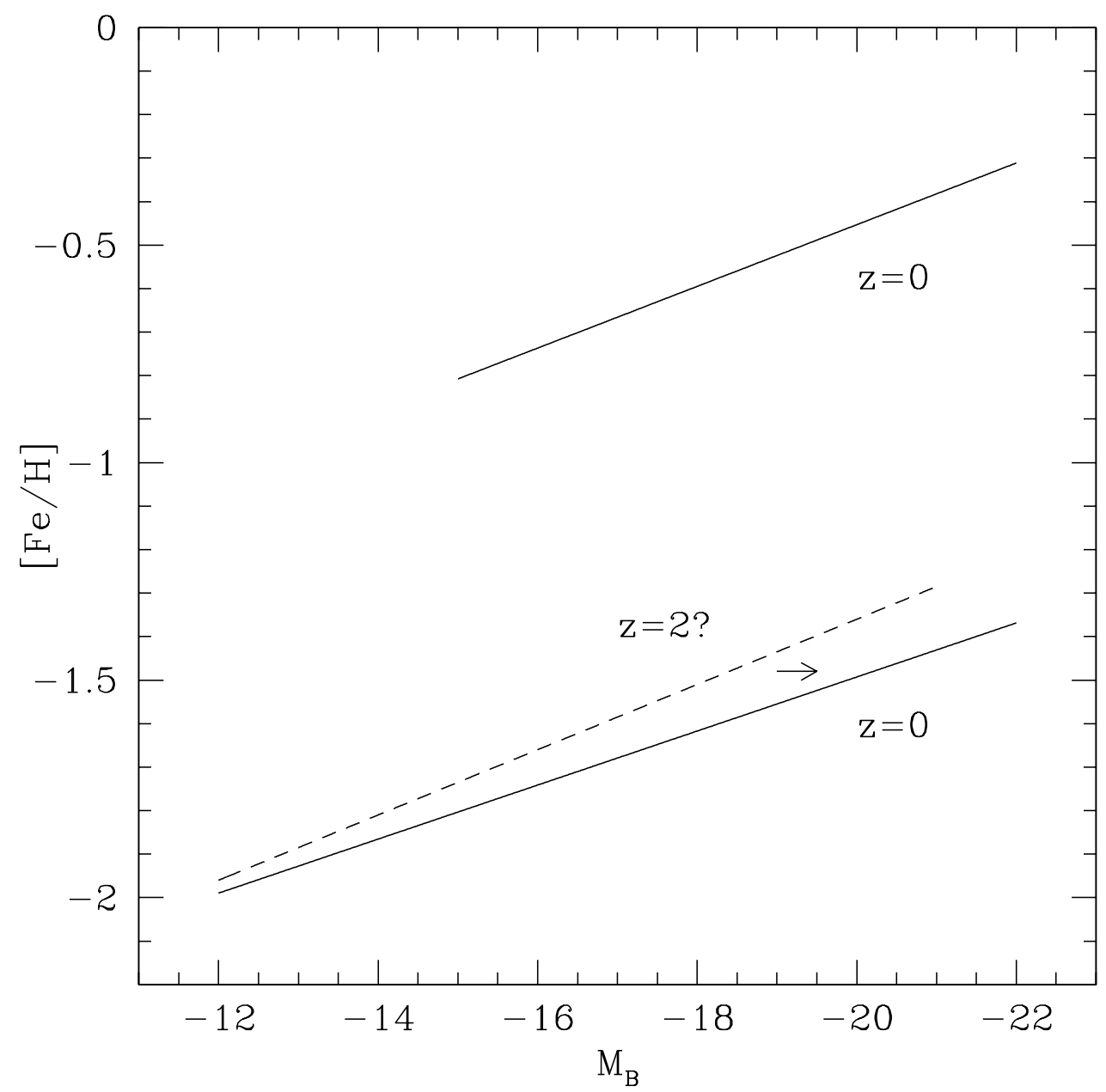

Figure 12: A schematic plot of the evolution of the metal-poor GC metallicitygalaxy luminosity relation due to biased galaxy merging. The solid lines show the $z=0$ relations for both subpopulations; the dashed line shows a conceptual metal-poor relation at higher redshift. 


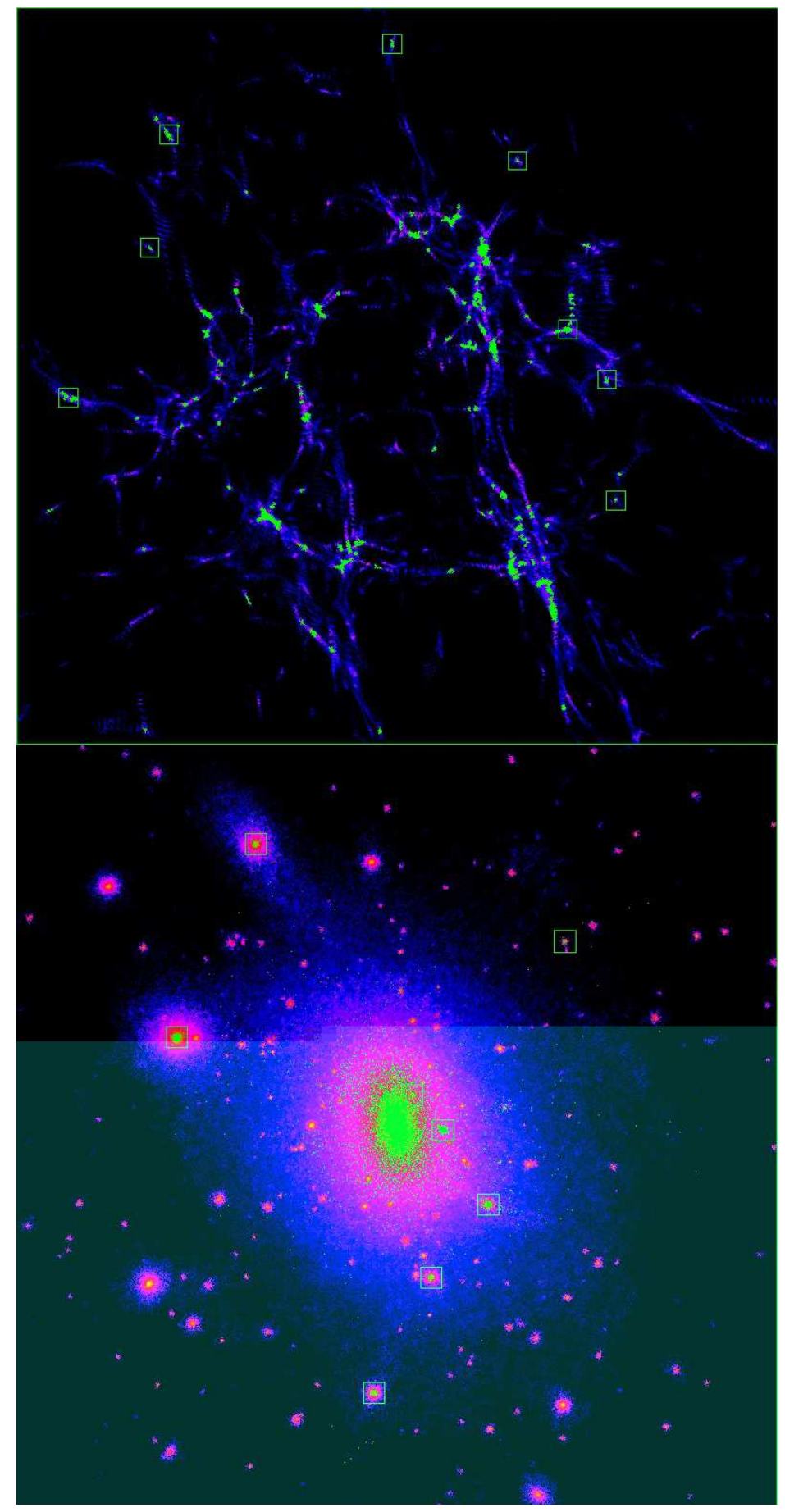

Figure 13: Two snapshots of a high-resolution dark matter simulation of the formation of $\mathrm{a} \sim 10^{12} M_{\odot}$ galaxy (Moore et al. 2006; Diemand et al. 2005). The top panel represents the simulation at $z=12$, and the bottom panel represents the present day. The blue to pink colors indicate dark matter of increasing density, while the green regions are those at $z=12$ with virial temperatures $>10^{4} \mathrm{~K}$ (such that atomic line cooling is effective, and gas can cool to form stars). At $z=12$, these green regions represent halos with masses $10^{8}-10^{10} M_{\odot}$, and these same green particles are marked in the $z=0$ snapshot. These high- $\sigma$ peaks collapse in a filamentary structure at high $z$ but are concentrated toward the center of the final galaxy. The boxes can be identified as dwarf satellites of the final galaxy. 


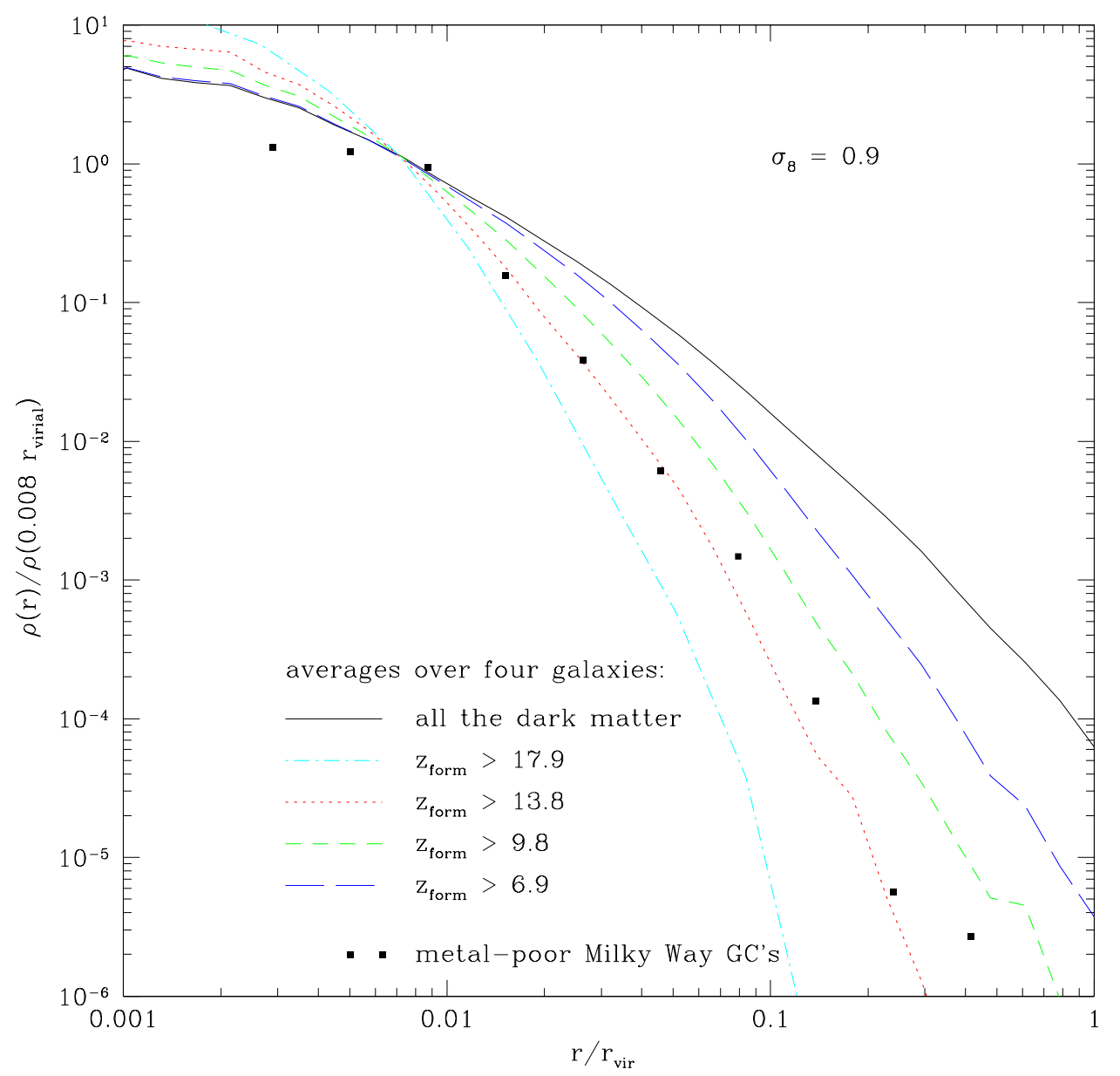

Figure 14: The radial distribution of metal-poor GCs in the Galaxy compared to results from numerical simulations of the formation of a Galaxy-like dark matter halo in a $\Lambda \mathrm{CDM}$ cosmology (Moore et al. 2006). The lines represent the radial distribution of $2.2 \times 10^{8} M_{\odot}$ mass halos that collapsed before: $z=17.9$ (dotdashed), $z=13.8$ (dotted), $z=9.8$ (short dashed), $z=6.9$ (long dashed), and the cumulative distribution (solid). The higher the redshift of collapse, the rarer the peak. Under the assumption that reionization truncates star formation in such halos, the comparison suggests reionization at $z \sim 12$. This redshift and mass combination corresponds to $\sim 2.5 \sigma$ peaks. 


\section{References}

Abadi M, Navarro J, Steinmetz M. 2006. astro-ph/0506659

Armandroff TE, Zinn R. 1988. Astron. J. 96:92

Ashman KM. 1990. Mon. Not. R. Astron. Soc. 247:662

Ashman KM, Bird CM. 1993. Astron. J. 106:2281

Ashman KM, Conti A, Zepf SE. 1995. Astron. J. 110:1164

Ashman KM, Zepf SE. 1998. Globular Cluster Systems. New York: Cambridge University Press

Ashman KM, Zepf SE. 1992. Ap. J. 384:50

Ashman KM, Zepf SE. 2001. Astron. J. 122:1888

Ashman KM, Walker S, Zepf SE. 2006. in preparation

Barmby P, Huchra JP, Brodie JP, Forbes DA, Schroder LL, Grillmair CJ. 2000. Astron. J. 119:727

Barmby P, Huchra JP. 2001. Astron. J. 122:2458

Bassino L, Richtler T, Dirsch B. 2006. Mon. Not. R. Astron. Soc. in press

Baumgardt H, Makino J. 2003. Mon. Not. R. Astron. Soc. 340:227

Beasley MA, Baugh CM, Forbes DA, Sharples RM, Frenk CS. 2002, Mon. Not. R. Astron. Soc. 333:383

Beasley MA, Baugh CM, Forbes DA, Sharples RM, Frenk CS. 2002. Mon. Not. R. Astron. Soc. 333:383

Beasley MA, Brodie JP, Strader J, Forbes DA, Proctor RN, et al. 2004. Astron. J. $128: 1623$

Beasley MA, Brodie JP, Strader J, Forbes DA, Proctor RN, et al. 2005. Astron. J. 129:1412

Bekki K, Beasley MA, Brodie JP, Forbes DA. 2005. Mon. Not. R. Astron. Soc. 363:1211

Bekki K, Couch WJ, Drinkwater MJ, Shioya Y. 2003. Mon. Not. R. Astron. Soc. 344:399

Bekki K, Forbes DA, Beasley MA, Couch WJ. 2002. Mon. Not. R. Astron. Soc. 335:1176

Bekki K, Forbes DA, Beasley MA, Couch WJ. 2003. Mon. Not. R. Astron. Soc. 344:1334

Bekki K, Forbes DA. 2005. Astron. Astrophys. in press

Bekki K, Harris WE, Harris GLH. 2003. Mon. Not. R. Astron. Soc. 338:587

Bell EF, et al. 2004. Ap. J. 608:752

Bernstein R, McWilliam A. 2005, astro-ph/0507042

Blakeslee JP, Tonry JL, Metzger MR. 1997. Astron. J. 114:482

Brodie JP, Hanes DA. 1986. Ap. J. 300:258

Brodie JP, Huchra JP. 1990. Ap. J. 362:503

Brodie JP, Huchra JP. 1991. Ap. J. 379:157

Brodie JP, Larsen SS, Kissler-Patig M. 2000. Ap. J. Lett. 543:19

Brodie JP, Larsen SS. 2002. Astron. J. 124:1410

Brodie JP, Schroder LL, Huchra JP, Phillips AC, Kissler-Patig M, Forbes DA. 1998. Astron. J. 116:691

Brodie JP, Strader J, Denicol G, Beasley MA, Cenarro AJ, et al. 2005. Astron. J. 129:2643

Brodie JP. 1981. Ph.D. Thesis

Brown TM, Ferguson HC, Smith E, Kimble RA, Sweigart AV, Renzini A, Rich RM, VandenBerg DA. 2003. Ap. J. Lett. 592:17 
Brown TM, Ferguson HC, Smith E, Kimble RA, Sweigart AV, Renzini A, Rich RM, VandenBerg DA. 2004. Ap. J. Lett. 613:125

Bruzual G, Charlot S. 2003. Mon. Not. R. Astron. Soc. 344:1000

Burgarella D, Kissler-Patig M, Buat V. 2001. Astron. J. 121:2647

Burkert A, Smith GH. 2000. Ap. J. 542:95

Burkert A, Brodie J, Larsen S. 2005. Ap. J. 628:231

Burstein D, Faber SM, Gaskell CM, Krumm N. 1984. Ap. J. 287:586

Burstein D, Heiles C. 1984. Ap. J. Suppl. 54:33

Burstein D, Li Y, Freeman KC, Norris JE, Bessell MS, et al. 2004. Ap. J. 614:158

Burstein D. 1987. In Nearly Normal Galaxies: From the Planck Time to the Present; Proceedings of the Eighth Santa Cruz Summer Workshop in Astronomy and Astrophysics, ed. S Faber, p. 47 New York: Springer-Verlag

Carney BW, Latham DW, Laird JB. 1990. Astron. J. 99:572

Carney BW. 1996. Publ. Astron. Soc. Pac. 108:900

Carretta E, Gratton RG. 1997. Astron. Ap. Suppl. 121:95

Cenarro AJ, et al. 2006. in preparation

Chaboyer B, Demarque P, Sarajedini A. 1996. Ap. J. 459:558

Chandar R, Bianchi L, Ford HC, Sarajedini A. 2002. Ap. J. 564:712

Chandar R, Whitmore B, Lee MG. 2004. Ap. J. 611:220

Cohen JG, Blakeslee JP, Ryzhov A. 1998. Ap. J. 496:808

Cohen JG, Blakeslee JP, Ct P. 2003. Ap. J. 592:866

Cohen JG, Blakeslee JP, Ryzhov A. 1998. Ap. J. 496:808

Cohen JG, Blakeslee JP. 1998. Astron. J. 115:2356

Cohen JG, Matthews K, Cameron PB. 2005. Ap. J. Lett. 634:45

Couture J, Harris WE, Allwright JWB. 1991. Ap. J. 372:97

Cowie LL, Songaila A, Hu EM, Cohen JG. 1996. Astron. J. 112:839

Côté P, Blakeslee JP, Ferrarese L, Jordán A, Mei S, et al. 2004. Ap. J. Suppl. 153:223

Côté P, Marzke RO, West MJ, Minniti D. 2000. Ap. J. 533:869

Côté P, Marzke RO, West MJ. 1998. Ap. J. 501:554

Côté P, McLaughlin DE, Cohen JG, Blakeslee JP. 2003. Ap. J. 591:850

Côté P, McLaughlin DE, Hanes DA, Bridges TJ, Geisler D, et al. 2001. Ap. J. 559:828

Côté P, West MJ, Marzke RO. 2002. Ap. J. 567:853

Côté P. 1999. Astron. J. 118:406

Davies RL, et al. 2001. Ap. J. Lett. 548:33

Dekel A, Birnboim Y. 2006. astro-ph/0412300

Dekel A, Stoehr F, Mamon GA, Cox TJ, Novak GS, Primack JR. 2005. Nature 437:707

Diemand J, Madau P, Moore B. 2005. Mon. Not. R. Astron. Soc. 364:367

Dirsch B, Richtler T, Bassino LP. 2003. Astron. Astrophys. 408:929

Dirsch B, Richtler T, Geisler D, Forte JC, Bassino LP, Gieren WP. 2003. Astron. J. 125:1908

Dirsch B, Schuberth Y, Richtler T. 2005. Astron. Astrophys. 433:43

Dressler A, Oemler A Jr, Couch WJ, Smail I, Ellis RS, et al. 1997. Ap. J. 490:577

Durrell PR, Harris WE, Pritchet CJ. 2001. Astron. J. 121:2557

Durrell PR, Harris WE, Geisler D, Pudritz RE. 1996. Astron. J. 112:972

Durrell PR, Harris WE, Pritchet CJ. 1994. Astron. J. 108:2114 
Durrell PR, McLaughlin DE, Harris WE, Hanes DA. 1996. Ap. J. 463:543

Elmegreen BG, Efremov YN. 1997. Ap. J. 480, 235

Evans NW, Wilkinson MI. 2000. Mon. Not. R. Astron. Soc. 316:929

Faber SM, et al. 2005, astro-ph/0506044

Faber SM, Tremaine S, Ajhar EA, Byun Y-I, Dressler A, et al. 1997. Astron. J. 114:1771

Fall SM, Rees MJ. 1985. Ap. J. 298:18

Fall SM, Rees MJ. 1988, IAU Symp126: The Harlow-Shapley Symposium on Globular Cluster Systems in Galaxies, 126:323

Fall SM, Zhang Q. 2001. Ap. J. 561:751

Forbes DA, Beasley MA, Brodie JP, Kissler-Patig M. 2001. Ap. J. Lett. 563:143

Forbes DA, Brodie JP, Grillmair CJ. 1997. Astron. J. 113:1652

Forbes DA, Brodie JP, Huchra J. 1996. Astron. J. 112:2448

Forbes DA, Brodie JP, Larsen SS. 2001. Ap. J. Lett. 556:83

Forbes DA, Forte JC. 2001. Mon. Not. R. Astron. Soc. 322:257

Forbes DA, Franx M, Illingworth GD, Carollo CM. 1996. Ap. J. 467:126

Forbes DA, Georgakakis AE, Brodie JP. 2001. Mon. Not. R. Astron. Soc. 325:1431

Forbes DA, Masters KL, Minniti D, Barmby P. 2000. Astron. Astrophys. 358:471

Forbes DA, Strader J, Brodie JP. 2004. Astron. J. 127:3394

Forbes DA. 2005, astro-ph/0511291

Forte JC, Faifer F, Geisler D. 2005. Mon. Not. R. Astron. Soc. 357:56

Forte JC, Geisler D, Ostrov PG, Piatti AE, Gieren W. 2001. Astron. J. 121:1992

Fukugita, M, Hogan CJ, Peebles PJE. 1998. Ap. J. 503:518

Fulbright J, McWilliam A, Rich M. 2006. astro-ph/0510408

Fusi Pecci F, Bellazzini M, Buzzoni A, De Simone E, Federici L, Galleti S. 2005.

Astron. J. 130:554

Gebhardt K, Kissler-Patig M. 1999. Astron. J. 118:1526

Geisler D, Lee MG, Kim E. 1996. Astron. J. 111:1529

Goerdt T, Moore B, Read JI, Stadel J, Zemp M. 2006, astro-ph/0601404

Gomez M, Richtler T. 2004. Astron. Astrophys. 415:499

Goudfrooij P, Alonso MV, Maraston C, Minniti D. 2001. Mon. Not. R. Astron.

Soc. 328:237

Goudfrooij P, Gilmore D, Whitmore BC, Schweizer F. 2004. Ap. J. Lett. 613:121

Goudfrooij P, Mack J, Kissler-Patig M, Meylan G, Minniti D. 2001. Mon. Not. R. Astron. Soc. 322:643

Goudfrooij P, Strader J, Brenneman L, Kissler-Patig M, Minniti D, Edwin Huizinga J. 2003. Mon. Not. R. Astron. Soc. 343:665

Gratton R, Sneden C, Carretta E. 2004. Annu. Rev. Astron. Astrophys. 42:385

Grebel EK, Dolphin AE, Guhathakurta P. 2000. Astron. Gesellschaft Abstract Ser. 17:79 (Abstr.)

Gregg MD. 1994. Astron. J. 108:2164

Guhathakurta P, Ostheimer JC, Gilbert KM, Rich RM, Majewski SR, et al. 2005. astro-ph/0502366

Hansen J, Moore B. 2006. astro-ph/0411473

Harris GLH, Geisler D, Harris HC, Hesser JE. 1992. Astron. J. 104:613 
Harris GLH, Harris WE, Geisler D. 2004. Astron. J. 128:723

Harris GLH, Harris WE, Poole GB. 1999. Astron. J. 117:855

Harris WE, Harris GLH, Holland ST, McLaughlin DE. 2002. Astron. J. 124:1435

Harris WE, Harris GLH. 2001. Astron. J. 122:3065

Harris WE, Harris GLH. 2002. Astron. J. 123:3108

Harris WE, Kavelaars JJ, Hanes DA, Hesser JE, Pritchet CJ. 2000. Ap. J. 533:137

Harris WE, Pudritz RE. 1994. Ap. J. 429:177

Harris WE, van den Bergh S. 1981. Astron. J. 86:1627

Harris WE. 1986. Astron. J. 91:822

Harris WE. 1991. Annu. Rev. Astron. Astrophys. 29:543

Harris WE. 2001. Saas-Fee Advanced Course 28: Star Clusters 28:223

Harris WE. 2003, Extragalactic Globular Cluster Systems, 317

Hempel M, Hilker M, Kissler-Patig M, Puzia TH, Minniti D, Goudfrooij P. 2003. Astron. Astrophys. 405:487

Hempel M, Kissler-Patig M. 2004. Astron. Astrophys. 419:863

Hempel M, Kissler-Patig M. 2004. Astron. Astrophys. 428:459

Hilker M, Infante L, Richtler T. 1999. Astron. Astrophy. Suppl. 138:55

Hilker M. 1998. Ph.D. Thesis

Hodge PW, Dolphin AE, Smith TR, Mateo M. 1999. Ap. J. 521:577

Holtzman JA, et al. 1992. Astron. J. 103:691

Huchra JP, Brodie JP, Kent SM. 1991. Ap. J. 370:495

Huxor AP, Tanvir NR, Irwin MJ, Ibata R, Collett JL, et al. 2005. Mon. Not. R. Astron. Soc. 360:1007

Hwang N, et al. 2005, IAU Colloq198: Near-fields cosmology with dwarf elliptical galaxies, 257

Hwang N, Lee MG. 2006, astro-ph/0601280

Jordán A. 2004. Ap. J. Lett. 613:117

Karachentsev ID, Karachentseva VE, Dolphin AE, Geisler D, Grebel EK, et al. 2000. Astron. Astrophys. 363:117

Karachentsev ID, Sharina ME, Grebel EK, Dolphin AE, Geisler D, et al. 2000. Ap. J. 542:128

Kent SM. 1992. Ap. J. 387:181

Kissler-Patig M, Ashman KM, Zepf SE, Freeman KC. 1999. Astron. J. 118:197

Kissler-Patig M, Forbes DA, Minniti D. 1998. Mon. Not. R. Astron. Soc. 298:1123

Kissler-Patig M. 2000. In Reviews in Modern Astronomy 13 : New Astrophysical Horizons, ed. RE Schielicke, p. 13. Hamburg, Germany: Astronomische Gesellschaft

Kormendy J. 1985. Ap. J. 295:73

Kormendy J. 1987. Nearly Normal Galaxies. From the Planck Time to the Present, 163

Kormendy J, Bender R. 1996. Ap. J. 464:119

Kormendy J, Kennicutt RC Jr. 2004. Annu. Rev. Astron. Astrophys. 42:603

Kormendy J, et al. 2006. in preparation

Korn AJ, Maraston C, Thomas D. 2005. Astron. Astrophys. 438:685

Kraft RP, Ivans II. 2003. Publ. Astron. Soc. Pac. 115:804

Kravtsov AV, Gnedin OY. 2005. Ap. J. 623:650

Kundu A, Whitmore BC, Sparks WB, Macchetto FD, Zepf SE, Ashman KM. 
1999. Ap. J. 513:733

Kundu A, Whitmore BC. 1998. Astron. J. 116:2841

Kundu A, Whitmore BC. 2001. Astron. J. 121:2950

Kundu A, Whitmore BC. 2001. Astron. J. 122:1251

Kundu A, et al. 2005. Ap. J. Lett. 634:41

Kuntschner H, Ziegler BL, Sharples RM, Worthey G, Fricke KJ. 2002. Astron. Astrophys. 395:761

Larsen SS, Brodie JP, Hunter DA. 2004. Astron. J. 128:2295

Larsen SS, Brodie JP, Strader J. 2005. Astron. Astrophys. 443:413

Larsen SS, Brodie JP, Beasley MA, Forbes DA, Kissler-Patig M, et al. 2003. Ap. J. 585:767

Larsen SS, Brodie JP, Beasley MA, Forbes DA. 2002. Astron. J. 124:828

Larsen SS, Brodie JP, Huchra JP, Forbes DA, Grillmair CJ. 2001. Astron. J. 121:2974

Larsen SS, Brodie JP, Sarajedini A, Huchra JP. 2002. Astron. J. 124:2615

Larsen SS, Brodie JP. 2000. Astron. J. 120:2938

Larsen SS, Brodie JP. 2002. Astron. J. 123:1488

Larsen SS, Brodie JP. 2003. Ap. J. 593:340

Larsen SS, Forbes DA, Brodie JP. 2001. Mon. Not. R. Astron. Soc. 327:1116

Larsen SS, Richtler T. 2000. Astron. Astrophys. 354:836

Law DR, Johnston KV, Majewski SR. 2005. Ap. J. 619:807

Layden AC, Sarajedini A. 2000. Astron. J. 119:1760

Lynden-Bell D. 1975. Vistas in Astronomy 19:299

Majewski SR, Patterson RJ, Dinescu DI, Johnson WY, Ostheimer JC, et al. 2000. In Proceedings of the 35th Liege International Astrophysics Colloquium : The Galactic Halo; From Globular Cluster to Field Stars, eds. A Noels, P Magain, D Caro, E Jehin, G Parmentier, AA Thoul, pp. 61926. Liege, Belgium: Institut d'Astrophysique et de Geophysique

Maraston C, Bastian N, Saglia RP, Kissler-Patig M, Schweizer F, Goudfrooij P. 2004. Astron. Astrophys. 416:467

Maraston C. 2005. Mon. Not. R. Astron. Soc. 362:799

Martin NF, Ibata RA, Bellazzini M, Irwin MJ, Lewis GF, Dehnen W. 2004.

Mon. Not. R. Astron. Soc. 348:12

Mashchenko S, Sills A. 2004. Ap. J. Lett. 605:121

Mashchenko S, Sills A. 2005. Ap. J. 619:243 (2005a)

Mashchenko S, Sills A. 2005. Ap. J. 619:258 (2005b)

Mashchenko S, Couchman H, Sills A. astro-ph/0511361

Matteucci F. 1994. Astron. Astrophys. 288:57

McCrady N, Gilbert AM, Graham JR. 2003. Ap. J. 596:240

McCrady N, Graham JR, Vacca WD. 2005. Ap. J. 621:278

McLaughlin DE. 1994. Publ. Astron. Soc. Pac. 106:47

McLaughlin DE. 1999. Astron. J. 117:2398

Meylan G, Heggie DC. 1997. Astron. Astrophys. Rev. 8:1

Mighell KJ, Sarajedini A, French RS. 1998. Astron. J. 116:2395

Miller BW, Lotz JM, Ferguson HC, Stiavelli M, Whitmore BC. 1998. Ap. J. Lett. 508:133

Miller BW, Whitmore BC, Schweizer F, Fall SM. 1997. Astron. J. 114:2381

Minniti D, Meylan G, Kissler-Patig M. 1996. Astron. Astrophys. 312:49

Minniti D. 1995. Astron. J. 109:1663

Moore B, Katz N, Lake G, Dressler A, Oemler A Jr. 1996. Nature 379:613 
Moore B, Lake G, Katz N. 1998. Ap. J. 495:139

Moore B. 1996. Ap. J. Lett. 461:13

Moore, B. et al. 2006. astro-ph/0510370

Morrison HL, Harding P, Perrett K, Hurley-Keller D. 2004. Ap. J. 603:87

Mould J, Kristian J. 1986. Ap. J. 305:591

Muzzio JC. 1987. Publ. Astron. Soc. Pac. 99:245

Olsen KAG, Miller BW, Suntzeff NB, Schommer RA, Bright J. 2004. Astron. J. 127:2674

Ostrov P, Geisler D, Forte JC. 1993. Astron. J. 105:1762

Ostrov PG, Forte JC, Geisler D. 1998. Astron. J. 116:2854

Peng EW, Ford HC, Freeman KC. 2004. Ap. J. 602:685

Peng EW, Ford HC, Freeman KC. 2004. Ap. J. 602:705

Peng EW, Jordán A, Ct P, Blakeslee JP, Ferrarese L, et al. 2006. astro-ph/0509654

Peng EW, et al. 2006. astro-ph/0511251

Perrett KM, Bridges TJ, Hanes DA, Irwin MJ, Brodie JP, et al. 2002. Astron. J. 123:249

Perrett KM, Stiff DA, Hanes DA, Bridges TJ. 2003. Ap. J. 589:790

Phillipps S, Drinkwater MJ, Gregg MD, Jones JB. 2001. Ap. J. 560:201

Pierce M, Brodie JP, Forbes DA, Beasley MA, Proctor R, Strader J. 2005.

Mon. Not. R. Astron. Soc. 358:419

Proctor RN, Forbes DA, Beasley MA. 2004. Mon. Not. R. Astron. Soc. 355:1327

Proctor RN, Sansom AE. 2002. Mon. Not. R. Astron. Soc. 333:517

Puzia TH, Kissler-Patig M, Brodie JP, Huchra JP. 1999. Astron. J. 118:2734

Puzia TH, Kissler-Patig M, Brodie JP, Schroder LL. 2000. Astron. J. 120:777

Puzia TH. 2003, Ph.D. Thesis

Puzia TH, Kissler-Patig M, Thomas D, Maraston C, Saglia RP, et al. 2004. Astron. Astrophys. 415:123

Puzia TH, Kissler-Patig M, Thomas D, Maraston C, Saglia RP, et al. 2005.

Astron. Astrophys. 439:997

Puzia TH, Perrett KM, Bridges TJ. 2005. Astron. Astrophys. 434:909

Puzia TH, Saglia RP, Kissler-Patig M, Maraston C, Greggio L, et al. 2002.

Astron. Astrophys. 395:45

Puzia TH, Zepf SE, Kissler-Patig M, Hilker M, Minniti D, Goudfrooij P. 2002.

Astron. Astrophys. 391:453

Read JI, Wilkinson MI, Evans NW, Gilmore G, Kleyna JT. 2006. astro-ph/0511759

Rejkuba M, Greggio L, Harris WE, Harris GLH, Peng EW. 2005. Ap. J. 631:262

Rejkuba M, Minniti D, Silva DR, Bedding TR. 2003. Astron. Astrophys. 411:351

Rhode KL, Zepf SE, Santos MR. 2005. Ap. J. Lett. 630:21

Rhode KL, Zepf SE. 2001. Astron. J. 121:210

Rhode KL, Zepf SE. 2003. Astron. J. 126:2307

Rhode KL, Zepf SE. 2004. Astron. J. 127:302

Rich RM, Corsi CE, Cacciari C, Federici L, Fusi Pecci F, Djorgovski SG, Freedman WL. 2005. Astron. J. 129:2670

Richtler T. 2006. astro-ph/0512545

Richtler T, Dirsch B, Gebhardt K, Geisler D, Hilker M, et al. 2004. Astron. J. 127:2094

Romanowsky AJ, Douglas NG, Arnaboldi M, Kuijken K, Merrifield MR, et al. 
2003. Science 301:1696

Rose JA. 1985. Astron. J. 90:1927

Sadler EM, Rich RM, Terndrup DM. 1996. Astron. J. 112:171

Saitoh et al. 2005. Astron. J. in press

Santos MR. 2003. Extragalactic Globular Cluster Systems, 348

Sarajedini A, Geisler D, Schommer R, Harding P. 2000. Astron. J. 120:2437

Sarajedini A, Jablonka P. 2005. Astron. J. 130:1627

Schiavon R. 2006. ApJS, submitted

Schiavon RP, Faber SM, Castilho BV, Rose JA. 2002. Ap. J. 580:850

Schiavon RP, Rose JA, Courteau S, MacArthur LA. 2004. Ap. J. Lett. 608:33

Schroder LL, Brodie JP, Kissler-Patig M, Huchra JP, Phillips AC. 2002. Astron. J. 123:2473

Schuberth, et al. 2006. in preparation

Schommer RA, Olszewski EW, Suntzeff NB, Harris HC. 1992. Astron. J. 103:447

Schweizer F. 1987. In Nearly Normal Galaxies: From the Planck Time to the Present; Proceedings of the Eighth Santa Cruz Summer Workshop in Astronomy and Astrophysics, ed. S Faber, p. 18. New York: Springer-Verlag

Searle L, Wilkinson A, Bagnuolo WG. 1980. Ap. J. 239:803

Searle L, Zinn R. 1978. Ap. J. 225:357

Secker J, Geisler D, McLaughlin DE, Harris WE. 1995. Astron. J. 109:1019

Shapley H. 1939. Proc. Nat. Acad. Sci. 25:423

Shapley H. 1939. Proc. Nat. Acad. Sci. 25:565

Sharina ME, Sil'chenko OK, Burenkov AN. 2003. Astron. Astrophys. 397:831

Smith GH, Burkert A. 2002. Ap. J. Lett. 578:51

Smith LJ, Gallagher JS. 2001. Mon. Not. R. Astron. Soc. 326:1027

Sohn ST. et al. 2005. astro-ph/0510413

Soria R, Mould JR, Watson AM, Gallagher JS III, Ballester GE, et al. 1996. Ap. J. 465:79

Spitzer L. 1987. Dynamical Evolution of Globular Clusters. Princeton, NJ: Princeton University Press

Stetson PB, Vandenberg DA, Bolte M. 1996. Publ. Astron. Soc. Pac. 108:560

Strader J, Brodie JP, Schweizer F, Larsen SS, Seitzer P. 2003. Astron. J. 125:626

Strader J, Brodie JP, Forbes DA, Beasley MA, Huchra JP. 2003. Astron. J. 125:1291

Strader J, Brodie JP, Forbes DA. 2004. Astron. J. 127:295

Strader J, Brodie JP, Forbes DA. 2004. Astron. J. 127:3431

Strader J, Brodie JP. 2004. Astron. J. 128:1671

Strader J, Brodie JP, Cenarro AJ, Beasley MA, Forbes DA. 2005. Astron. J. 130:1315

Strader J, Brodie J, Beasley M, Spitler L. 2006. astro-ph/0508001

Tantalo R, Chiosi C, Bressan A. 1998. Astron. Astrophys. 333:419

Thomas D, Maraston C, Bender R. 2003. Mon. Not. R. Astron. Soc. 339:897

Thomas D, Maraston C, Bender R, de Oliveira CM. 2005, Ap. J. 621:673

Toomre A, Toomre J. 1972. Ap. J. 178:623

Toomre A. 1977. In Evolution of Galaxies and Stellar Populations, Proceedings of a Conference at Yale University, eds. BM Tinsley, RB Larson, p. 40. New Haven: Yale University Observatory

Trager SC, Faber SM, Worthey G, Gonzlez JJ. 2000. Astron. J. 120:165 
Trager SC, Worthey G, Faber SM, Burstein D, Gonzalez JJ. 1998. Ap. J. Suppl. 116:1

Trager SC. 2004. Origin and Evolution of the Elements 391

Tripicco MJ, Bell RA. 1995. Astron. J. 110:3035

van den Bergh S, Mackey AD. 2004. Mon. Not. R. Astron. Soc. 354:713

van den Bergh S, Morbey C, Pazder J. 1991. Ap. J. 375:594

van den Bergh S. 1975. Annu. Rev. Astron. Astrophys. 13:217

van den Bergh S. 1982. Publ. Astron. Soc. Pac. 94:459

van den Bergh S. 1994, In: Smith R.C., Storm J. (eds.) The Local Group. ESO: Garching

van den Bergh S. 1999. Astron. Astrophys. Rev. 9:273

van den Bergh S. 2004. Astron. J. 127:897

Vesperini E, Heggie DC. 1997. Mon. Not. R. Astron. Soc. 289:898

Vesperini E, Zepf SE, Kundu A, Ashman KM. 2003. Ap. J. 593:760

Vesperini E, Zepf SE. 2003. Ap. J. Lett. 587:97

Vesperini E. 2000. Mon. Not. R. Astron. Soc. 318:841

Vesperini E. 2001. Mon. Not. R. Astron. Soc. 322:247

Walcher CJ, Fried JW, Burkert A, Klessen RS. 2003. Astron. Astrophys. 406:847

West MJ, Côté P, Marzke RO, Jordán A. 2004. Nature. 427:31

West MJ. 1993. Mon. Not. R. Astron. Soc. 265:755

Whitmore BC, Schweizer F, Kundu A, Miller BW. 2002. Astron. J. 124:147

Whitmore BC, Sparks WB, Lucas RA, Macchett FD, Biretta JA. 1995. Ap. J.

Lett. $454: 73$

Whitmore BC, Schweizer F. 1995. Astron. J. 109:960

Whitmore BC, Zhang Q. 2002. Astron. J. 124:1418

Williams BF, Hodge PW. 2001. Ap. J. 548:190

Wolf M. et al. 2006. Ap. J. submitted

Woodley KA, Harris WE, Harris GLH. 2005. Astron. J. 129:2654

Woodworth SC, Harris WE. 2000. Astron. J. 119:2699

Worthey G, Faber SM, Gonzlez JJ. 1992. Ap. J. 398:69

Worthey G, Faber SM, Gonzlez JJ, Burstein D. 1994. Ap. J. Suppl. 94:687

Worthey G, Espaa A, MacArthur LA, Courteau S. 2005. Ap. J. 631:820

Worthey G. 1994. Ap. J. Suppl. 95:107

Wu X, Tremaine S. 2006. astro-ph/0508463

Yoon S, Yi SK, Lee Y. 2006. astro-ph/0601526

Zepf SE, Ashman KM. 1993. Mon. Not. R. Astron. Soc. 264:611

Zepf SE, Beasley MA, Bridges TJ, Hanes DA, Sharples RM, et al. 2000. Astron. J. 120:2928

Zinn R, West MJ. 1984. Ap. J. Suppl. 55:45

Zinn R. 1985. Ap. J. 293:424 